Journal of Universal Mathematics

VoL.5 No.1 PP.1-23 (2022)

ISSN-2618-5660

DOI: $10.33773 /$ jum.974278

\title{
THEORY OF GENERALIZED SEPARATION AXIOMS IN GENERALIZED TOPOLOGICAL SPACES
}

\author{
M. I. KHODABOCUS AND N. UL-. H. SOOKIA
}

0000-0003-2252-4342 and 0000-0002-3155-0473

\begin{abstract}
In this paper, a new class of generalized separation axioms (briefly, $\mathfrak{g}-\mathfrak{T}_{\mathfrak{g}}$-separation axioms) whose elements are called $\mathfrak{g}-T_{\mathfrak{g}, K}, \mathfrak{g}-T_{\mathfrak{g}, F}, \mathfrak{g}-T_{\mathfrak{g}, H}$, $\mathfrak{g}-\mathrm{T}_{\mathfrak{g}, \mathrm{R}}, \mathfrak{g}-\mathrm{T}_{\mathfrak{g}, N}$-axioms is defined in terms of generalized sets (briefly, $\mathfrak{g}-\mathfrak{T}_{\mathfrak{g}}$-sets) in generalized topological spaces (briefly, $\mathcal{T}_{\mathfrak{g}}$-spaces) and the properties and characterizations of a $\mathcal{T}_{\mathfrak{g}}$-space endowed with each such $\mathfrak{g}-\mathrm{T}_{\mathfrak{g}, K}, \mathfrak{g}-\mathrm{T}_{\mathfrak{g}, F}, \mathfrak{g}-\mathrm{T}_{\mathfrak{g}, \mathrm{H}}$, $\mathfrak{g}-T_{\mathfrak{g}, R}, \mathfrak{g}-\mathrm{T}_{\mathfrak{g}, N}$-axioms are discussed. The study shows that $\mathfrak{g}-\mathrm{T}_{\mathfrak{g}, \mathrm{F}^{-}}$-axiom implies $\mathfrak{g}$ - $T_{\mathfrak{g}, K}$-axiom, $\mathfrak{g}$ - $T_{\mathfrak{g}, H}$-axiom implies $\mathfrak{g}$ - $T_{\mathfrak{g}, \mathrm{F}}$-axiom, $\mathfrak{g}$ - $\mathrm{T}_{\mathfrak{g}, \mathrm{R}}$-axiom implies

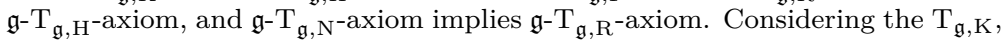

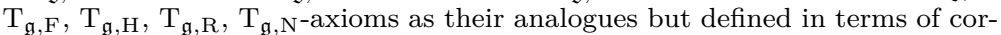
responding elements belonging to the class of open, closed, semi-open, semiclosed, preopen, preclosed, semi-preopen, and semi-preclosed sets, the study

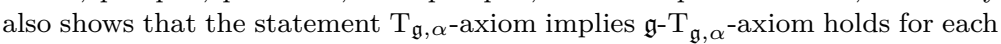
$\alpha \in\{\mathrm{K}, \mathrm{F}, \mathrm{H}, \mathrm{R}, \mathrm{N}\}$. Diagrams expose the various implications amongst the classes presented here and in the literature, and a nice application supports the overall theory.
\end{abstract}

\section{INTRODUCTION}

Whether it concerns the theory of $\mathcal{T}$-spaces or $\mathcal{T}_{\mathfrak{g}}$-spaces, the idea of adding a $\mathrm{T}_{\alpha}$ or a $\mathfrak{g}-\mathrm{T}_{\alpha}$-axiom ${ }^{1}($ with $\alpha=0,1,2, \ldots)$ to the axioms for a $\mathcal{T}$-space $\mathfrak{T}=(\Omega, \mathcal{T})$ to obtain a $\mathcal{T}^{(\alpha)}$-space $\mathfrak{T}^{(\alpha)}=\left(\Omega, \mathcal{T}^{(\alpha)}\right)$ or a $\mathfrak{g}$ - $\mathcal{T}^{(\alpha)}$-space $\mathfrak{g}$ - $\mathfrak{T}^{(\alpha)}=\left(\Omega, \mathfrak{g}-\mathcal{T}^{(\alpha)}\right)$ or, the idea of adding a $T_{\mathfrak{g}, \alpha}$ or a $\mathfrak{g}-\mathrm{T}_{\mathfrak{g}, \alpha}$-axiom (with $\alpha=0,1,2, \ldots$ ) to the axioms

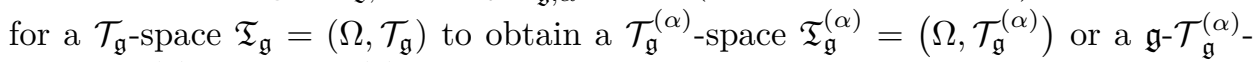
space $\mathfrak{g}-\mathfrak{T}_{\mathfrak{g}}^{(\alpha)}=\left(\Omega, \mathfrak{g}-\mathcal{T}_{\mathfrak{g}}^{(\alpha)}\right)$ has never played little role in Generalized Topology and Abstract Analysis [1, 2, 3]. Because the defining attributes of a $\mathcal{T}$-space in terms of a collection of $\mathcal{T}$-open or $\mathfrak{g}$ - $\mathcal{T}$-open sets or a $\mathcal{T}_{\mathfrak{g}}$-space in terms of a collection

Date: Received: 2021-07-25; Accepted: 2022-03-01.

2000 Mathematics Subject Classification. 54A05; 54D10, 54D15.

Key words and phrases. Generalized topological space, Generalized separation axioms, Generalized sets.

${ }^{1}$ Notes to the reader: The notations $\mathrm{T}_{\alpha}$-axiom and $\mathfrak{g}$ - $\mathrm{T}_{\alpha}$-axiom (with $\alpha=0,1,2, \ldots$ ), founded upon the notions of $\mathcal{T}$-open and $\mathfrak{g}$ - $\mathcal{T}$-open sets, respectively, designate an ordinary and a generalized separation axioms for a $\mathcal{T}$-space $\mathfrak{T}=(\Omega, \mathcal{T})$; the notations $\mathrm{T}_{\mathfrak{g}, \alpha}$-axiom and $\mathfrak{g}$ - $\mathrm{T}_{\mathfrak{g}, \alpha^{-}}$ axiom (with $\alpha=0,1,2, \ldots$ ), founded upon $\mathcal{T}_{\mathfrak{g}}$-open and $\mathfrak{g}$ - $\mathcal{T}_{\mathfrak{g}}$-open sets, respectively, designate an ordinary and a generalized separation axioms for a $\mathcal{T}_{\mathfrak{g}}$-space $\mathfrak{T}_{\mathfrak{g}}=\left(\Omega, \mathcal{T}_{\mathfrak{g}}\right)$. 
of $\mathcal{T}_{\mathfrak{g}}$-open or $\mathfrak{g}-\mathcal{T}_{\mathfrak{g}}$-open sets, respectively, does little to guarantee that the points in the $\mathcal{T}$-space $\mathfrak{T}$ or the $\mathcal{T}_{\mathfrak{g}}$-space $\mathfrak{T}_{\mathfrak{g}}$ are somehow distinct or far apart. The more types and categories of $\mathrm{T}_{\alpha}$ or $\mathfrak{g}-\mathrm{T}_{\alpha}$-axioms or $\mathfrak{g}$ - $\mathrm{T}_{\alpha}$ or $\mathfrak{g}$ - $\mathrm{T}_{\mathfrak{g}, \alpha}$-axioms (with $\alpha=0$, $1,2, \ldots)$ are added to the axioms for a $\mathcal{T}$-space or a $\mathcal{T}_{\mathfrak{g}}$-space, respectively, the greater the role they will play in any topological endeavours $[4,5,6,7,8]$. For instance, for a sequence $\left\langle\mathfrak{g}-\mathrm{T}_{\mathfrak{g}, \alpha}, \mathfrak{g}-\mathrm{T}_{\mathfrak{g}, \beta}, \mathfrak{g}-\mathrm{T}_{\mathfrak{g}, \gamma}\right\rangle$ (with $\alpha, \beta, \gamma=0,1,2, \ldots$ ), the $\mathfrak{g}-\mathrm{T}_{\mathfrak{g}, \alpha}, \mathfrak{g}-\mathrm{T}_{\mathfrak{g}, \beta}$ and $\mathfrak{g}-\mathrm{T}_{\mathfrak{g}, \gamma}$-axioms can be arranged in increasing order of strength in the sense that $\mathfrak{g}-\mathrm{T}_{\mathfrak{g}, \gamma}$ implies $\mathfrak{g}-\mathrm{T}_{\mathfrak{g}, \beta}$ and the latter implies $\mathfrak{g}-\mathrm{T}_{\mathfrak{g}, \alpha}$.

In the literature of $\mathcal{T}$-spaces and $\mathcal{T}_{\mathfrak{g}}$-spaces, respectively, several classes of $\mathrm{T}_{\alpha}$, $\mathfrak{g}$ - $\mathrm{T}_{\alpha}$-axioms, founded upon the concepts of $\mathcal{T}, \mathfrak{g}$ - $\mathcal{T}$-open sets, and $\mathrm{T}_{\mathfrak{g}, \alpha}, \mathfrak{g}$ - $\mathrm{T}_{\mathfrak{g}, \alpha^{-}}$ axioms (with $\alpha=0,1,2, \ldots$ ), founded upon the concepts of $\mathcal{T}_{\mathfrak{g}}, \mathfrak{g}-\mathcal{T}_{\mathfrak{g}}$-open sets, have been introduced and studied $[9,10,11,12,13,14,15,16]$. The $\mathrm{T}_{\alpha}$-axioms called $\mathrm{T}_{\text {Kolmogorov }}, \mathrm{T}_{\text {Frchet }}, \mathrm{T}_{\text {Hausdorff }}, \mathrm{T}_{\text {Regular }}$, and $\mathrm{T}_{\text {Normal-axioms (shortly, }} \mathrm{T}_{\mathrm{K}}$, $T_{F}, T_{H}, T_{R}$, and $T_{N}$ ), founded upon the concepts of $\mathcal{T}$-open, closed sets, are four classical examples, among others, which have gained extensive studies [17]. The $\mathfrak{g}$ - $\mathrm{T}_{\alpha}$-axioms called generalized $\mathrm{T}_{\alpha}, \mathrm{S}_{\beta}$-axioms (with $\alpha=0,1,2 ; \beta=1,2$ ), founded upon $\mathfrak{g}-\mathcal{T}$-open sets instead of $\mathcal{T}$-open sets, are five examples of generalized $\mathrm{T}_{\alpha}$-axioms which have been discussed in the paper of [18]; the $\mathfrak{g}$ - $\mathrm{T}_{\mathfrak{g}, \alpha}$-axioms called generalized $\mathrm{T}_{\frac{\alpha}{8}}$-axioms (with $\alpha=2,3,4$ ), founded upon $\mathfrak{g}-\mathcal{T}_{\mathfrak{g}}$-open sets instead of $\mathcal{T}_{\mathfrak{g}}$-open sets, are three examples of generalized $\mathrm{T}_{\mathfrak{g}, \alpha}$-axioms which have been introduced and studied by [19]. Several other classes of $\mathrm{T}_{\alpha}, \mathfrak{g}$ - $\mathrm{T}_{\alpha}$-axioms for $\mathcal{T}$ spaces and $\mathrm{T}_{\mathfrak{g}, \alpha}, \mathfrak{g}$ - $\mathrm{T}_{\mathfrak{g}, \alpha}$-axioms for $\mathcal{T}_{\mathfrak{g}}$-spaces (with $\alpha=0,1,2, \ldots$ ) have also been introduced and discussed in many papers [20,21, 15, 3, 22, 23].

In view of the above references, we remark that the quintuple sequence $\left\langle\mathrm{T}_{\alpha}\right\rangle_{\alpha \in \Lambda}$, where $\Lambda=\{\mathrm{K}, \mathrm{F}, \mathrm{H}, \mathrm{R}, \mathrm{N}\}$, is based on the notions of $\mathcal{T}$-open, closed sets. From this remark and the conclusion drawn by [18], it is no error to state that $\left\langle\mathfrak{g}-\mathrm{T}_{\alpha}\right\rangle_{\alpha \in \Lambda}$ are based on the notions of $\mathfrak{g}-\mathcal{T}$-open, closed sets; $\left\langle\mathrm{T}_{\mathfrak{g}, \alpha}\right\rangle_{\alpha \in \Lambda}$ on the notions of $\mathcal{T}_{\mathfrak{g}}$-open, closed sets, and $\left\langle\mathfrak{g}-T_{\mathfrak{g}, \alpha}\right\rangle_{\alpha \in \Lambda}$ on the notions of $\mathfrak{g}-\mathcal{T}_{\mathfrak{g}}$-open, closed sets. Thus, the idea of adding a quintuple sequence $\left\langle\mathfrak{g}-\mathrm{T}_{\mathfrak{g}, \alpha}\right\rangle_{\alpha \in \Lambda}$ of $\mathfrak{g}$-T $\mathrm{T}_{\mathfrak{g}, \alpha}$-axioms (with $\Lambda=\{\mathrm{K}, \mathrm{F}, \mathrm{H}, \mathrm{R}, \mathrm{N}\}$ ), founded upon a new class of $\mathfrak{g}-\mathcal{T}_{\mathfrak{g}}$-open, closed sets, to the axioms for a $\mathcal{T}_{\mathfrak{g}}$-space $\mathfrak{T}_{\mathfrak{g}}=\left(\Omega, \mathcal{T}_{\mathfrak{g}}\right)$ to obtain a corresponding sequence $\left\langle\mathfrak{g}-\mathfrak{T}_{\mathfrak{g}}^{(\alpha)}=\right.$ $\left.\left(\Omega, \mathfrak{g}-\mathcal{T}_{\mathfrak{g}}^{(\alpha)}\right)\right\rangle_{\alpha \in \Lambda}$ of $\mathfrak{g}-\mathcal{T}_{\mathfrak{g}}^{(\alpha)}$-spaces might be an interesting subject of inquiry.

Hitherto, the introduction of several types of $\mathrm{T}_{\alpha}$ and $\mathfrak{g}$ - $\mathrm{T}_{\alpha}$-axioms in $\mathcal{T}$-spaces and $T_{\mathfrak{g}, \alpha}$ and $\mathfrak{g}$ - $T_{\mathfrak{g}, \alpha}$-axioms (with $\alpha=0,1,2, \ldots$ ) in $\mathcal{T}_{\mathfrak{g}}$-spaces have contributed extensively to the geometrical specifications of $\mathcal{T}$-spaces and $\mathcal{T}_{\mathfrak{g}}$-spaces. However, despite these contributions not a single work has been devoted to the generalization of the sequence $\left\langle\mathrm{T}_{\alpha}\right\rangle_{\alpha \in \Lambda}$ in terms of the notions of $\mathfrak{g}$ - $\mathcal{T}$-open, closed sets. With this view in mind, the idea therefore suggests itself, of introducing the generalized versions of the Kolmogorov, Fréchet, Hausdorff, Regular and Normal separation axioms in terms of the notions of $\mathfrak{g}$ - $\mathcal{T}$-open, closed sets in a $\mathcal{T}_{\mathfrak{g}}$-space, adequate for the obtention of $\mathfrak{g}-\mathcal{T}_{\mathfrak{g}}$-spaces in this direction.

In this paper, we attempt to make a contribution to such a development by introducing a new theory, called Theory of $\mathfrak{g}-\mathfrak{T}_{\mathfrak{g}}$-Separation Axioms, in which it is presented the generalized version of the sequence $\left\langle\mathrm{T}_{\alpha}\right\rangle_{\alpha \in \Lambda}$ in terms of the notions of $\mathfrak{g}-\mathcal{T}$-open, closed sets, discussing the fundamental properties and giving 
characterizations of its elements, on this ground and with respect to existing works $[?, 14]$.

The paper is organised as follows: In SECT. 2, preliminary notions are described in SECT. 2.1 and the main results of the $\mathfrak{g}$ - $\mathrm{T}_{\mathfrak{g}, \alpha}$-axioms in a $\mathcal{T}_{\mathfrak{g}}$-space are reported in SECT. 3. In SECT. 4, the establishment of the various relationships between these $\mathfrak{g}$ - $\mathrm{T}_{\mathfrak{g}, \alpha}$-axioms are discussed in SECT. 4.1. To support the work, a nice application of the $\mathfrak{g}$ - $T_{\mathfrak{g}, \alpha}$-axioms in a $\mathcal{T}_{\mathfrak{g}}$-space is presented in SECT. 4.2. Finally, SECT. 5 provides concluding remarks and future directions of the $\mathfrak{g}$ - $T_{\mathfrak{g}, \alpha}$-axioms in a $\mathcal{T}_{\mathfrak{g}}$-space.

\section{THEORY}

2.1. Preliminaries. Though foreign terms are neatly defined in [30], we thought it necessary to recall some basic definitions and notations of most essential concepts presented in [30].

The set $\mathfrak{U}$ represents the universe of discourse, fixed within the framework of the theory of $\mathfrak{g}-\mathfrak{T}_{\mathfrak{g}}$-separation axioms and containing as elements all sets $(\mathcal{T}, \mathfrak{g}-\mathcal{T}$, $\mathfrak{T}, \mathfrak{g}$-T-sets; $\mathcal{T}_{\mathfrak{g}}, \mathfrak{g}$ - $\mathcal{T}_{\mathfrak{g}}, \mathfrak{T}_{\mathfrak{g}}, \mathfrak{g}$ - $\mathfrak{T}_{\mathfrak{g}}$-sets $)$ considered in this theory, and $I_{n}^{0} \stackrel{\text { def }}{=}\{\nu \in$ $\left.\mathbb{N}^{0}: \nu \leq n\right\}$; index sets $I_{\infty}^{0}, I_{n}^{*}, I_{\infty}^{*}$ are defined in an analogous way. Granted $\Omega \subset \mathfrak{U}, \mathcal{P}(\Omega) \stackrel{\text { def }}{=}\left\{\mathcal{O}_{\mathfrak{g}, \nu}: \mathcal{O}_{\mathfrak{g}, \nu} \subseteq \Omega\right\}$ denotes the family of all subsets $\mathcal{O}_{\mathfrak{g}, 1}$, $\mathcal{O}_{\mathfrak{g}, 2}, \ldots$, of $\Omega$. A one-valued map of the type $\mathcal{T}_{\mathfrak{g}}: \mathcal{P}(\Omega) \longrightarrow \mathcal{P}(\Omega)$ satisfying $\mathcal{T}_{\mathfrak{g}}(\emptyset)=\emptyset, \mathcal{T}_{\mathfrak{g}}\left(\mathcal{O}_{\mathfrak{g}}\right) \subseteq \mathcal{O}_{\mathfrak{g}}$, and $\mathcal{T}_{\mathfrak{g}}\left(\bigcup_{\nu \in I_{\infty}^{*}} \mathcal{O}_{\mathfrak{g}, \nu}\right)=\bigcup_{\nu \in I_{\infty}^{*}} \mathcal{T}_{\mathfrak{g}}\left(\mathcal{O}_{\mathfrak{g}, \nu}\right)$ is called a $\mathfrak{g}-$ topology on $\Omega$, and the structure $\mathfrak{T}_{\mathfrak{g}} \stackrel{\text { def }}{=}\left(\Omega, \mathcal{T}_{\mathfrak{g}}\right)$ is called a $\mathcal{T}_{\mathfrak{g}}$-space, on which a quintuple sequence $\left\langle\mathfrak{g}-\mathrm{T}_{\mathfrak{g}, \alpha}\right\rangle_{\alpha \in \Lambda}$ of $\mathfrak{g}$ - $\mathrm{T}_{\mathfrak{g}, \alpha}$-axioms (with $\Lambda=\{\mathrm{K}, \mathrm{F}, \mathrm{H}, \mathrm{R}, \mathrm{N}\}$ ) will be discussed $[24,25,26]$.

The operator cl $\mathrm{cl}_{\mathfrak{g}}: \mathcal{P}(\Omega) \longrightarrow \mathcal{P}(\Omega)$ carrying each $\mathfrak{T}_{\mathfrak{g}}$-set $\mathcal{S}_{\mathfrak{g}} \subset \mathfrak{T}_{\mathfrak{g}, \Omega}$ into its closure $\operatorname{cl}_{\mathfrak{g}}\left(\mathcal{S}_{\mathfrak{g}}\right)=\mathfrak{T}_{\mathfrak{g}}-\operatorname{int}_{\mathfrak{g}}\left(\mathfrak{T}_{\mathfrak{g}} \backslash \mathcal{S}_{\mathfrak{g}}\right) \subset \mathfrak{T}_{\mathfrak{g}}$ is termed a $\mathfrak{g}$-closure operator and the operator int $: \mathcal{P}(\Omega) \longrightarrow \mathcal{P}(\Omega)$ carrying each $\mathfrak{T}_{\mathfrak{g}}$-set $\mathcal{S}_{\mathfrak{g}} \subset \mathfrak{T}_{\mathfrak{g}}$ into its interior $\operatorname{int}_{\mathfrak{g}}\left(\mathcal{S}_{\mathfrak{g}}\right)=\mathfrak{T}_{\mathfrak{g}}-\mathrm{cl}_{\mathfrak{g}}\left(\mathfrak{T}_{\mathfrak{g}} \backslash \mathcal{S}_{\mathfrak{g}}\right) \subset \mathfrak{T}_{\mathfrak{g}}$ is called a $\mathfrak{g}$-interior operator. Let $\complement: \mathcal{P}(\Omega) \longrightarrow$ $\mathcal{P}(\Omega)$ denotes the absolute complement with respect to the underlying set $\Omega \subset \mathfrak{U}$, and let $\mathcal{S}_{\mathfrak{g}} \subset \mathfrak{T}_{\mathfrak{g}}$ be any $\mathfrak{T}_{\mathfrak{g}}$-set. The classes

$$
\begin{aligned}
\mathcal{T}_{\mathfrak{g}} & \stackrel{\text { def }}{=}\left\{\mathcal{O}_{\mathfrak{g}} \subset \mathfrak{T}_{\mathfrak{g}}: \mathcal{O}_{\mathfrak{g}} \in \mathcal{T}_{\mathfrak{g}}\right\}, \\
\neg \mathcal{T}_{\mathfrak{g}} & \stackrel{\text { def }}{=}\left\{\mathcal{K}_{\mathfrak{g}} \subset \mathfrak{T}_{\mathfrak{g}}: \complement_{\Lambda}\left(\mathcal{K}_{\mathfrak{g}}\right) \in \mathcal{T}_{\mathfrak{g}}\right\},
\end{aligned}
$$

respectively, denote the classes of all $\mathcal{T}_{\mathfrak{g}}$-open and $\mathcal{T}_{\mathfrak{g}}$-closed sets relative to the $\mathfrak{g}$-topology $\mathcal{T}_{\mathfrak{g}}$, and the classes

$$
\begin{aligned}
\mathrm{C}_{\mathcal{T}_{\mathfrak{g}}}^{\text {sub }}\left[\mathcal{S}_{\mathfrak{g}}\right] \stackrel{\text { def }}{=}\left\{\mathcal{O}_{\mathfrak{g}} \in \mathcal{T}_{\mathfrak{g}}: \mathcal{O}_{\mathfrak{g}} \subseteq \mathcal{S}_{\mathfrak{g}}\right\}, \\
\mathrm{C}_{\neg \mathcal{T}_{\mathfrak{g}}}^{\text {sup }},\left[\mathcal{S}_{\mathfrak{g}}\right] \stackrel{\text { def }}{=}\left\{\mathcal{K}_{\mathfrak{g}} \in \neg \mathcal{T}_{\mathfrak{g}}: \mathcal{K}_{\mathfrak{g}} \supseteq \mathcal{S}_{\mathfrak{g}}\right\},
\end{aligned}
$$

respectively, denote the classes of $\mathcal{T}_{\mathfrak{g}}$-open subsets and $\mathcal{T}_{\mathfrak{g}}$-closed supersets (complements of the $\mathcal{T}_{\mathfrak{g}}$-open subsets) of the $\mathfrak{T}_{\mathfrak{g}}$-set $\mathcal{S}_{\mathfrak{g}} \subset \mathfrak{T}_{\mathfrak{g}}$ relative to the $\mathfrak{g}$-topology $\mathcal{T}_{\mathfrak{g}}$. To this end, the $\mathfrak{g}$-closure and the $\mathfrak{g}$-interior of a $\mathfrak{T}_{\mathfrak{g}}$-set $\mathcal{S}_{\mathfrak{g}} \subset \mathfrak{T}_{\mathfrak{g}}$ in a $\mathcal{T}_{\mathfrak{g}}$-space [27] define themselves as

$$
\operatorname{int}_{\mathfrak{g}}\left(\mathcal{S}_{\mathfrak{g}}\right) \stackrel{\text { def }}{=} \bigcup_{\mathcal{O}_{\mathfrak{g}} \in \mathrm{C}_{\mathcal{T}_{\mathfrak{g}}}^{\text {sub }}\left[\mathcal{S}_{\mathfrak{g}}\right]} \mathcal{O}_{\mathfrak{g}}, \quad \operatorname{cl}_{\mathfrak{g}}\left(\mathcal{S}_{\mathfrak{g}}\right) \stackrel{\text { def }}{=} \bigcap_{\mathcal{K}_{\mathfrak{g}} \in \mathrm{C}_{\neg \mathcal{T}_{\mathfrak{g}}}^{\text {sup }}\left[\mathcal{S}_{\mathfrak{g}}\right]} \mathcal{K}_{\mathfrak{g}} .
$$


Throughout this paper, the composition operators $\operatorname{cl}_{\mathfrak{g}} \circ \operatorname{int}_{\mathfrak{g}}(\cdot)$, $\operatorname{int}_{\mathfrak{g}} \circ \mathrm{cl}_{\mathfrak{g}}(\cdot)$, and $\mathrm{cl}_{\mathfrak{g}} \circ \operatorname{int}_{\mathfrak{g}} \circ \mathrm{cl}_{\mathfrak{g}}(\cdot)$, respectively, stand for the functionals $\operatorname{cl}_{\mathfrak{g}}\left(\operatorname{int}_{\mathfrak{g}}(\cdot)\right)$, $\operatorname{int}_{\mathfrak{g}}\left(\mathrm{cl}_{\mathfrak{g}}(\cdot)\right)$, and $\operatorname{cl}_{\mathfrak{g}}\left(\operatorname{int}_{\mathfrak{g}}\left(\mathrm{cl}_{\mathfrak{g}}(\cdot)\right)\right)$; other composition operators are defined similarly. Furthermore, the backslash $\mathfrak{T}_{\mathfrak{g}} \backslash \mathcal{S}_{\mathfrak{g}}$ refers to the set-theoretic difference $\mathfrak{T}_{\mathfrak{g}}-\mathcal{S}_{\mathfrak{g}}$. The mapping op g $_{\mathfrak{g}} \mathcal{P}(\Omega) \longrightarrow \mathcal{P}(\Omega)$ is called a $\mathfrak{g}$-operation on $\mathcal{P}(\Omega)$ if the following statements hold:

$$
\begin{aligned}
& \forall \mathcal{S}_{\mathfrak{g}} \in \mathcal{P}(\Omega) \backslash\{\emptyset\}, \exists\left(\mathcal{O}_{\mathfrak{g}}, \mathcal{K}_{\mathfrak{g}}\right) \in \mathcal{T}_{\mathfrak{g}} \backslash\{\emptyset\} \times \neg \mathcal{T}_{\mathfrak{g}} \backslash\{\emptyset\}: \\
& \left(\operatorname{op}_{\mathfrak{g}}(\emptyset)=\emptyset\right) \vee\left(\neg \mathrm{op}_{\mathfrak{g}}(\emptyset)=\emptyset\right),\left(\mathcal{S}_{\mathfrak{g}} \subseteq \operatorname{op}_{\mathfrak{g}}\left(\mathcal{O}_{\mathfrak{g}}\right)\right) \vee\left(\mathcal{S}_{\mathfrak{g}} \supseteq \neg \mathrm{op}_{\mathfrak{g}}\left(\mathcal{K}_{\mathfrak{g}}\right)\right),
\end{aligned}
$$

where $\neg \mathrm{op}_{\mathfrak{g}}: \mathcal{P}(\Omega) \longrightarrow \mathcal{P}(\Omega)$ is called the "complementary $\mathfrak{g}$-operation" on $\mathcal{P}(\Omega)$ and, for all $\mathfrak{T}_{\mathfrak{g}}$-sets $\mathcal{S}_{\mathfrak{g}}, \mathcal{S}_{\mathfrak{g}, \nu}, \mathcal{S}_{\mathfrak{g}, \mu} \in \mathcal{P}(\Omega) \backslash\{\emptyset\}$, the following axioms are satisfied:

- Ax. I. $\left(\mathcal{S}_{\mathfrak{g}} \subseteq \operatorname{op}_{\mathfrak{g}}\left(\mathcal{O}_{\mathfrak{g}}\right)\right) \vee\left(\mathcal{S}_{\mathfrak{g}} \supseteq \neg \mathrm{op}_{\mathfrak{g}}\left(\mathcal{K}_{\mathfrak{g}}\right)\right)$,

- Ax. II. $\left(\operatorname{op}_{\mathfrak{g}}\left(\mathcal{S}_{\mathfrak{g}}\right) \subseteq \mathrm{op}_{\mathfrak{g}} \circ \mathrm{op}_{\mathfrak{g}}\left(\mathcal{O}_{\mathfrak{g}}\right)\right) \vee\left(\neg \mathrm{op}_{\mathfrak{g}}\left(\mathcal{S}_{\mathfrak{g}}\right) \supseteq \neg \mathrm{op}_{\mathfrak{g}} \circ \neg \mathrm{op}_{\mathfrak{g}}\left(\mathcal{K}_{\mathfrak{g}}\right)\right)$,

- Ax. III. $\left(\mathcal{S}_{\mathfrak{g}, \nu} \subseteq \mathcal{S}_{\mathfrak{g}, \mu} \longrightarrow \operatorname{op}_{\mathfrak{g}}\left(\mathcal{O}_{\mathfrak{g}, \nu}\right) \subseteq \operatorname{op}_{\mathfrak{g}}\left(\mathcal{O}_{\mathfrak{g}, \mu}\right)\right) \vee\left(\mathcal{S}_{\mathfrak{g}, \mu} \subseteq \mathcal{S}_{\mathfrak{g}, \nu} \longleftarrow\right.$ $\left.\neg \mathrm{op}_{\mathfrak{g}}\left(\mathcal{K}_{\mathfrak{g}, \mu}\right) \supseteq \neg \mathrm{op}_{\mathfrak{g}}\left(\mathcal{K}_{\mathfrak{g}, \nu}\right)\right)$,

- Ax. IV. $\left(\operatorname{op}_{\mathfrak{g}}\left(\bigcup_{\sigma=\nu, \mu} \mathcal{S}_{\mathfrak{g}, \sigma}\right) \subseteq \bigcup_{\sigma=\nu, \mu} \mathrm{op}_{\mathfrak{g}}\left(\mathcal{O}_{\mathfrak{g}, \sigma}\right)\right) \vee\left(\neg \mathrm{op}_{\mathfrak{g}}\left(\bigcup_{\sigma=\nu, \mu} \mathcal{S}_{\mathfrak{g}, \sigma}\right) \supseteq\right.$ $\left.\bigcup_{\sigma=\nu, \mu} \neg \operatorname{op}_{\mathfrak{g}}\left(\mathcal{K}_{\mathfrak{g}, \sigma}\right)\right)$,

for some $\mathcal{T}_{\mathfrak{g}}$-open sets $\mathcal{O}_{\mathfrak{g}}, \mathcal{O}_{\mathfrak{g}, \nu}, \mathcal{O}_{\mathfrak{g}, \mu} \in \mathcal{T}_{\mathfrak{g}} \backslash\{\emptyset\}$ and $\mathcal{T}_{\mathfrak{g}}$-closed sets $\mathcal{K}_{\mathfrak{g}}, \mathcal{K}_{\mathfrak{g}, \nu}$, $\mathcal{K}_{\mathfrak{g}, \mu} \in \neg \mathcal{T}_{\mathfrak{g}}[28,29]$. The class $\mathcal{L}_{\mathfrak{g}}[\Omega]=\mathcal{L}_{\mathfrak{g}}^{\omega}[\Omega] \times \mathcal{L}_{\mathfrak{g}}^{\kappa}[\Omega]$, where

$$
\mathcal{L}_{\mathfrak{g}}[\Omega] \stackrel{\text { def }}{=}\left\{\operatorname{op}_{\mathfrak{g}, \nu \mu}(\cdot)=\left(\mathrm{op}_{\mathfrak{g}, \nu}(\cdot), \neg \mathrm{op}_{\mathfrak{g}, \mu}(\cdot)\right):(\nu, \mu) \in I_{3}^{0} \times I_{3}^{0}\right\}
$$

in the $\mathcal{T}_{\mathfrak{g}}$-space $\mathfrak{T}_{\mathfrak{g}}$, stands for the class of all possible $\mathfrak{g}$-operators and their complementary $\mathfrak{g}$-operators in the $\mathcal{T}_{\mathfrak{g}}$-space $\mathfrak{T}_{\mathfrak{g}}$. Its elements are defined as:

$$
\begin{aligned}
\operatorname{op}_{\mathfrak{g}}(\cdot) & \in \mathcal{L}_{\mathfrak{g}}^{\omega}[\Omega] \stackrel{\text { def }}{=}\left\{\operatorname{op}_{\mathfrak{g}, 0}(\cdot), \operatorname{op}_{\mathfrak{g}, 1}(\cdot), \operatorname{op}_{\mathfrak{g}, 2}(\cdot), \operatorname{op}_{\mathfrak{g}, 3}(\cdot)\right\} \\
& =\left\{\operatorname{int}_{\mathfrak{g}}(\cdot), \operatorname{cl}_{\mathfrak{g}} \circ \operatorname{int}_{\mathfrak{g}}(\cdot), \operatorname{int}_{\mathfrak{g}} \circ \mathrm{cl}_{\mathfrak{g}}(\cdot), \mathrm{cl}_{\mathfrak{g}} \circ \operatorname{int}_{\mathfrak{g}} \circ \mathrm{cl}_{\mathfrak{g}}(\cdot)\right\} ; \\
\neg \mathrm{op}_{\mathfrak{g}}(\cdot) & \in \mathcal{L}_{\mathfrak{g}}^{\kappa}[\Omega] \stackrel{\text { def }}{=}\left\{\neg \operatorname{op}_{\mathfrak{g}, 0}(\cdot), \neg \operatorname{op}_{\mathfrak{g}, 1}(\cdot), \neg \mathrm{op}_{\mathfrak{g}, 2}(\cdot), \neg \mathrm{op}_{\mathfrak{g}, 3}(\cdot)\right\} \\
& =\left\{\mathrm{cl}_{\mathfrak{g}}(\cdot), \operatorname{int}_{\mathfrak{g}} \circ \mathrm{cl}_{\mathfrak{g}}(\cdot), \operatorname{cl}_{\mathfrak{g}} \circ \operatorname{int}_{\mathfrak{g}}(\cdot), \operatorname{int}_{\mathfrak{g}} \circ \mathrm{cl}_{\mathfrak{g}} \circ \operatorname{int}_{\mathfrak{g}}(\cdot)\right\} .
\end{aligned}
$$

A $\mathfrak{T}_{\mathfrak{g}}$-set $\mathcal{S}_{\mathfrak{g}} \subset \mathfrak{T}_{\mathfrak{g}}$ in a $\mathcal{T}_{\mathfrak{g}}$-space is called a $\mathfrak{g}$ - $\mathfrak{T}_{\mathfrak{g}}$-set if and only if there exist a pair $\left(\mathcal{O}_{\mathfrak{g}}, \mathcal{K}_{\mathfrak{g}}\right) \in \mathcal{T}_{\mathfrak{g}} \times \neg \mathcal{T}_{\mathfrak{g}}$ of $\mathcal{T}_{\mathfrak{g}}$-open and $\mathcal{T}_{\mathfrak{g}}$-closed sets, and a $\mathfrak{g}$-operator $\mathbf{o p}_{\mathfrak{g}}(\cdot) \in \mathcal{L}_{\mathfrak{g}}[\Omega]$ such that the following statement holds:

$$
(\exists \xi)\left[\left(\xi \in \mathcal{S}_{\mathfrak{g}}\right) \wedge\left(\left(\mathcal{S}_{\mathfrak{g}} \subseteq \mathrm{op}_{\mathfrak{g}}\left(\mathcal{O}_{\mathfrak{g}}\right)\right) \vee\left(\mathcal{S}_{\mathfrak{g}} \supseteq \neg \mathrm{op}_{\mathfrak{g}}\left(\mathcal{K}_{\mathfrak{g}}\right)\right)\right)\right]
$$

The $\mathfrak{g}$ - $\mathfrak{T}_{\mathfrak{g}}$-set $\mathcal{S}_{\mathfrak{g}} \subset \mathfrak{T}_{\mathfrak{g}}$ is said to be of category $\nu$ if and only if it belongs to the following class of $\mathfrak{g}-\nu$-T $\mathfrak{g}$-sets:

$$
\begin{aligned}
\mathfrak{g}-\nu-\mathrm{S}\left[\mathfrak{T}_{\mathfrak{g}}\right] \stackrel{\text { def }}{=} & \left\{\mathcal{S}_{\mathfrak{g}} \subset \mathfrak{T}_{\mathfrak{g}}:\left(\exists \mathcal{O}_{\mathfrak{g}}, \mathcal{K}_{\mathfrak{g}}, \mathbf{o p}_{\mathfrak{g}, \nu}(\cdot)\right)\right. \\
& {\left.\left[\left(\mathcal{S}_{\mathfrak{g}} \subseteq \mathrm{op}_{\mathfrak{g}, \nu}\left(\mathcal{O}_{\mathfrak{g}}\right)\right) \vee\left(\mathcal{S}_{\mathfrak{g}} \supseteq \neg \mathrm{op}_{\mathfrak{g}, \nu}\left(\mathcal{K}_{\mathfrak{g}}\right)\right)\right]\right\} . }
\end{aligned}
$$

It is called a $\mathfrak{g}-\nu-\mathfrak{T}_{\mathfrak{g}}$-open set if it satisfies the first property in $\mathfrak{g}-\nu$-S $\left[\mathfrak{T}_{\mathfrak{g}}\right]$ and a $\mathfrak{g}-\nu-\mathfrak{T}_{\mathfrak{g}}$-closed set if it satisfies the second property in $\mathfrak{g}-\nu-\mathrm{S}\left[\mathfrak{T}_{\mathfrak{g}}\right]$. The classes of 
$\mathfrak{g}-\nu$ - $\mathfrak{T}_{\mathfrak{g}}$-open and $\mathfrak{g}-\nu$ - $\mathfrak{T}_{\mathfrak{g}}$-closed sets, respectively, are defined by

$$
\begin{aligned}
\mathfrak{g}-\nu-\mathrm{O}\left[\mathfrak{T}_{\mathfrak{g}}\right] \stackrel{\text { def }}{=}\left\{\mathcal{S}_{\mathfrak{g}} \subset \mathfrak{T}_{\mathfrak{g}}:\left(\exists \mathcal{O}_{\mathfrak{g}}, \mathbf{o p}_{\mathfrak{g}, \nu}(\cdot)\right)\left[\mathcal{S}_{\mathfrak{g}} \subseteq \mathrm{op}_{\mathfrak{g}, \nu}\left(\mathcal{O}_{\mathfrak{g}}\right)\right]\right\}, \\
\mathfrak{g}-\nu-\mathrm{K}\left[\mathfrak{T}_{\mathfrak{g}}\right] \stackrel{\text { def }}{=}\left\{\mathcal{S}_{\mathfrak{g}} \subset \mathfrak{T}_{\mathfrak{g}}:\left(\exists \mathcal{K}_{\mathfrak{g}}, \mathbf{o p}_{\mathfrak{g}, \nu}(\cdot)\right)\left[\mathcal{S}_{\mathfrak{g}} \supseteq \neg \mathrm{op}_{\mathfrak{g}, \nu}\left(\mathcal{K}_{\mathfrak{g}}\right)\right]\right\} .
\end{aligned}
$$

From these classes, the following relation holds:

$$
\begin{aligned}
\mathfrak{g}-\mathrm{S}\left[\mathfrak{T}_{\mathfrak{g}}\right] & =\bigcup_{\nu \in I_{3}^{0}} \mathfrak{g}-\nu-\mathrm{S}\left[\mathfrak{T}_{\mathfrak{g}}\right] \\
& =\bigcup_{\nu \in I_{3}^{0}}\left(\mathfrak{g}-\nu-\mathrm{O}\left[\mathfrak{T}_{\mathfrak{g}}\right] \cup \mathfrak{g}-\nu-\mathrm{K}\left[\mathfrak{T}_{\mathfrak{g}}\right]\right) \\
& =\left(\bigcup_{\nu \in I_{3}^{0}} \mathfrak{g}-\nu-\mathrm{O}\left[\mathfrak{T}_{\mathfrak{g}}\right]\right) \cup\left(\bigcup_{\nu \in I_{3}^{0}} \mathfrak{g}-\nu-\mathrm{K}\left[\mathfrak{T}_{\mathfrak{g}}\right]\right) \\
& =\mathfrak{g}-\mathrm{O}\left[\mathfrak{T}_{\mathfrak{g}}\right] \cup \mathfrak{g}-\mathrm{K}\left[\mathfrak{T}_{\mathfrak{g}}\right] .
\end{aligned}
$$

When the subscript $\mathfrak{g}$ are omitted in almost all symbols of the above definitions, very similar definitions are derived but in a $\mathcal{T}$-space.

A $\mathfrak{T}$-set $\mathcal{S} \subset \mathfrak{T}$ in a $\mathcal{T}$-space is called a $\mathfrak{g}$ - $\mathfrak{T}$-set if and only if there exists a pair $(\mathcal{O}, \mathcal{K}) \in \mathcal{T} \times \neg \mathcal{T}$ of $\mathcal{T}$-open and $\mathcal{T}$-closed sets, and an operator op $(\cdot) \in \mathcal{L}[\Omega]$ such that the following statement holds:

$$
(\exists \xi)[(\xi \in \mathcal{S}) \wedge((\mathcal{S} \subseteq \text { op }(\mathcal{O})) \vee(\mathcal{S} \supseteq \neg \text { op }(\mathcal{K})))]
$$

The $\mathfrak{g}$-T-set $\mathcal{S} \subset \mathfrak{T}$ is said to be of category $\nu$ if and only if it belongs to the following class of $\mathfrak{g}-\nu$ - $\mathcal{T}$-sets:

$$
\begin{aligned}
\mathfrak{g}-\nu-\mathrm{S}[\mathfrak{T}] \stackrel{\text { def }}{=}\left\{\mathcal{S} \subset \mathfrak{T}:\left(\exists \mathcal{O}, \mathcal{K}, \mathbf{o p}_{\nu}(\cdot)\right)\right. \\
\left.\quad\left[\left(\mathcal{S} \subseteq \operatorname{op}_{\nu}(\mathcal{O})\right) \vee\left(\mathcal{S} \supseteq \neg \operatorname{op}_{\nu}(\mathcal{K})\right)\right]\right\} .
\end{aligned}
$$

It is called a $\mathfrak{g}-\nu$-T-open set if it satisfies the first property in $\mathfrak{g}-\nu-\mathrm{S}[\mathfrak{T}]$ and a $\mathfrak{g}-\nu$ - $\mathfrak{T}$ closed set if it satisfies the second property in $\mathfrak{g}-\nu-\mathrm{S}[\mathfrak{T}]$. The classes of $\mathfrak{g}-\nu$-T-open and $\mathfrak{g}-\nu$-T-closed sets, respectively, are defined by

$$
\begin{aligned}
& \mathfrak{g}-\nu-\mathrm{O}[\mathfrak{T}] \stackrel{\text { def }}{=}\left\{\mathcal{S} \subset \mathfrak{T}:\left(\exists \mathcal{O}, \mathbf{o p}_{\nu}(\cdot)\right)\left[\mathcal{S} \subseteq \mathrm{op}_{\nu}(\mathcal{O})\right]\right\}, \\
& \mathfrak{g}-\nu-\mathrm{K}[\mathfrak{T}] \stackrel{\text { def }}{=}\left\{\mathcal{S} \subset \mathfrak{T}:\left(\exists \mathcal{K}, \mathbf{o p}_{\nu}(\cdot)\right)\left[\mathcal{S} \supseteq \neg \mathrm{op}_{\nu}(\mathcal{K})\right]\right\} .
\end{aligned}
$$

The following relations are immediate consequences of the above definitions:

$$
\begin{aligned}
\mathfrak{g}-\mathrm{S}[\mathfrak{T}] & =\bigcup_{\nu \in I_{3}^{0}} \mathfrak{g}-\nu-\mathrm{S}[\mathfrak{T}] \\
& =\bigcup_{\nu \in I_{3}^{0}}(\mathfrak{g}-\nu-\mathrm{O}[\mathfrak{T}] \cup \mathfrak{g}-\nu-\mathrm{K}[\mathfrak{T}]) \\
& =\left(\bigcup_{\nu \in I_{3}^{0}} \mathfrak{g}-\nu-\mathrm{O}[\mathfrak{T}]\right) \cup\left(\bigcup_{\nu \in I_{3}^{0}} \mathfrak{g}-\nu-\mathrm{K}[\mathfrak{T}]\right) \\
& =\mathfrak{g}-\mathrm{O}[\mathfrak{T}] \cup \mathfrak{g}-\mathrm{K}[\mathfrak{T}] .
\end{aligned}
$$

The classes $\mathrm{O}\left[\mathfrak{T}_{\mathfrak{g}}\right]$ and $\mathrm{K}\left[\mathfrak{T}_{\mathfrak{g}}\right]$ denote the families of $\mathfrak{T}_{\mathfrak{g}}$-open and $\mathfrak{T}_{\mathfrak{g}}$-closed sets, respectively, in $\mathfrak{T}_{\mathfrak{g}}$, with $\mathrm{S}\left[\mathfrak{T}_{\mathfrak{g}}\right]=\mathrm{O}\left[\mathfrak{T}_{\mathfrak{g}}\right] \cup \mathrm{K}\left[\mathfrak{T}_{\mathfrak{g}}\right]$; the classes $\mathrm{O}[\mathfrak{T}]$ and $\mathrm{K}[\mathfrak{T}]$ denote the families of $\mathfrak{T}$-open and $\mathfrak{T}$-closed sets, respectively, in $\mathfrak{T}$, with $\mathrm{S}[\mathfrak{T}]=\mathrm{O}[\mathfrak{T}] \cup \mathrm{K}[\mathfrak{T}]$.

In regard to the above descriptions, by a $\mathfrak{g}-\mathcal{T}_{\mathfrak{g}}$-open set and a $\mathfrak{g}-\mathcal{T}_{\mathfrak{g}}$-closed set are meant a $\mathcal{T}_{\mathfrak{g}}$-open set $\mathcal{O}_{\mathfrak{g}} \in \mathcal{T}_{\mathfrak{g}}$ and a $\mathcal{T}_{\mathfrak{g}}$-closed set $\mathcal{K}_{\mathfrak{g}} \in \neg \mathcal{T}_{\mathfrak{g}}$ satisfying $\mathcal{O}_{\mathfrak{g}} \subseteq$ op $_{\mathfrak{g}}\left(\mathcal{O}_{\mathfrak{g}}\right)$ and $\mathcal{K}_{\mathfrak{g}} \supseteq \neg \mathrm{op}_{\mathfrak{g}}\left(\mathcal{K}_{\mathfrak{g}}\right)$, respectively. Likewise, by a $\mathfrak{g}-\mathcal{T}_{\mathfrak{g}}$-open set of category $\nu$ and a $\mathfrak{g}$ - $\mathcal{T}_{\mathfrak{g}}$-closed set of category $\nu$ are meant a $\mathcal{T}_{\mathfrak{g}}$-open set $\mathcal{O}_{\mathfrak{g}} \in \mathcal{T}_{\mathfrak{g}}$ and a $\mathcal{T}_{\mathfrak{g}}$-closed 
set $\mathcal{K}_{\mathfrak{g}} \in \neg \mathcal{T}_{\mathfrak{g}}$ satisfying $\mathcal{O}_{\mathfrak{g}} \subseteq \mathrm{op}_{\mathfrak{g}, \nu}\left(\mathcal{O}_{\mathfrak{g}}\right)$ and $\mathcal{K}_{\mathfrak{g}} \supseteq \neg \mathrm{op}_{\mathfrak{g}, \nu}\left(\mathcal{K}_{\mathfrak{g}}\right)$, respectively; $\mathfrak{g}-\mathcal{T}_{\mathfrak{g}}{ }^{-}$ sets of category $\nu$ will be called $\mathfrak{g}-\nu$ - $\mathcal{T}_{\mathfrak{g}}$-sets. We are now in a position to present a carefully chosen set of terms used in the theory of $\mathfrak{g}$ - $\mathfrak{T}_{\mathfrak{g}}$-separation axioms in $\mathcal{T}_{\mathfrak{g}}$-spaces.

Agreed to let $\left\langle\mathfrak{g}-\mathrm{T}_{\mathfrak{g}, \alpha}\right\rangle_{\alpha \in \Lambda}$ denote a sequence of $\mathfrak{g}-\mathrm{T}_{\mathfrak{g}, \alpha}$-axioms, indexed by the set $\Lambda \stackrel{\text { def }}{=}\{\mathrm{K}, \mathrm{F}, \mathrm{H}, \mathrm{R}, \mathrm{N}\}$, throughout the present paper, the sequence $\left\langle\mathfrak{g}-\mathfrak{T}_{\mathfrak{g}}^{(\alpha)}=\right.$ $\left.\left(\Omega, \mathfrak{g}-\mathcal{T}_{\mathfrak{g}}^{(\alpha)}\right)\right\rangle_{\alpha \in \Lambda}$ will stand for the resulting sequence of $\mathfrak{g}-\mathcal{T}_{\mathfrak{g}}^{(\alpha)}$-spaces, obtained after endowing a $\mathcal{T}_{\mathfrak{g}}$-space $\mathfrak{T}_{\mathfrak{g}}=\left(\Omega, \mathcal{T}_{\mathfrak{g}}\right)$ with $\left\langle\mathfrak{g} \text {-T } \mathrm{T}_{\mathfrak{g}, \alpha}\right\rangle_{\alpha \in \Lambda}$. Hence, the definition follows.

Definition $2.1\left(\mathfrak{g}-\mathcal{T}_{\mathfrak{g}}^{(\alpha)}\right.$-Space). A $\mathcal{T}_{\mathfrak{g}}$-space $\mathfrak{T}_{\mathfrak{g}}=\left(\Omega, \mathcal{T}_{\mathfrak{g}}\right)$ endowed with a $\mathfrak{g}$ - $T_{\mathfrak{g}, \alpha^{-}}$ axiom is called a $\mathfrak{g}-\mathcal{T}_{\mathfrak{g}}^{(\alpha)}$-space $\mathfrak{g}-\mathfrak{T}_{\mathfrak{g}}^{(\alpha)} \stackrel{\text { def }}{=}\left(\Omega, \mathfrak{g}-\mathcal{T}_{\mathfrak{g}}^{(\alpha)}\right)$.

The elements of $\left\langle\mathfrak{g}-T_{\mathfrak{g}, \alpha}\right\rangle_{\alpha \in \Lambda}$ concern the separation of points, points from $\mathfrak{g}-\mathcal{T}_{\mathfrak{g}}{ }^{-}$ open sets, and $\mathfrak{g}-\mathcal{T}_{\mathfrak{g}}$-open sets from each other. They are nicely discussed through the notions of pairwise disjoint points and $\mathfrak{g}-\mathcal{T}_{\mathfrak{g}}$-sets in a $\mathcal{T}_{\mathfrak{g}}$-space $\mathfrak{T}_{\mathfrak{g}}$. We let $\left(\mathcal{O}_{\mathfrak{g}, \xi}, \mathcal{K}_{\mathfrak{g}, \xi}\right) \in \mathcal{T}_{\mathfrak{g}} \times \neg \mathcal{T}_{\mathfrak{g}}$ denote a pair of $\mathcal{T}_{\mathfrak{g}}$-open and $\mathcal{T}_{\mathfrak{g}}$-closed sets containing the point $\xi \in \mathfrak{T}_{\mathfrak{g}}$ and let $\left(\mathcal{O}_{\mathfrak{g}, \mathcal{S}_{\mathfrak{g}}}, \mathcal{K}_{\mathfrak{g}, \mathcal{S}_{\mathfrak{g}}}\right) \in \mathcal{T}_{\mathfrak{g}} \times \neg \mathcal{T}_{\mathfrak{g}}$ denote either a pair of $\mathcal{T}_{\mathfrak{g}}$-open and $\mathcal{T}_{\mathfrak{g}}$-closed subsets or supersets of the set $\mathcal{S}_{\mathfrak{g}} \in \mathfrak{T}_{\mathfrak{g}}$, and consider the following definition.

Definition $2.2\left(\xi, \mathcal{S}_{\mathfrak{g}}\right.$-Pairwise Disjoint). Let $\mathfrak{T}_{\mathfrak{g}}=\left(\Omega, \mathcal{T}_{\mathfrak{g}}\right)$ be a $\mathcal{T}_{\mathfrak{g}}$-space. For some $\sigma \geq 0$ and $\mathcal{S}_{\mathfrak{g}} \supseteq \emptyset$, the families

$$
\begin{aligned}
& \mathfrak{g}-\mathrm{F}_{\mathrm{P}}[\sigma] \stackrel{\text { def }}{=}\left\{(\xi, \zeta) \in \mathfrak{T}_{\mathfrak{g}} \times \mathfrak{T}_{\mathfrak{g}}: \mathcal{N}_{\mathfrak{g}}(\xi, \zeta) \geq \sigma\right\}, \\
& \mathfrak{g}-\nu-\mathrm{F}_{\mathrm{O}}\left[\mathcal{S}_{\mathfrak{g}}\right] \stackrel{\text { def }}{=}\left\{\left(\mathcal{O}_{\mathfrak{g}, \xi}, \mathcal{O}_{\mathfrak{g}, \zeta}\right) \in \mathcal{T}_{\mathfrak{g}} \times \mathcal{T}_{\mathfrak{g}}: \bigcap_{\lambda=\xi, \zeta} \mathrm{op}_{\mathfrak{g}, \nu}\left(\mathcal{O}_{\mathfrak{g}, \lambda}\right) \subseteq \mathcal{S}_{\mathfrak{g}}\right\}, \\
& \mathfrak{g}-\nu-\mathrm{F}_{\mathrm{K}}\left[\mathcal{S}_{\mathfrak{g}}\right] \stackrel{\text { def }}{=}\left\{\left(\mathcal{K}_{\mathfrak{g}, \xi}, \mathcal{K}_{\mathfrak{g}, \zeta}\right) \in \neg \mathcal{T}_{\mathfrak{g}} \times \neg \mathcal{T}_{\mathfrak{g}}: \mathcal{S}_{\mathfrak{g}} \supseteq \bigcap_{\lambda=\xi, \zeta} \neg \mathrm{op}_{\mathfrak{g}, \nu}\left(\mathcal{K}_{\mathfrak{g}, \lambda}\right)\right\},
\end{aligned}
$$

respectively, denote the collections of pairwise points, and $\mathfrak{g}$ - $\mathcal{T}_{\mathfrak{g}}$-open and $\mathfrak{g}-\mathcal{T}_{\mathfrak{g}}$ closed sets of category $\nu$ in $\mathfrak{T}_{\mathfrak{g}}$. They form the collections of pairwise distinct points, and pairwise disjoint $\mathfrak{g}-\mathcal{T}_{\mathfrak{g}}$-open and $\mathfrak{g}-\mathcal{T}_{\mathfrak{g}}$-closed sets of category $\nu$ whenever $\sigma>0$ and $\mathcal{S}_{\mathfrak{g}}=\emptyset$, respectively.

Granted $\mathfrak{g}-\mathrm{F}_{\mathrm{P}}[\sigma], \mathfrak{g}-\nu-\mathrm{F}_{\mathrm{O}}\left[\mathcal{S}_{\mathfrak{g}}\right]$, and $\mathfrak{g}-\nu-\mathrm{F}_{\mathrm{K}}\left[\mathcal{S}_{\mathfrak{g}}\right]$, the elements of $\left\langle\mathfrak{g}-\nu-\mathrm{T}_{\mathfrak{g}, \alpha}\right\rangle_{\alpha \in \Lambda}$ may well be stated as thus:

Definition $2.3\left(\left\langle\mathfrak{g}-\nu \text { - } \mathrm{T}_{\mathfrak{g}, \alpha}\right\rangle_{\alpha \in \Lambda}\right.$-Axioms $)$. Let $\mathfrak{T}_{\mathfrak{g}}=\left(\Omega, \mathcal{T}_{\mathfrak{g}}\right)$ be a $\mathcal{T}_{\mathfrak{g}}$-space and suppose $\mathfrak{g}-\mathrm{F}_{\mathrm{P}}[\sigma]$, and $\mathfrak{g}-\nu-\mathrm{F}_{\mathrm{O}}\left[\mathcal{S}_{\mathfrak{g}}\right] \subseteq \mathcal{T}_{\mathfrak{g}} \times \mathcal{T}_{\mathfrak{g}}$ and $\mathfrak{g}-\nu-\mathrm{F}_{\mathrm{K}}\left[\mathcal{S}_{\mathfrak{g}}\right] \subseteq \neg \mathcal{T}_{\mathfrak{g}} \times \neg \mathcal{T}_{\mathfrak{g}}$ be given, where $\sigma \geq 0$ and $\mathcal{S}_{\mathfrak{g}} \supseteq \emptyset$. Then:

- I. $\mathfrak{g}-\nu-\mathrm{T}_{\mathfrak{g}, \mathrm{K}}$-Axiom: For every $(\xi, \zeta) \in \mathfrak{g}-\mathrm{F}_{\mathrm{P}}[\sigma>0]$, there exists a pair $\left(\mathcal{O}_{\mathfrak{g}, \xi}, \mathcal{O}_{\mathfrak{g}, \zeta}\right) \in \mathfrak{g}-\nu-\mathrm{F}_{\mathrm{O}}\left[\mathcal{S}_{\mathfrak{g}} \supset \emptyset\right]$ such that:

$$
\begin{aligned}
{\left[\left(\xi \in \mathrm{op}_{\mathfrak{g}, \nu}\left(\mathcal{O}_{\mathfrak{g}, \xi}\right)\right) \wedge\left(\zeta \notin \mathrm{op}_{\mathfrak{g}, \nu}\left(\mathcal{O}_{\mathfrak{g}, \xi}\right)\right)\right] } & \vee\left[\left(\xi \notin \mathrm{op}_{\mathfrak{g}, \nu}\left(\mathcal{O}_{\mathfrak{g}, \zeta}\right)\right)\right. \\
& \left.\wedge\left(\zeta \in \mathrm{op}_{\mathfrak{g}, \nu}\left(\mathcal{O}_{\mathfrak{g}, \zeta}\right)\right)\right] .
\end{aligned}
$$

- II. $\mathfrak{g}-\nu-\mathrm{T}_{\mathfrak{g}, \mathrm{F}^{-}}$-AXIOM: For every $(\xi, \zeta) \in \mathfrak{g}-\mathrm{F}_{\mathrm{P}}[\sigma>0]$, there exists a pair $\left(\mathcal{O}_{\mathfrak{g}, \xi}, \mathcal{O}_{\mathfrak{g}, \zeta}\right) \in \mathfrak{g}-\nu-\mathrm{F}_{\mathrm{O}}\left[\mathcal{S}_{\mathfrak{g}} \supset \emptyset\right]$ such that:

$$
\left[(\xi, \zeta) \in_{\lambda=\xi, \zeta} \mathrm{op}_{\mathfrak{g}, \nu}\left(\mathcal{O}_{\mathfrak{g}, \lambda}\right)\right] \wedge\left[(\xi, \zeta) \notin \notin_{\lambda=\zeta, \xi} \mathrm{op}_{\mathfrak{g}, \nu}\left(\mathcal{O}_{\mathfrak{g}, \lambda}\right)\right] .
$$


- III. $\mathfrak{g}-\nu$ - $\mathrm{T}_{\mathfrak{g}, \mathrm{H}}$-AXIOM: For every $(\xi, \zeta) \in \mathfrak{g}-\mathrm{F}_{\mathrm{P}}[\sigma>0]$, there exists a pair $\left(\mathcal{O}_{\mathfrak{g}, \xi}, \mathcal{O}_{\mathfrak{g}, \zeta}\right) \in \mathfrak{g}-\nu-\mathrm{F}_{\mathrm{O}}[\emptyset]$ such that:

$$
\left[\xi \in \mathrm{op}_{\mathfrak{g}, \nu}\left(\mathcal{O}_{\mathfrak{g}, \xi}\right)\right] \wedge\left[\zeta \in \mathrm{op}_{\mathfrak{g}, \nu}\left(\mathcal{O}_{\mathfrak{g}, \zeta}\right)\right] .
$$

- IV. $\mathfrak{g}-\nu-\mathrm{T}_{\mathfrak{g}, \mathrm{R}}$-AXIOM: For every $(\xi, \zeta) \in \mathfrak{g}-\mathrm{F}_{\mathrm{P}}[\sigma>0]$ and $\left(\mathcal{K}_{\mathfrak{g}, \xi}, \mathcal{K}_{\mathfrak{g}, \zeta}\right) \in$ $\mathfrak{g}-\nu-\mathrm{F}_{\mathrm{K}}[\emptyset]$ such that $(\zeta, \xi) \notin\left(\mathcal{K}_{\mathfrak{g}, \xi}, \mathcal{K}_{\mathfrak{g}, \zeta}\right)$, there exists a pair $\left(\mathcal{O}_{\mathfrak{g}, \xi}, \mathcal{O}_{\mathfrak{g}, \zeta}\right) \in$ $\mathfrak{g}-\nu-\mathrm{F}_{\mathrm{O}}[\emptyset]$ such that:

$$
\begin{aligned}
{\left[\left(\neg \mathrm{op}_{\mathfrak{g}, \nu}\left(\mathcal{K}_{\mathfrak{g}, \xi}\right)\right.\right.} & \left.\subset \mathrm{op}_{\mathfrak{g}, \nu}\left(\mathcal{O}_{\mathfrak{g}, \xi}\right)\right) \\
\left.\subset \mathrm{op}_{\mathfrak{g}, \nu}\left(\mathcal{O}_{\mathfrak{g}, \zeta}\right)\right) & \left.\wedge\left(\zeta \in \mathrm{op}_{\mathfrak{g}, \nu}\left(\mathcal{O}_{\mathfrak{g}, \zeta}\right)\right)\right] \vee\left[\left(\neg \mathrm{op}_{\mathfrak{g}, \nu}\left(\mathcal{O}_{\mathfrak{g}, \xi}\right)\right)\right]
\end{aligned}
$$

- V. $\mathfrak{g}-\nu$ - $\mathrm{T}_{\mathfrak{g}, \mathrm{N}}$-AxIOM: For every $\left(\mathcal{K}_{\mathfrak{g}, \xi}, \mathcal{K}_{\mathfrak{g}, \zeta}\right) \in \mathfrak{g}-\nu-\mathrm{F}_{\mathrm{K}}[\emptyset]$, there exists a pair $\left(\mathcal{O}_{\mathfrak{g}, \xi}, \mathcal{O}_{\mathfrak{g}, \zeta}\right) \in \mathfrak{g}-\nu-\mathrm{F}_{\mathrm{O}}[\emptyset]$ such that:

$$
\left[\mathrm{op}_{\mathfrak{g}, \nu}\left(\mathcal{O}_{\mathfrak{g}, \xi}\right) \supset \neg \mathrm{op}_{\mathfrak{g}, \nu}\left(\mathcal{K}_{\mathfrak{g}, \xi}\right)\right] \wedge\left[\mathrm{op}_{\mathfrak{g}, \nu}\left(\mathcal{O}_{\mathfrak{g}, \zeta}\right) \supset \neg \mathrm{op}_{\mathfrak{g}, \nu}\left(\mathcal{K}_{\mathfrak{g}, \zeta}\right)\right] .
$$

Granted $\left\langle\mathfrak{g}-\nu-\mathrm{T}_{\mathfrak{g}, \alpha}\right\rangle_{\alpha \in \Lambda}$, we form $\left\langle\mathfrak{g}-\mathrm{T}_{\mathfrak{g}, \alpha}\right\rangle_{\alpha \in \Lambda} \stackrel{\text { def }}{=}\left\langle\bigvee_{\nu \in I_{3}^{0}} \mathfrak{g}-\nu-\mathrm{T}_{\mathfrak{g}, \alpha}\right\rangle_{\alpha \in \Lambda}$, and define the $\mathfrak{g}-\mathrm{T}_{\mathfrak{g}, K}, \mathfrak{g}-\mathrm{T}_{\mathfrak{g}, F}, \mathfrak{g}-\mathrm{T}_{\mathfrak{g}, H}, \mathfrak{g}-\mathrm{T}_{\mathfrak{g}, \mathrm{R}}$, and $\mathfrak{g}-\mathrm{T}_{\mathfrak{g}, \mathrm{N}^{-}}$axioms as thus.

Definition $2.4\left(\left\langle\mathfrak{g}-T_{\mathfrak{g}, \alpha}\right\rangle_{\alpha \in \Lambda}\right.$-Axioms). Let $\mathfrak{T}_{\mathfrak{g}}=\left(\Omega, \mathcal{T}_{\mathfrak{g}}\right)$ be a $\mathcal{T}_{\mathfrak{g}}$-space and let $\mathfrak{g}-\mathrm{F}_{\mathrm{P}}[\sigma], \mathfrak{g}-\mathrm{F}_{\mathrm{O}}\left[\mathcal{S}_{\mathfrak{g}}\right]=\bigcup_{\nu \in I_{3}^{0}} \mathfrak{g}-\nu-\mathrm{F}_{\mathrm{O}}\left[\mathcal{S}_{\mathfrak{g}}\right] \subseteq \mathcal{T}_{\mathfrak{g}} \times \mathcal{T}_{\mathfrak{g}}, \mathfrak{g}-\mathrm{F}_{\mathrm{K}}\left[\mathcal{S}_{\mathfrak{g}}\right]=\bigcup_{\nu \in I_{3}^{0}} \mathfrak{g}-\nu-\mathrm{F}_{\mathrm{K}}\left[\mathcal{S}_{\mathfrak{g}}\right]$ $\subseteq \neg \mathcal{T}_{\mathfrak{g}} \times \neg \mathcal{T}_{\mathfrak{g}}$ be given, where $\sigma \geq 0$ and $\mathcal{S}_{\mathfrak{g}} \supseteq \emptyset$. Then:

- I. $\mathfrak{g}-\mathrm{T}_{\mathfrak{g}, \mathrm{K}}$-AxiOM: For every $(\xi, \zeta) \in \mathfrak{g}-\mathrm{F}_{\mathrm{P}}[\sigma>0]$, there exists $\left(\mathcal{O}_{\mathfrak{g}, \xi}, \mathcal{O}_{\mathfrak{g}, \zeta}\right) \in$ $\mathfrak{g}-\mathrm{F}_{\mathrm{O}}\left[\mathcal{S}_{\mathfrak{g}} \supset \emptyset\right]$ such that:

$$
\begin{aligned}
{\left[\left(\xi \in \mathrm{op}_{\mathfrak{g}}\left(\mathcal{O}_{\mathfrak{g}, \xi}\right)\right) \wedge\left(\zeta \notin \mathrm{op}_{\mathfrak{g}}\left(\mathcal{O}_{\mathfrak{g}, \xi}\right)\right)\right] } & \vee\left[\left(\xi \notin \mathrm{op}_{\mathfrak{g}}\left(\mathcal{O}_{\mathfrak{g}, \zeta}\right)\right)\right. \\
& \left.\wedge\left(\zeta \in \mathrm{op}_{\mathfrak{g}}\left(\mathcal{O}_{\mathfrak{g}, \zeta}\right)\right)\right] .
\end{aligned}
$$

- II. $\mathfrak{g}-\mathrm{T}_{\mathfrak{g}, \mathrm{F}}$-AXIOM: For every $(\xi, \zeta) \in \mathfrak{g}-\mathrm{F}_{\mathrm{P}}[\sigma>0]$, there exists a pair $\left(\mathcal{O}_{\mathfrak{g}, \xi}, \mathcal{O}_{\mathfrak{g}, \zeta}\right) \in \mathfrak{g}-\mathrm{F}_{\mathrm{O}}\left[\mathcal{S}_{\mathfrak{g}} \supset \emptyset\right]$ such that:

$$
\left[(\xi, \zeta) \in_{\lambda=\xi, \zeta} \mathrm{op}_{\mathfrak{g}}\left(\mathcal{O}_{\mathfrak{g}, \lambda}\right)\right] \wedge\left[(\xi, \zeta) \notin{ }_{\lambda=\zeta, \xi} \mathrm{op}_{\mathfrak{g}}\left(\mathcal{O}_{\mathfrak{g}, \lambda}\right)\right] .
$$

- III. $\mathfrak{g}-\mathrm{T}_{\mathfrak{g}, \mathrm{H}}$-AxiOM: For every $(\xi, \zeta) \in \mathfrak{g}-\mathrm{F}_{\mathrm{P}}[\sigma>0]$, there exists a pair $\left(\mathcal{O}_{\mathfrak{g}, \xi}, \mathcal{O}_{\mathfrak{g}, \zeta}\right) \in \mathfrak{g}-\mathrm{F}_{\mathrm{O}}[\emptyset]$ such that:

$$
\left[\xi \in \mathrm{op}_{\mathfrak{g}}\left(\mathcal{O}_{\mathfrak{g}, \xi}\right)\right] \wedge\left[\zeta \in \mathrm{op}_{\mathfrak{g}}\left(\mathcal{O}_{\mathfrak{g}, \zeta}\right)\right] .
$$

- IV. $\mathfrak{g}-\mathrm{T}_{\mathfrak{g}, \mathrm{R}}$-AxiOM: For every $(\xi, \zeta) \in \mathfrak{g}-\mathrm{F}_{\mathrm{P}}[\sigma>0]$ and $\left(\mathcal{K}_{\mathfrak{g}, \xi}, \mathcal{K}_{\mathfrak{g}, \zeta}\right) \in$ $\mathfrak{g}-\mathrm{F}_{\mathrm{K}}[\emptyset] \operatorname{such}$ that $(\zeta, \xi) \notin\left(\mathcal{K}_{\mathfrak{g}, \xi}, \mathcal{K}_{\mathfrak{g}, \zeta}\right)$, there exists $\left(\mathcal{O}_{\mathfrak{g}, \xi}, \mathcal{O}_{\mathfrak{g}, \zeta}\right) \in \mathfrak{g}-\mathrm{F}_{\mathrm{O}}[\emptyset]$ such that:

$$
\begin{aligned}
{\left[\left(\neg \mathrm{op}_{\mathfrak{g}}\left(\mathcal{K}_{\mathfrak{g}, \xi}\right) \subset \mathrm{op}_{\mathfrak{g}}\left(\mathcal{O}_{\mathfrak{g}, \xi}\right)\right)\right.} & \left.\wedge\left(\zeta \in \mathrm{op}_{\mathfrak{g}}\left(\mathcal{O}_{\mathfrak{g}, \zeta}\right)\right)\right] \vee\left[\left(\neg \mathrm{op}_{\mathfrak{g}}\left(\mathcal{K}_{\mathfrak{g}, \zeta}\right)\right.\right. \\
& \left.\left.\subset \mathrm{op}_{\mathfrak{g}}\left(\mathcal{O}_{\mathfrak{g}, \zeta}\right)\right) \wedge\left(\xi \in \mathrm{op}_{\mathfrak{g}}\left(\mathcal{O}_{\mathfrak{g}, \xi}\right)\right)\right] .
\end{aligned}
$$

- V. $\mathfrak{g}-\mathrm{T}_{\mathfrak{g}, \mathrm{N}}$-AxIOM: For every $\left(\mathcal{K}_{\mathfrak{g}, \xi}, \mathcal{K}_{\mathfrak{g}, \zeta}\right) \in \mathfrak{g}_{-} \mathrm{F}_{\mathrm{K}}[\emptyset]$, there exists a pair $\left(\mathcal{O}_{\mathfrak{g}, \xi}, \mathcal{O}_{\mathfrak{g}, \zeta}\right) \in \mathfrak{g}-\mathrm{F}_{\mathrm{O}}[\emptyset]$ such that:

$$
\left[\operatorname{op}_{\mathfrak{g}}\left(\mathcal{O}_{\mathfrak{g}, \xi}\right) \supset \neg \mathrm{op}_{\mathfrak{g}}\left(\mathcal{K}_{\mathfrak{g}, \xi}\right)\right] \wedge\left[\mathrm{op}_{\mathfrak{g}}\left(\mathcal{O}_{\mathfrak{g}, \zeta}\right) \supset \neg \mathrm{op}_{\mathfrak{g}}\left(\mathcal{K}_{\mathfrak{g}, \zeta}\right)\right] .
$$

In the following sections, the main results of the theory of $\mathfrak{g}$ - $\mathfrak{T}_{\mathfrak{g}}$-maps are presented. 


\section{Main Results}

A necessary and sufficient condition for a $\mathcal{T}_{\mathfrak{g}}$-space $\mathfrak{T}_{\mathfrak{g}}=\left(\Omega, \mathcal{T}_{\mathfrak{g}}\right)$ to be a $\mathfrak{g}$ - $\mathcal{T}_{\mathfrak{g}}^{(\mathrm{K})}$ space $\mathfrak{g}-\mathfrak{T}_{\mathfrak{g}}^{(\mathrm{K})}=\left(\Omega, \mathfrak{g}-\mathcal{T}_{\mathfrak{g}}^{(\mathrm{K})}\right)$ may be given in terms of the complementary $\mathfrak{g}$-operator $\neg \mathrm{op}_{\mathfrak{g}}: \mathcal{P}(\Omega) \longrightarrow \mathcal{P}(\Omega)$ and any pairs $(\{\xi\},\{\zeta\}) \subset \mathfrak{T}_{\mathfrak{g}} \times \mathfrak{T}_{\mathfrak{g}}$ of unit sets.

Theorem 3.1. A $\mathcal{T}_{\mathfrak{g}}$-space $\mathfrak{T}_{\mathfrak{g}}=\left(\Omega, \mathcal{T}_{\mathfrak{g}}\right)$ is said to be a $\mathfrak{g}$ - $\mathcal{T}_{\mathfrak{g}}^{(\mathrm{K})}$-space $\mathfrak{g}$ - $\mathfrak{T}_{\mathfrak{g}}^{(\mathrm{K})}=$ $\left(\Omega, \mathfrak{g}-\mathcal{T}_{\mathfrak{g}}^{(\mathrm{K})}\right)$ if and only if the following condition holds:

$$
\neg \mathrm{op}_{\mathfrak{g}}(\{\xi\}) \neq \neg \mathrm{op}_{\mathfrak{g}}(\{\zeta\}) \quad \forall(\xi, \zeta) \in \mathfrak{g}^{-\mathrm{F}_{\mathrm{P}}}[\sigma>0] .
$$

Proof. Necessity. Let the $\mathcal{T}_{\mathfrak{g}}$-space $\mathfrak{T}_{\mathfrak{g}}$ be a $\mathfrak{g}-\mathcal{T}_{\mathfrak{g}}^{(\mathrm{K})}$-space $\mathfrak{g}-\mathfrak{T}_{\mathfrak{g}}^{(\mathrm{K})}$. Then, for every $(\xi, \zeta) \in \mathfrak{g}-\mathrm{F}_{\mathrm{P}}[\sigma>0]$, there exists $\left(\mathcal{O}_{\mathfrak{g}, \xi}, \mathcal{O}_{\mathfrak{g}, \zeta}\right) \in \mathfrak{g}_{-} \mathrm{F}_{\mathrm{O}}\left[\mathcal{S}_{\mathfrak{g}} \supset \emptyset\right]$ such that:

$$
\begin{aligned}
{\left[\left(\xi \in \operatorname{op}_{\mathfrak{g}}\left(\mathcal{O}_{\mathfrak{g}, \xi}\right)\right) \wedge\left(\zeta \notin \mathrm{op}_{\mathfrak{g}}\left(\mathcal{O}_{\mathfrak{g}, \xi}\right)\right)\right] } & \vee\left[\left(\xi \notin \mathrm{op}_{\mathfrak{g}}\left(\mathcal{O}_{\mathfrak{g}, \zeta}\right)\right)\right. \\
& \left.\wedge\left(\zeta \in \operatorname{op}_{\mathfrak{g}}\left(\mathcal{O}_{\mathfrak{g}, \zeta}\right)\right)\right] .
\end{aligned}
$$

Consequently,

$$
\begin{aligned}
{\left[\left(\xi \notin \complement\left(\operatorname{op}_{\mathfrak{g}}\left(\mathcal{O}_{\mathfrak{g}, \xi}\right)\right)\right) \wedge\left(\zeta \in \complement\left(\operatorname{op}_{\mathfrak{g}}\left(\mathcal{O}_{\mathfrak{g}, \xi}\right)\right)\right)\right] } & \vee\left[\left(\xi \in \complement\left(\operatorname{op}_{\mathfrak{g}}\left(\mathcal{O}_{\mathfrak{g}, \zeta}\right)\right)\right)\right. \\
& \left.\wedge\left(\zeta \notin \complement\left(\operatorname{op}_{\mathfrak{g}}\left(\mathcal{O}_{\mathfrak{g}, \zeta}\right)\right)\right)\right]
\end{aligned}
$$

implying $\complement\left(\operatorname{op}_{\mathfrak{g}}\left(\mathcal{O}_{\mathfrak{g}, \xi}\right)\right), \complement\left(\operatorname{op}_{\mathfrak{g}}\left(\mathcal{O}_{\mathfrak{g}, \zeta}\right)\right) \in \mathfrak{g}$-K $\left[\mathfrak{T}_{\mathfrak{g}}\right]$, respectively, are $\mathfrak{g}$ - $\mathfrak{T}_{\mathfrak{g}}$-closed sets containing $\zeta \in \mathfrak{T}_{\mathfrak{g}}$ and $\xi \in \mathfrak{T}_{\mathfrak{g}}$. Thus, there exists $\left(\mathcal{K}_{\mathfrak{g}, \xi}, \mathcal{K}_{\mathfrak{g}, \zeta}\right) \in \neg \mathcal{T}_{\mathfrak{g}} \times \neg \mathcal{T}_{\mathfrak{g}}$ such that $\complement\left(\operatorname{op}_{\mathfrak{g}}\left(\mathcal{O}_{\mathfrak{g}, \xi}\right)\right) \supseteq \neg \operatorname{op}_{\mathfrak{g}}\left(\mathcal{K}_{\mathfrak{g}, \zeta}\right)$ and $\complement\left(\operatorname{op}_{\mathfrak{g}}\left(\mathcal{O}_{\mathfrak{g}, \zeta}\right)\right) \supseteq \neg \operatorname{op}_{\mathfrak{g}}\left(\mathcal{K}_{\mathfrak{g}, \xi}\right)$. But, for every $\lambda \in\{\xi, \zeta\}, \complement(\{\lambda\}) \supseteq \complement\left(\operatorname{op}_{\mathfrak{g}}(\{\lambda\})\right) \supseteq \complement\left(\operatorname{op}_{\mathfrak{g}}\left(\mathcal{O}_{\mathfrak{g}, \lambda}\right)\right)$ and $\neg \operatorname{op}_{\mathfrak{g}}\left(\mathcal{K}_{\mathfrak{g}, \lambda}\right) \supseteq \neg \mathrm{op}_{\mathfrak{g}}(\{\lambda\})$. Therefore, $\complement(\{\xi\}) \supseteq \neg \operatorname{op}_{\mathfrak{g}}(\{\zeta\})$ and $\complement(\{\zeta\}) \supseteq \neg \mathrm{op}_{\mathfrak{g}}(\{\xi\})$. Since, $\complement(\{\xi\}) \neq \complement(\{\zeta\})$, it follows that $\neg \mathrm{op}_{\mathfrak{g}}(\{\xi\}) \neq \neg \mathrm{op}_{\mathfrak{g}}(\{\zeta\})$.

Sufficiency. Conversely, suppose the condition $\neg \mathrm{op}_{\mathfrak{g}}(\{\xi\}) \neq \neg \mathrm{op}_{\mathfrak{g}}(\{\zeta\})$ holds for every $(\xi, \zeta) \in \mathfrak{g}-\mathrm{F}_{\mathrm{P}}[\sigma>0]$. Then there exists $\eta \in \mathfrak{T}_{\mathfrak{g}}$ such that

$$
\begin{aligned}
{\left[\left(\eta \in \neg \mathrm{op}_{\mathfrak{g}}(\{\xi\})\right) \wedge\left(\eta \notin \neg \mathrm{op}_{\mathfrak{g}}(\{\zeta\})\right)\right] } & \vee\left[\left(\eta \notin \neg \mathrm{op}_{\mathfrak{g}}(\{\xi\})\right)\right. \\
& \left.\wedge\left(\eta \in \neg \mathrm{op}_{\mathfrak{g}}(\{\zeta\})\right)\right] .
\end{aligned}
$$

If $\left[\xi \in \neg \mathrm{op}_{\mathfrak{g}}(\{\zeta\})\right] \wedge\left[\zeta \in \neg \mathrm{op}_{\mathfrak{g}}(\{\xi\})\right]$, then

$$
\left[\neg \mathrm{op}_{\mathfrak{g}}(\{\xi\}) \subseteq \neg \mathrm{op}_{\mathfrak{g}}(\{\zeta\})\right] \wedge\left[\neg \mathrm{op}_{\mathfrak{g}}(\{\zeta\}) \subseteq \neg \mathrm{op}_{\mathfrak{g}}(\{\xi\})\right] .
$$

Consequently,

$$
\begin{aligned}
{\left[\left(\eta \in \neg \mathrm{op}_{\mathfrak{g}}(\{\zeta\})\right) \wedge\left(\eta \notin \neg \mathrm{op}_{\mathfrak{g}}(\{\zeta\})\right)\right] } & \vee\left[\left(\eta \notin \neg \mathrm{op}_{\mathfrak{g}}(\{\xi\})\right)\right. \\
& \left.\wedge\left(\eta \in \neg \mathrm{op}_{\mathfrak{g}}(\{\xi\})\right)\right] .
\end{aligned}
$$

This is a contradiction; hence, $\left[\xi \notin \neg \mathrm{op}_{\mathfrak{g}}(\{\zeta\})\right] \wedge\left[\zeta \notin \neg \mathrm{op}_{\mathfrak{g}}(\{\xi\})\right]$, implying

$$
\left[\xi \in \complement\left(\neg \mathrm{op}_{\mathfrak{g}}(\{\zeta\})\right)\right] \wedge\left[\zeta \in \complement\left(\neg \mathrm{op}_{\mathfrak{g}}(\{\xi\})\right)\right] .
$$

Since $\complement\left(\neg \operatorname{op}_{\mathfrak{g}}(\{\zeta\})\right), \complement\left(\neg \mathrm{op}_{\mathfrak{g}}(\{\xi\})\right) \in \mathfrak{g}$-O $\left[\mathfrak{T}_{\mathfrak{g}}\right]$, respectively, are $\mathfrak{g}$-Tg $\mathfrak{T}_{\mathfrak{g}}$-open sets containing $\xi \in \mathfrak{T}_{\mathfrak{g}}$ and $\zeta \in \mathfrak{T}_{\mathfrak{g}}$, there exists $\left(\mathcal{O}_{\mathfrak{g}, \xi}, \mathcal{O}_{\mathfrak{g}, \zeta}\right) \in \mathcal{T}_{\mathfrak{g}} \times \mathcal{T}_{\mathfrak{g}}$ such that $\complement\left(\neg \mathrm{op}_{\mathfrak{g}}(\{\zeta\})\right) \subseteq \operatorname{op}_{\mathfrak{g}}\left(\mathcal{O}_{\mathfrak{g}, \xi}\right)$ and $\complement\left(\neg \mathrm{op}_{\mathfrak{g}}(\{\xi\})\right) \subseteq \mathrm{op}_{\mathfrak{g}}\left(\mathcal{O}_{\mathfrak{g}, \zeta}\right)$. Hence, for every $(\xi, \zeta) \in \mathfrak{g}-\mathrm{F}_{\mathrm{P}}[\sigma>0]$, there exists $\left(\mathcal{O}_{\mathfrak{g}, \xi}, \mathcal{O}_{\mathfrak{g}, \zeta}\right) \in \mathfrak{g}-\mathrm{F}_{\mathrm{O}}\left[\mathcal{S}_{\mathfrak{g}} \supset \emptyset\right]$ such that:

$$
\begin{aligned}
{\left[\left(\xi \in \mathrm{op}_{\mathfrak{g}}\left(\mathcal{O}_{\mathfrak{g}, \xi}\right)\right) \wedge\left(\zeta \notin \mathrm{op}_{\mathfrak{g}}\left(\mathcal{O}_{\mathfrak{g}, \xi}\right)\right)\right] } & \vee\left[\left(\xi \notin \mathrm{op}_{\mathfrak{g}}\left(\mathcal{O}_{\mathfrak{g}, \zeta}\right)\right)\right. \\
& \left.\wedge\left(\zeta \in \mathrm{op}_{\mathfrak{g}}\left(\mathcal{O}_{\mathfrak{g}, \zeta}\right)\right)\right] .
\end{aligned}
$$


Therefore, $\mathfrak{T}_{\mathfrak{g}}=\left(\Omega, \mathcal{T}_{\mathfrak{g}}\right)$ is a $\mathfrak{g}-\mathcal{T}_{\mathfrak{g}}^{(\mathrm{K})}$-space $\mathfrak{g}-\mathfrak{T}_{\mathfrak{g}}^{(\mathrm{K})}=\left(\Omega, \mathfrak{g}-\mathcal{T}_{\mathfrak{g}}^{(\mathrm{K})}\right)$; this completes the proof of the theorem.

A necessary and sufficient condition for a $\mathcal{T}_{\mathfrak{g}}$-space $\mathfrak{T}_{\mathfrak{g}}=\left(\Omega, \mathcal{T}_{\mathfrak{g}}\right)$ to be a $\mathfrak{g}-\mathcal{T}_{\mathfrak{g}}^{(\mathrm{F})}$ space $\mathfrak{g}-\mathfrak{T}_{\mathfrak{g}}^{(\mathrm{F})}=\left(\Omega, \mathfrak{g}-\mathcal{T}_{\mathfrak{g}}^{(\mathrm{F})}\right)$ may be given in terms of the complementary $\mathfrak{g}$-operator $\neg \mathrm{op}_{\mathfrak{g}}: \mathcal{P}(\Omega) \longrightarrow \mathcal{P}(\Omega)$ and a unit set $\{\xi\} \subset \mathfrak{T}_{\mathfrak{g}}$.

Theorem 3.2. A $\mathcal{T}_{\mathfrak{g}}$-space $\mathfrak{T}_{\mathfrak{g}}=\left(\Omega, \mathcal{T}_{\mathfrak{g}}\right)$ is said to be a $\mathfrak{g}-\mathcal{T}_{\mathfrak{g}}^{(\mathrm{F})}$-space $\mathfrak{g}-\mathfrak{T}_{\mathfrak{g}}^{(\mathrm{F})}=$ $\left(\Omega, \mathfrak{g}-\mathcal{T}_{\mathfrak{g}}^{(\mathrm{F})}\right)$ if and only if the following condition holds:

$$
\{\xi\} \supseteq \neg \operatorname{op}_{\mathfrak{g}}(\{\xi\}) \quad \forall \xi \in \mathfrak{T}_{\mathfrak{g}} .
$$

Proof. Necessity. Let the $\mathcal{T}_{\mathfrak{g}}$-space $\mathfrak{T}_{\mathfrak{g}}$ be a $\mathfrak{g}-\mathcal{T}_{\mathfrak{g}}^{(\mathrm{F})}$-space $\mathfrak{g}$ - $\mathfrak{T}_{\mathfrak{g}}^{(\mathrm{F})}$. Then, for every $(\xi, \zeta) \in \mathfrak{g}-\mathrm{F}_{\mathrm{P}}[\sigma>0]$, there exists $\left(\mathcal{O}_{\mathfrak{g}, \xi}, \mathcal{O}_{\mathfrak{g}, \zeta}\right) \in \mathfrak{g}_{-} \mathrm{F}_{\mathrm{O}}\left[\mathcal{S}_{\mathfrak{g}} \supset \emptyset\right]$ such that:

$$
\left[(\xi, \zeta) \in{ }_{\lambda=\xi, \zeta} \mathrm{op}_{\mathfrak{g}}\left(\mathcal{O}_{\mathfrak{g}, \lambda}\right)\right] \wedge\left[(\xi, \zeta) \notin \lambda=\zeta, \xi \mathrm{op}_{\mathfrak{g}}\left(\mathcal{O}_{\mathfrak{g}, \lambda}\right)\right] .
$$

Consequently,

$$
\left[(\xi, \zeta) \notin{ }_{\lambda=\xi, \zeta} \complement\left(\operatorname{op}_{\mathfrak{g}}\left(\mathcal{O}_{\mathfrak{g}, \lambda}\right)\right)\right] \wedge\left[(\xi, \zeta) \in_{\lambda=\zeta, \xi} \complement\left(\mathrm{op}_{\mathfrak{g}}\left(\mathcal{O}_{\mathfrak{g}, \lambda}\right)\right)\right] .
$$

Since, for every $\lambda \in\{\xi, \zeta\}, \complement\left(\operatorname{op}_{\mathfrak{g}}\left(\mathcal{O}_{\mathfrak{g}, \lambda}\right)\right) \in \mathfrak{g}-\mathrm{K}\left[\mathfrak{T}_{\mathfrak{g}}\right]$ is a $\mathfrak{g}$ - $\mathfrak{T}_{\mathfrak{g}}$-closed set, there exists $\left(\mathcal{K}_{\mathfrak{g}, \xi}, \mathcal{K}_{\mathfrak{g}, \zeta}\right) \in \neg \mathcal{T}_{\mathfrak{g}} \times \neg \mathcal{T}_{\mathfrak{g}}$ such that $\complement\left(\operatorname{op}_{\mathfrak{g}}\left(\mathcal{O}_{\mathfrak{g}, \xi}\right)\right) \supseteq \neg \operatorname{op}_{\mathfrak{g}}\left(\mathcal{K}_{\mathfrak{g}, \zeta}\right)$ and $\complement\left(\operatorname{op}_{\mathfrak{g}}\left(\mathcal{O}_{\mathfrak{g}, \zeta}\right)\right) \supseteq$ $\neg \operatorname{op}_{\mathfrak{g}}\left(\mathcal{K}_{\mathfrak{g}, \xi}\right)$. But, for every $\lambda \in\{\xi, \zeta\}, \neg \operatorname{op}_{\mathfrak{g}}\left(\mathcal{K}_{\mathfrak{g}, \lambda}\right) \supseteq \neg \operatorname{op}_{\mathfrak{g}}(\{\lambda\})$. Therefore

$$
\left[(\xi, \zeta) \in_{\lambda=\xi, \zeta} \neg \mathrm{op}_{\mathfrak{g}}(\{\lambda\})\right] \wedge\left[(\xi, \zeta) \notin \lambda=\zeta, \xi \neg \mathrm{op}_{\mathfrak{g}}(\{\lambda\})\right] .
$$

Hence, for every $\xi \in \mathfrak{T}_{\mathfrak{g}},\{\xi\} \supseteq \neg \mathrm{op}_{\mathfrak{g}}(\{\xi\})$.

Sufficiency. Conversely, suppose the condition $\{\xi\} \supseteq \neg \mathrm{op}_{\mathfrak{g}}(\{\xi\})$ holds for every $\xi \in \mathfrak{T}_{\mathfrak{g}}$. Let $(\xi, \zeta) \in \mathfrak{T}_{\mathfrak{g}} \times \mathfrak{T}_{\mathfrak{g}}$ such that $\xi \neq \zeta$. Then

$$
\left[(\xi, \zeta) \notin{ }_{\lambda=\xi, \zeta} \complement(\{\lambda\})\right] \wedge\left[(\xi, \zeta) \in_{\lambda=\zeta, \xi} \complement(\{\lambda\})\right] .
$$

But, for every $\lambda \in\{\xi, \zeta\}, \complement(\{\lambda\}) \subseteq \complement\left(\neg \mathrm{op}_{\mathfrak{g}}(\{\lambda\})\right)$, and $\complement\left(\neg \mathrm{op}_{\mathfrak{g}}(\{\lambda\})\right) \in \mathfrak{g}-\mathrm{O}\left[\mathfrak{T}_{\mathfrak{g}}\right]$ is a $\mathfrak{g}$ - $\mathfrak{T}_{\mathfrak{g}}$-open set. Thus, there exists $\left(\mathcal{O}_{\mathfrak{g}, \xi}, \mathcal{O}_{\mathfrak{g}, \zeta}\right) \in \mathcal{T}_{\mathfrak{g}} \times \mathcal{T}_{\mathfrak{g}}$ such that $\complement\left(\neg \operatorname{op}_{\mathfrak{g}}(\{\xi\})\right) \subseteq$ $\operatorname{op}_{\mathfrak{g}}\left(\mathcal{O}_{\mathfrak{g}, \zeta}\right)$ and $\complement\left(\neg \mathrm{op}_{\mathfrak{g}}(\{\zeta\})\right) \subseteq \mathrm{op}_{\mathfrak{g}}\left(\mathcal{O}_{\mathfrak{g}, \xi}\right)$. By substitution, it thus follows that, for every $(\xi, \zeta) \in \mathfrak{g}-\mathrm{F}_{\mathrm{P}}[\sigma>0]$, there exists $\left(\mathcal{O}_{\mathfrak{g}, \xi}, \mathcal{O}_{\mathfrak{g}, \zeta}\right) \in \mathfrak{g}-\mathrm{F}_{\mathrm{O}}\left[\mathcal{S}_{\mathfrak{g}} \supset \emptyset\right]$ such that:

$$
\left[(\xi, \zeta) \in_{\lambda=\xi, \zeta} \mathrm{op}_{\mathfrak{g}}\left(\mathcal{O}_{\mathfrak{g}, \lambda}\right)\right] \wedge\left[(\xi, \zeta) \notin_{\lambda=\zeta, \xi} \mathrm{Op}_{\mathfrak{g}}\left(\mathcal{O}_{\mathfrak{g}, \lambda}\right)\right]
$$

Therefore, $\mathfrak{T}_{\mathfrak{g}}=\left(\Omega, \mathcal{T}_{\mathfrak{g}}\right)$ is a $\mathfrak{g}-\mathcal{T}_{\mathfrak{g}}^{(\mathrm{F})}$-space $\mathfrak{g}-\mathfrak{T}_{\mathfrak{g}}^{(\mathrm{F})}=\left(\Omega, \mathfrak{g}-\mathcal{T}_{\mathfrak{g}}^{(\mathrm{F})}\right)$; this completes the proof of the theorem.

Proposition 1. If $\mathfrak{T}_{\mathfrak{g}}=\left(\Omega, \mathcal{T}_{\mathfrak{g}}\right)$ is a $\mathfrak{g}-\mathcal{T}_{\mathfrak{g}}^{(\mathrm{F})}$-space $\mathfrak{g}-\mathfrak{T}_{\mathfrak{g}}^{(\mathrm{F})}=\left(\Omega, \mathfrak{g}-\mathcal{T}_{\mathfrak{g}}^{(\mathrm{F})}\right)$, then it is a $\mathfrak{g}-\mathcal{T}_{\mathfrak{g}}^{(\mathrm{K})}$-space $\mathfrak{g}-\mathfrak{T}_{\mathfrak{g}}^{(\mathrm{K})}=\left(\Omega, \mathfrak{g}-\mathcal{T}_{\mathfrak{g}}^{(\mathrm{K})}\right)$.

Proof. Let $\mathfrak{T}_{\mathfrak{g}}=\left(\Omega, \mathcal{T}_{\mathfrak{g}}\right)$ be a $\mathfrak{g}-\mathcal{T}_{\mathfrak{g}}^{(\mathrm{F})}$-space $\mathfrak{g}-\mathfrak{T}_{\mathfrak{g}}^{(\mathrm{F})}=\left(\Omega, \mathfrak{g}-\mathcal{T}_{\mathfrak{g}}^{(\mathrm{F})}\right)$. Then, for every $(\xi, \zeta) \in \mathfrak{g}-\mathrm{F}_{\mathrm{P}}[\sigma>0]$, there exists a pair $\left(\mathcal{O}_{\mathfrak{g}, \xi}, \mathcal{O}_{\mathfrak{g}, \zeta}\right) \in \mathfrak{g}-\mathrm{F}_{\mathrm{O}}\left[\mathcal{S}_{\mathfrak{g}} \supset \emptyset\right]$ such that:

$$
\left[(\xi, \zeta) \in{ }_{\lambda=\xi, \zeta} \mathrm{op}_{\mathfrak{g}}\left(\mathcal{O}_{\mathfrak{g}, \lambda}\right)\right] \wedge\left[(\xi, \zeta) \notin \lambda=\zeta, \xi \mathrm{op}_{\mathfrak{g}}\left(\mathcal{O}_{\mathfrak{g}, \lambda}\right)\right]
$$

Set $\mathrm{P}(\xi, \zeta)=\left(\xi \in \mathrm{op}_{\mathfrak{g}}\left(\mathcal{O}_{\mathfrak{g}, \xi}\right)\right) \wedge\left(\zeta \notin \mathrm{op}_{\mathfrak{g}}\left(\mathcal{O}_{\mathfrak{g}, \xi}\right)\right)$. Then, the above logical statement is equivalent to $\mathrm{P}(\xi, \zeta) \wedge \mathrm{P}(\zeta, \xi)$. But, logically,

$$
\mathrm{P}(\xi, \zeta) \vee \mathrm{P}(\zeta, \xi)=\mathrm{P}(\xi, \zeta) \vee \mathrm{P}(\zeta, \xi) \vee(\mathrm{P}(\xi, \zeta) \wedge \mathrm{P}(\zeta, \xi))
$$


Consequently, $\mathrm{P}(\xi, \zeta) \vee \mathrm{P}(\zeta, \xi) \longleftarrow \mathrm{P}(\xi, \zeta) \wedge \mathrm{P}(\zeta, \xi)$, from which it then follows that, if $\mathfrak{T}_{\mathfrak{g}}=\left(\Omega, \mathcal{T}_{\mathfrak{g}}\right)$ is a $\mathfrak{g}-\mathcal{T}_{\mathfrak{g}}^{(\mathrm{F})}$-space $\mathfrak{g}-\mathfrak{T}_{\mathfrak{g}}^{(\mathrm{F})}=\left(\Omega, \mathfrak{g}-\mathcal{T}_{\mathfrak{g}}^{(\mathrm{F})}\right)$, then for every $(\xi, \zeta) \in$ $\mathfrak{g}-\mathrm{F}_{\mathrm{P}}[\sigma>0]$, there exists $\left(\mathcal{O}_{\mathfrak{g}, \xi}, \mathcal{O}_{\mathfrak{g}, \zeta}\right) \in \mathfrak{g}-\mathrm{F}_{\mathrm{O}}\left[\mathcal{S}_{\mathfrak{g}} \supset \emptyset\right]$ such that:

$$
\begin{aligned}
{\left[\left(\xi \in \mathrm{op}_{\mathfrak{g}}\left(\mathcal{O}_{\mathfrak{g}, \xi}\right)\right) \wedge\left(\zeta \notin \mathrm{op}_{\mathfrak{g}}\left(\mathcal{O}_{\mathfrak{g}, \xi}\right)\right)\right] } & \vee\left[\left(\xi \notin \mathrm{op}_{\mathfrak{g}}\left(\mathcal{O}_{\mathfrak{g}, \zeta}\right)\right)\right. \\
& \left.\wedge\left(\zeta \in \mathrm{op}_{\mathfrak{g}}\left(\mathcal{O}_{\mathfrak{g}, \zeta}\right)\right)\right],
\end{aligned}
$$

the logical statement characterising $\mathfrak{T}_{\mathfrak{g}}=\left(\Omega, \mathcal{T}_{\mathfrak{g}}\right)$ as a $\mathfrak{g}-\mathcal{T}_{\mathfrak{g}}^{(\mathrm{K})}$-space $\mathfrak{g}$ - $\mathfrak{T}_{\mathfrak{g}}^{(\mathrm{K})}=$ $\left(\Omega, \mathfrak{g}-\mathcal{T}_{\mathfrak{g}}^{(\mathrm{K})}\right)$.

A necessary and sufficient condition for a $\mathcal{T}_{\mathfrak{g}}$-space $\mathfrak{T}_{\mathfrak{g}}=\left(\Omega, \mathcal{T}_{\mathfrak{g}}\right)$ to be a $\mathfrak{g}-\mathcal{T}_{\mathfrak{g}}^{(\mathrm{H})}$ space $\mathfrak{g}-\mathfrak{T}_{\mathfrak{g}}^{(\mathrm{H})}=\left(\Omega, \mathfrak{g}-\mathcal{T}_{\mathfrak{g}}^{(\mathrm{H})}\right)$ may be given in terms of the $\mathfrak{g}$-operator op $\mathfrak{g}: \mathcal{P}(\Omega) \longrightarrow$ $\mathcal{P}(\Omega)$, a unit set $\{\xi\} \subset \mathfrak{T}_{\mathfrak{g}}$, and $\mathcal{T}_{\mathfrak{g}}$-closed sets.

Theorem 3.3. A $\mathcal{T}_{\mathfrak{g}}$-space $\mathfrak{T}_{\mathfrak{g}}=\left(\Omega, \mathcal{T}_{\mathfrak{g}}\right)$ is said to be a $\mathfrak{g}-\mathcal{T}_{\mathfrak{g}}^{(\mathrm{H})}$-space $\mathfrak{g}-\mathfrak{T}_{\mathfrak{g}}^{(\mathrm{H})}=$ $\left(\Omega, \mathfrak{g}-\mathcal{T}_{\mathfrak{g}}^{(\mathrm{H})}\right)$ if and only if the following conditions hold:

$$
\{\xi\}=\bigcap_{\mathcal{K}_{\mathfrak{g}, \zeta} \in \neg \mathcal{T}_{\mathfrak{g}}} \neg \mathrm{op}_{\mathfrak{g}}\left(\mathcal{K}_{\mathfrak{g}, \zeta}\right) \quad \forall \xi \in \mathfrak{T}_{\mathfrak{g}} .
$$

Proof. Necessity. Let the $\mathcal{T}_{\mathfrak{g}}$-space $\mathfrak{T}_{\mathfrak{g}}$ be a $\mathfrak{g}-\mathcal{T}_{\mathfrak{g}}^{(\mathrm{H})}$-space $\mathfrak{g}$ - $\mathfrak{T}_{\mathfrak{g}}^{(\mathrm{H})}$. Then, for every $(\xi, \zeta) \in \mathfrak{g}-\mathrm{F}_{\mathrm{P}}[\sigma>0]$, there exists $\left(\mathcal{O}_{\mathfrak{g}, \xi}, \mathcal{O}_{\mathfrak{g}, \zeta}\right) \in \mathfrak{g}-\mathrm{F}_{\mathrm{O}}[\emptyset]$ such that:

$$
\left[\xi \in \mathrm{op}_{\mathfrak{g}}\left(\mathcal{O}_{\mathfrak{g}, \xi}\right)\right] \wedge\left[\zeta \in \mathrm{op}_{\mathfrak{g}}\left(\mathcal{O}_{\mathfrak{g}, \zeta}\right)\right]
$$

Consequently,

$$
\left[\xi \notin \complement\left(\mathrm{op}_{\mathfrak{g}}\left(\mathcal{O}_{\mathfrak{g}, \xi}\right)\right)\right] \wedge\left[\zeta \notin \complement\left(\mathrm{op}_{\mathfrak{g}}\left(\mathcal{O}_{\mathfrak{g}, \zeta}\right)\right)\right] .
$$

But, for every $\lambda \in\{\xi, \zeta\}, \complement\left(\operatorname{op}_{\mathfrak{g}}\left(\mathcal{O}_{\mathfrak{g}, \lambda}\right)\right) \in \mathfrak{g}-\mathrm{K}\left[\mathfrak{T}_{\mathfrak{g}}\right]$ is a $\mathfrak{g}-\mathfrak{T}_{\mathfrak{g}}$-closed set. Consequently, there exists a pair $\left(\mathcal{K}_{\mathfrak{g}, \xi}, \mathcal{K}_{\mathfrak{g}, \zeta}\right) \in \neg \mathcal{T}_{\mathfrak{g}} \times \neg \mathcal{T}_{\mathfrak{g}}$ such that the relations $\complement\left(\operatorname{op}_{\mathfrak{g}}\left(\mathcal{O}_{\mathfrak{g}, \xi}\right)\right) \supseteq \neg \mathrm{op}_{\mathfrak{g}}\left(\mathcal{K}_{\mathfrak{g}, \zeta}\right)$ and $\complement\left(\operatorname{op}_{\mathfrak{g}}\left(\mathcal{O}_{\mathfrak{g}, \zeta}\right)\right) \supseteq \neg \mathrm{op}_{\mathfrak{g}}\left(\mathcal{K}_{\mathfrak{g}, \xi}\right)$ hold true. Therefore, the relations $\xi \in \complement(\{\zeta\}) \supseteq \neg \mathrm{op}_{\mathfrak{g}}(\{\xi\})$ and $\zeta \in \complement(\{\xi\}) \supseteq \neg \mathrm{op}_{\mathfrak{g}}(\{\zeta\})$ are true for all $(\xi, \zeta) \in \mathfrak{T}_{\mathfrak{g}} \times \mathfrak{T}_{\mathfrak{g}}$. But, $\complement(\{\xi\})=\bigcup_{\mathcal{O}_{\mathfrak{g}, \zeta} \in \mathcal{T}_{\mathfrak{g}}}$ op $\mathfrak{g}\left(\mathcal{O}_{\mathfrak{g}, \zeta}\right)$ and hence,

$$
\{\xi\}=\complement(\complement(\{\xi\}))=\bigcap_{\mathcal{O}_{\mathfrak{g}, \zeta} \in \mathcal{T}_{\mathfrak{g}}} \complement\left(\mathrm{op}_{\mathfrak{g}}\left(\mathcal{O}_{\mathfrak{g}, \zeta}\right)\right)=\bigcap_{\mathcal{K}_{\mathfrak{g}, \zeta} \in \neg \mathcal{T}_{\mathfrak{g}}} \neg \mathrm{op}_{\mathfrak{g}}\left(\mathcal{K}_{\mathfrak{g}, \zeta}\right) \quad \forall \xi \in \mathfrak{T}_{\mathfrak{g}} .
$$

Sufficiency. Conversely, suppose $\{\xi\}=\bigcap_{\mathcal{K}_{\mathfrak{g}, \zeta} \in \neg \mathcal{T}_{\mathfrak{g}}} \neg \mathrm{op}_{\mathfrak{g}}\left(\mathcal{K}_{\mathfrak{g}, \zeta}\right)$ holds for all $\xi \in \mathfrak{T}_{\mathfrak{g}}$. Then, there exists a $\mathcal{K}_{\mathfrak{g}, \xi} \in \neg \mathcal{T}_{\mathfrak{g}}$ such that $\zeta \notin \neg \mathrm{op}_{\mathfrak{g}}\left(\mathcal{K}_{\mathfrak{g}, \xi}\right)$. Since $\neg \mathrm{op}_{\mathfrak{g}}\left(\mathcal{K}_{\mathfrak{g}, \xi}\right) \in$ $\mathfrak{g}-\mathrm{K}\left[\mathfrak{T}_{\mathfrak{g}}\right]$ is a $\mathfrak{g}-\mathfrak{T}_{\mathfrak{g}}$-closed set, there exists a $\mathfrak{g}$ - $\mathfrak{T}_{\mathfrak{g}}$-open set op $\mathfrak{g}_{\mathfrak{g}}\left(\mathcal{O}_{\mathfrak{g}, \xi}\right) \in \mathfrak{g}$-O $\left[\mathfrak{T}_{\mathfrak{g}}\right]$ such that $\xi \in \operatorname{op}_{\mathfrak{g}}\left(\mathcal{O}_{\mathfrak{g}, \xi}\right) \subseteq \neg \mathrm{op}_{\mathfrak{g}}\left(\mathcal{K}_{\mathfrak{g}, \xi}\right)$. But, since $\complement\left(\neg \mathrm{op}_{\mathfrak{g}}\left(\mathcal{K}_{\mathfrak{g}, \xi}\right)\right) \in \mathfrak{g}-\mathrm{O}\left[\mathfrak{T}_{\mathfrak{g}}\right]$ is a $\mathfrak{g}-\mathfrak{T}_{\mathfrak{g}}$ open set containing $\zeta \in \mathfrak{T}_{\mathfrak{g}}$, it follows that $\operatorname{op}_{\mathfrak{g}}\left(\mathcal{O}_{\mathfrak{g}, \xi}\right), \complement\left(\neg \mathrm{op}_{\mathfrak{g}}\left(\mathcal{K}_{\mathfrak{g}, \xi}\right)\right) \in \mathfrak{g}-\mathrm{O}\left[\mathfrak{T}_{\mathfrak{g}}\right]$ are disjoint $\mathfrak{g}$ - $\mathfrak{T}_{\mathfrak{g}}$-open sets. Thus, for every $(\xi, \zeta) \in \mathfrak{g}$ - $\mathrm{F}_{\mathrm{P}}[\sigma>0]$, there exists $\left(\mathcal{O}_{\mathfrak{g}, \xi}, \mathcal{O}_{\mathfrak{g}, \zeta}\right) \in \mathfrak{g}-\mathrm{F}_{\mathrm{O}}[\emptyset]$ such that:

$$
\left[\xi \in \mathrm{op}_{\mathfrak{g}}\left(\mathcal{O}_{\mathfrak{g}, \xi}\right)\right] \wedge\left[\zeta \in \mathrm{op}_{\mathfrak{g}}\left(\mathcal{O}_{\mathfrak{g}, \zeta}\right)\right]
$$

Therefore, $\mathfrak{T}_{\mathfrak{g}}=\left(\Omega, \mathcal{T}_{\mathfrak{g}}\right)$ is a $\mathfrak{g}-\mathcal{T}_{\mathfrak{g}}^{(\mathrm{H})}$-space $\mathfrak{g}-\mathfrak{T}_{\mathfrak{g}}^{(\mathrm{H})}=\left(\Omega, \mathfrak{g}-\mathcal{T}_{\mathfrak{g}}^{(\mathrm{H})}\right)$; this completes the proof of the theorem.

Proposition 2. If $\mathfrak{T}_{\mathfrak{g}}=\left(\Omega, \mathcal{T}_{\mathfrak{g}}\right)$ is a $\mathfrak{g}-\mathcal{T}_{\mathfrak{g}}^{(\mathrm{H})}$-space $\mathfrak{g}-\mathfrak{T}_{\mathfrak{g}}^{(\mathrm{H})}=\left(\Omega, \mathfrak{g}-\mathcal{T}_{\mathfrak{g}}^{(\mathrm{H})}\right)$, then it is a $\mathfrak{g}-\mathcal{T}_{\mathfrak{g}}^{(\mathrm{F})}$-space $\mathfrak{g}-\mathfrak{T}_{\mathfrak{g}}^{(\mathrm{F})}=\left(\Omega, \mathfrak{g}-\mathcal{T}_{\mathfrak{g}}^{(\mathrm{F})}\right)$. 
Proof. Let $\mathfrak{T}_{\mathfrak{g}}=\left(\Omega, \mathcal{T}_{\mathfrak{g}}\right)$ be a $\mathfrak{g}-\mathcal{T}_{\mathfrak{g}}^{(\mathrm{H})}$-space $\mathfrak{g}-\mathfrak{T}_{\mathfrak{g}}^{(\mathrm{H})}=\left(\Omega, \mathfrak{g}-\mathcal{T}_{\mathfrak{g}}^{(\mathrm{H})}\right)$. Then, for every $(\xi, \zeta) \in \mathfrak{g}-\mathrm{F}_{\mathrm{P}}[\sigma>0]$, there exists a pair $\left(\mathcal{O}_{\mathfrak{g}, \xi}, \mathcal{O}_{\mathfrak{g}, \zeta}\right) \in \mathfrak{g}-\mathrm{F}_{\mathrm{O}}[\emptyset]$ such that $[\xi \in$ $\left.\operatorname{op}_{\mathfrak{g}}\left(\mathcal{O}_{\mathfrak{g}, \xi}\right)\right] \wedge\left[\zeta \in \operatorname{op}_{\mathfrak{g}}\left(\mathcal{O}_{\mathfrak{g}, \zeta}\right)\right]$. But since $\left(\mathcal{O}_{\mathfrak{g}, \xi}, \mathcal{O}_{\mathfrak{g}, \zeta}\right) \in \mathfrak{g}-\mathrm{F}_{\mathrm{O}}[\emptyset] \subset \mathfrak{g}-\mathrm{F}_{\mathrm{O}}\left[\mathcal{S}_{\mathfrak{g}} \supset \emptyset\right]$ and

$$
\begin{aligned}
{\left[\xi \in \mathrm{op}_{\mathfrak{g}}\left(\mathcal{O}_{\mathfrak{g}, \xi}\right)\right] } & \wedge\left[\zeta \in \mathrm{op}_{\mathfrak{g}}\left(\mathcal{O}_{\mathfrak{g}, \zeta}\right)\right] \\
\Leftrightarrow \quad\left[\left(\xi \in \mathrm{op}_{\mathfrak{g}}\left(\mathcal{O}_{\mathfrak{g}, \xi}\right)\right) \wedge\left(\zeta \in \mathrm{op}_{\mathfrak{g}}\left(\mathcal{O}_{\mathfrak{g}, \zeta}\right)\right)\right] & \wedge\left[\left(\xi \notin \mathrm{op}_{\mathfrak{g}}\left(\mathcal{O}_{\mathfrak{g}, \zeta}\right)\right)\right. \\
& \left.\wedge\left(\zeta \notin \mathrm{op}_{\mathfrak{g}}\left(\mathcal{O}_{\mathfrak{g}, \xi}\right)\right)\right],
\end{aligned}
$$

it follows that, if $\mathfrak{T}_{\mathfrak{g}}=\left(\Omega, \mathcal{T}_{\mathfrak{g}}\right)$ is a $\mathfrak{g}-\mathcal{T}_{\mathfrak{g}}^{(\mathrm{H})}$-space $\mathfrak{g}-\mathfrak{T}_{\mathfrak{g}}^{(\mathrm{H})}=\left(\Omega, \mathfrak{g}-\mathcal{T}_{\mathfrak{g}}^{(\mathrm{H})}\right)$, then for every $(\xi, \zeta) \in \mathfrak{g}-\mathrm{F}_{\mathrm{P}}[\sigma>0]$, there exists a pair $\left(\mathcal{O}_{\mathfrak{g}, \xi}, \mathcal{O}_{\mathfrak{g}, \zeta}\right) \in \mathfrak{g}-\mathrm{F}_{\mathrm{O}}\left[\mathcal{S}_{\mathfrak{g}} \supset \emptyset\right]$ such that:

$$
\left[(\xi, \zeta) \in_{\lambda=\xi, \zeta} \mathrm{op}_{\mathfrak{g}}\left(\mathcal{O}_{\mathfrak{g}, \lambda}\right)\right] \wedge\left[(\xi, \zeta) \notin{ }_{\lambda=\zeta, \xi} \mathrm{op}_{\mathfrak{g}}\left(\mathcal{O}_{\mathfrak{g}, \lambda}\right)\right] .
$$

Hence, if $\mathfrak{T}_{\mathfrak{g}}=\left(\Omega, \mathcal{T}_{\mathfrak{g}}\right)$ is a $\mathfrak{g}-\mathcal{T}_{\mathfrak{g}}^{(\mathrm{H})}$-space $\mathfrak{g}-\mathfrak{T}_{\mathfrak{g}}^{(\mathrm{H})}=\left(\Omega, \mathfrak{g}-\mathcal{T}_{\mathfrak{g}}^{(\mathrm{H})}\right)$, then it is a $\mathfrak{g}-\mathcal{T}_{\mathfrak{g}}^{(\mathrm{F})}$ space $\mathfrak{g}-\mathfrak{T}_{\mathfrak{g}}^{(\mathrm{F})}=\left(\Omega, \mathfrak{g}-\mathcal{T}_{\mathfrak{g}}^{(\mathrm{F})}\right)$.

A necessary and sufficient condition for a $\mathcal{T}_{\mathfrak{g}}$-space $\mathfrak{T}_{\mathfrak{g}}=\left(\Omega, \mathcal{T}_{\mathfrak{g}}\right)$ to be a $\mathfrak{g}-\mathcal{T}_{\mathfrak{g}}^{(\mathrm{R})}$ space $\mathfrak{g}-\mathfrak{T}_{\mathfrak{g}}^{(\mathrm{R})}=\left(\Omega, \mathfrak{g}-\mathcal{T}_{\mathfrak{g}}^{(\mathrm{R})}\right)$ may be given in terms of the $\mathfrak{g}$-operator op $\mathfrak{g}: \mathcal{P}(\Omega) \longrightarrow$ $\mathcal{P}(\Omega)$, a $\mathfrak{T}_{\mathfrak{g}}$-closed set $\mathcal{S}_{\mathfrak{g}} \subset \mathfrak{T}_{\mathfrak{g}}$, and $\mathcal{T}_{\mathfrak{g}}$-closed neighbourhood sets.

Theorem 3.4. A $\mathcal{T}_{\mathfrak{g}}$-space $\mathfrak{T}_{\mathfrak{g}}=\left(\Omega, \mathcal{T}_{\mathfrak{g}}\right)$ is said to be a $\mathfrak{g}-\mathcal{T}_{\mathfrak{g}}^{(\mathrm{R})}$-space $\mathfrak{g}-\mathfrak{T}_{\mathfrak{g}}^{(\mathrm{R})}=$ $\left(\Omega, \mathfrak{g}-\mathcal{T}_{\mathfrak{g}}^{(\mathrm{R})}\right)$ if and only if the following condition holds:

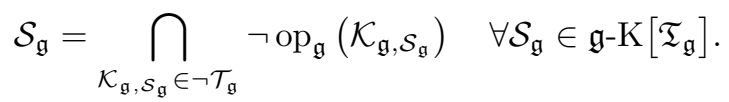

Proof. Necessity. Let the $\mathcal{T}_{\mathfrak{g}}$-space $\mathfrak{T}_{\mathfrak{g}}$ be a $\mathfrak{g}-\mathcal{T}_{\mathfrak{g}}^{(\mathrm{R})}$-space $\mathfrak{g}-\mathfrak{T}_{\mathfrak{g}}^{(\mathrm{R})}$. Then, for every $(\xi, \zeta) \in \mathfrak{g}-\mathrm{F}_{\mathrm{P}}[\sigma>0]$ and $\left(\mathcal{K}_{\mathfrak{g}, \xi}, \mathcal{K}_{\mathfrak{g}, \zeta}\right) \in \mathfrak{g}-\mathrm{F}_{\mathrm{K}}[\emptyset]$ such that $(\zeta, \xi) \notin\left(\mathcal{K}_{\mathfrak{g}, \xi}, \mathcal{K}_{\mathfrak{g}, \zeta}\right)$, there exists $\left(\mathcal{O}_{\mathfrak{g}, \xi}, \mathcal{O}_{\mathfrak{g}, \zeta}\right) \in \mathfrak{g}-\mathrm{F}_{\mathrm{O}}[\emptyset]$ such that:

$$
\begin{aligned}
{\left[\left(\neg \mathrm{op}_{\mathfrak{g}}\left(\mathcal{K}_{\mathfrak{g}, \xi}\right) \subset \mathrm{op}_{\mathfrak{g}}\left(\mathcal{O}_{\mathfrak{g}, \xi}\right)\right)\right.} & \left.\wedge\left(\zeta \in \mathrm{op}_{\mathfrak{g}}\left(\mathcal{O}_{\mathfrak{g}, \zeta}\right)\right)\right] \vee\left[\left(\neg \mathrm{op}_{\mathfrak{g}}\left(\mathcal{K}_{\mathfrak{g}, \zeta}\right)\right.\right. \\
& \left.\left.\subset \mathrm{op}_{\mathfrak{g}}\left(\mathcal{O}_{\mathfrak{g}, \zeta}\right)\right) \wedge\left(\xi \in \mathrm{op}_{\mathfrak{g}}\left(\mathcal{O}_{\mathfrak{g}, \xi}\right)\right)\right] .
\end{aligned}
$$

Consequently,

$$
\begin{aligned}
{\left[\left(\neg \mathrm{op}_{\mathfrak{g}}\left(\mathcal{K}_{\mathfrak{g}, \xi}\right) \subset \mathrm{op}_{\mathfrak{g}}\left(\mathcal{O}_{\mathfrak{g}, \xi}\right)\right)\right.} & \left.\wedge\left(\zeta \notin \complement\left(\mathrm{op}_{\mathfrak{g}}\left(\mathcal{O}_{\mathfrak{g}, \zeta}\right)\right)\right)\right] \vee\left[\left(\neg \mathrm{op}_{\mathfrak{g}}\left(\mathcal{K}_{\mathfrak{g}, \zeta}\right)\right.\right. \\
& \left.\left.\subset \mathrm{op}_{\mathfrak{g}}\left(\mathcal{O}_{\mathfrak{g}, \zeta}\right)\right) \wedge\left(\xi \notin \complement\left(\mathrm{op}_{\mathfrak{g}}\left(\mathcal{O}_{\mathfrak{g}, \xi}\right)\right)\right)\right] .
\end{aligned}
$$

But, for every $\lambda \in\{\xi, \zeta\}, \complement\left(\operatorname{op}_{\mathfrak{g}}\left(\mathcal{O}_{\mathfrak{g}, \lambda}\right)\right) \in \mathfrak{g}-\mathrm{K}\left[\mathfrak{T}_{\mathfrak{g}}\right]$ is a $\mathfrak{g}-\mathfrak{T}_{\mathfrak{g}}$-closed set. Con-

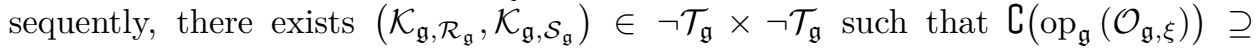
$\neg \mathrm{op}_{\mathfrak{g}}\left(\mathcal{K}_{\mathfrak{g}, \mathcal{R}_{\mathfrak{g}}}\right)$ and $\complement\left(\mathrm{op}_{\mathfrak{g}}\left(\mathcal{O}_{\mathfrak{g}, \zeta}\right)\right) \supseteq \neg \mathrm{op}_{\mathfrak{g}}\left(\mathcal{K}_{\mathfrak{g}, \mathcal{S}_{\mathfrak{g}}}\right)$. Therefore

$$
\begin{aligned}
& {\left[\left(\neg \mathrm{op}_{\mathfrak{g}}\left(\mathcal{K}_{\mathfrak{g}, \xi}\right) \subset \mathrm{op}_{\mathfrak{g}}\left(\mathcal{O}_{\mathfrak{g}, \xi}\right)\right) \wedge\left(\zeta \notin \neg \mathrm{op}_{\mathfrak{g}}\left(\mathcal{K}_{\mathfrak{g}, \mathcal{S}_{\mathfrak{g}}}\right)\right)\right] \vee\left[\left(\neg \mathrm{op}_{\mathfrak{g}}\left(\mathcal{K}_{\mathfrak{g}, \zeta}\right)\right.\right.} \\
& \left.\left.\subset \operatorname{op}_{\mathfrak{g}}\left(\mathcal{O}_{\mathfrak{g}, \zeta}\right)\right) \wedge\left(\xi \notin \neg \mathrm{op}_{\mathfrak{g}}\left(\mathcal{K}_{\mathfrak{g}, \mathcal{R}_{\mathfrak{g}}}\right)\right)\right] .
\end{aligned}
$$

By virtue of this logical statement, it consequently follows that

$$
\begin{aligned}
& {\left[\left(\neg \mathrm{op}_{\mathfrak{g}}\left(\mathcal{K}_{\mathfrak{g}, \xi}\right) \subset \mathrm{op}_{\mathfrak{g}}\left(\mathcal{O}_{\mathfrak{g}, \xi}\right)\right) \wedge\left(\neg \mathrm{op}_{\mathfrak{g}}\left(\mathcal{K}_{\mathfrak{g}, \xi}\right) \supseteq \neg \mathrm{op}_{\mathfrak{g}}\left(\mathcal{K}_{\mathfrak{g}, \mathcal{S}_{\mathfrak{g}}}\right)\right)\right]} \\
& \vee\left[\left(\neg \mathrm{op}_{\mathfrak{g}}\left(\mathcal{K}_{\mathfrak{g}, \zeta}\right) \subset \mathrm{op}_{\mathfrak{g}}\left(\mathcal{O}_{\mathfrak{g}, \zeta}\right)\right) \wedge\left(\neg \mathrm{op}_{\mathfrak{g}}\left(\mathcal{K}_{\mathfrak{g}, \xi}\right) \supseteq \neg \mathrm{op}_{\mathfrak{g}}\left(\mathcal{K}_{\mathfrak{g}, \mathcal{R}_{\mathfrak{g}}}\right)\right)\right],
\end{aligned}
$$


and, consequently,

$$
\begin{array}{r}
{\left[\neg \mathrm{op}_{\mathfrak{g}}\left(\mathcal{K}_{\mathfrak{g}, \mathcal{S}_{\mathfrak{g}}}\right) \subseteq \neg \mathrm{op}_{\mathfrak{g}}\left(\mathcal{K}_{\mathfrak{g}, \xi}\right) \subset \mathrm{op}_{\mathfrak{g}}\left(\mathcal{O}_{\mathfrak{g}, \xi}\right)\right]} \\
\vee\left[\neg \mathrm{op}_{\mathfrak{g}}\left(\mathcal{K}_{\mathfrak{g}, \mathcal{R}_{\mathfrak{g}}}\right) \subseteq \neg \mathrm{op}_{\mathfrak{g}}\left(\mathcal{K}_{\mathfrak{g}, \zeta}\right) \subset \mathrm{op}_{\mathfrak{g}}\left(\mathcal{O}_{\mathfrak{g}, \zeta}\right)\right],
\end{array}
$$

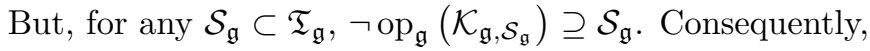

$$
\begin{aligned}
& {\left[\mathcal{S}_{\mathfrak{g}} \subseteq \neg \mathrm{op}_{\mathfrak{g}}\left(\mathcal{K}_{\mathfrak{g}, \mathcal{S}_{\mathfrak{g}}}\right) \subseteq \neg \mathrm{op}_{\mathfrak{g}}\left(\mathcal{K}_{\mathfrak{g}, \xi}\right) \subset \mathrm{op}_{\mathfrak{g}}\left(\mathcal{O}_{\mathfrak{g}, \xi}\right)\right]} \\
& \vee\left[\mathcal{R}_{\mathfrak{g}} \subseteq \neg \mathrm{op}_{\mathfrak{g}}\left(\mathcal{K}_{\mathfrak{g}, \mathcal{R}_{\mathfrak{g}}}\right) \subseteq \neg \mathrm{op}_{\mathfrak{g}}\left(\mathcal{K}_{\mathfrak{g}, \zeta}\right) \subset \mathrm{op}_{\mathfrak{g}}\left(\mathcal{O}_{\mathfrak{g}, \zeta}\right)\right],
\end{aligned}
$$

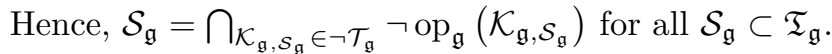

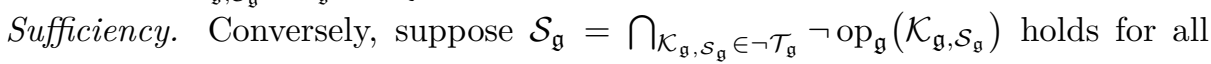
$\mathcal{S}_{\mathfrak{g}} \subset \mathfrak{T}_{\mathfrak{g}}$, let $\xi \notin \mathcal{S}_{\mathfrak{g}}$. Then, $\mathcal{S}_{\mathfrak{g}} \subseteq \neg \mathrm{op}_{\mathfrak{g}}\left(\mathcal{K}_{\mathfrak{g}, \mathcal{S}_{\mathfrak{g}}}\right)$ for every $\mathcal{T}_{\mathfrak{g}}$-closed neighbourhood set $\mathcal{K}_{\mathfrak{g}, \mathcal{S}_{\mathfrak{g}}} \in \neg \mathcal{T}_{\mathfrak{g}}$ satisfying $\mathcal{S}_{\mathfrak{g}} \subseteq \mathcal{K}_{\mathfrak{g}, \mathcal{S}_{\mathfrak{g}}}$. Therefore, there exists a $\mathcal{T}_{\mathfrak{g}}$-closed neighbourhood set $\mathcal{K}_{\mathfrak{g}, \mathcal{S}_{\mathfrak{g}}} \in \neg \mathcal{T}_{\mathfrak{g}}$ such that $\xi \notin \mathcal{K}_{\mathfrak{g}, \mathcal{S}_{\mathfrak{g}}}$. But, since $\mathcal{K}_{\mathfrak{g}, \mathcal{S}_{\mathfrak{g}}} \in \neg \mathcal{T}_{\mathfrak{g}}$ is a $\mathcal{T}_{\mathfrak{g}}$-closed neighbourhood set, there exists a $\mathcal{T}_{\mathfrak{g}}$-open set $\mathcal{O}_{\mathfrak{g}, \mathcal{S}_{\mathfrak{g}}} \in \mathcal{T}_{\mathfrak{g}}$ such that $\mathcal{S}_{\mathfrak{g}} \subset \mathrm{op}_{\mathfrak{g}}\left(\mathcal{O}_{\mathfrak{g}, \mathcal{S}_{\mathfrak{g}}}\right) \subset \neg \mathrm{op}_{\mathfrak{g}}\left(\mathcal{K}_{\mathfrak{g}, \mathcal{S}_{\mathfrak{g}}}\right)$, and $\complement\left(\mathcal{S}_{\mathfrak{g}}\right) \supset \complement\left(\operatorname{op}_{\mathfrak{g}}\left(\mathcal{O}_{\mathfrak{g}, \mathcal{S}_{\mathfrak{g}}}\right)\right) \supset \complement\left(\neg \mathrm{op}_{\mathfrak{g}}\left(\mathcal{K}_{\mathfrak{g}, \mathcal{S}_{\mathfrak{g}}}\right)\right)$. Because $\xi \in \complement\left(\neg \operatorname{op}_{\mathfrak{g}}\left(\mathcal{K}_{\mathfrak{g}, \mathcal{S}_{\mathfrak{g}}}\right)\right)$ and $\xi \in \complement\left(\operatorname{op}_{\mathfrak{g}}\left(\mathcal{O}_{\mathfrak{g}, \mathcal{S}_{\mathfrak{g}}}\right)\right)$, it follows that op $\operatorname{op}_{\mathfrak{g}}\left(\mathcal{O}_{\mathfrak{g}, \mathcal{S}_{\mathfrak{g}}}\right) \cap$ $\complement\left(\neg \mathrm{op}_{\mathfrak{g}}\left(\mathcal{K}_{\mathfrak{g}, \mathcal{S}_{\mathfrak{g}}}\right)\right)=\emptyset$ and $\complement\left(\mathrm{op}_{\mathfrak{g}}\left(\mathcal{O}_{\mathfrak{g}, \mathcal{S}_{\mathfrak{g}}}\right)\right) \cap \neg \mathrm{op}_{\mathfrak{g}}\left(\mathcal{K}_{\mathfrak{g}, \mathcal{S}_{\mathfrak{g}}}\right)=\emptyset$, respectively. In other words, for every $(\xi, \zeta) \in \mathfrak{g}-\mathrm{F}_{\mathrm{P}}[\sigma>0]$ and $\left(\mathcal{K}_{\mathfrak{g}, \xi}, \mathcal{K}_{\mathfrak{g}, \zeta}\right) \in \mathfrak{g}-\mathrm{F}_{\mathrm{K}}[\emptyset]$ such that $(\zeta, \xi) \notin\left(\mathcal{K}_{\mathfrak{g}, \xi}, \mathcal{K}_{\mathfrak{g}, \zeta}\right)$, there exists $\left(\mathcal{O}_{\mathfrak{g}, \xi}, \mathcal{O}_{\mathfrak{g}, \zeta}\right) \in \mathfrak{g}^{-\mathrm{F}_{\mathrm{O}}}[\emptyset]$ such that:

$$
\begin{aligned}
{\left[\left(\neg \mathrm{op}_{\mathfrak{g}}\left(\mathcal{K}_{\mathfrak{g}, \xi}\right) \subset \mathrm{op}_{\mathfrak{g}}\left(\mathcal{O}_{\mathfrak{g}, \xi}\right)\right)\right.} & \left.\wedge\left(\zeta \in \mathrm{op}_{\mathfrak{g}}\left(\mathcal{O}_{\mathfrak{g}, \zeta}\right)\right)\right] \vee\left[\left(\neg \mathrm{op}_{\mathfrak{g}}\left(\mathcal{K}_{\mathfrak{g}, \zeta}\right)\right.\right. \\
& \left.\left.\subset \mathrm{op}_{\mathfrak{g}}\left(\mathcal{O}_{\mathfrak{g}, \zeta}\right)\right) \wedge\left(\xi \in \mathrm{op}_{\mathfrak{g}}\left(\mathcal{O}_{\mathfrak{g}, \xi}\right)\right)\right] .
\end{aligned}
$$

Therefore, $\mathfrak{T}_{\mathfrak{g}}=\left(\Omega, \mathcal{T}_{\mathfrak{g}}\right)$ is a $\mathfrak{g}-\mathcal{T}_{\mathfrak{g}}^{(\mathrm{R})}$-space $\mathfrak{g}-\mathfrak{T}_{\mathfrak{g}}^{(\mathrm{R})}=\left(\Omega, \mathfrak{g}-\mathcal{T}_{\mathfrak{g}}^{(\mathrm{R})}\right)$; this completes the proof of the theorem.

Proposition 3. If $\mathfrak{T}_{\mathfrak{g}}=\left(\Omega, \mathcal{T}_{\mathfrak{g}}\right)$ is a $\mathfrak{g}-\mathcal{T}_{\mathfrak{g}}^{(\mathrm{R})}$-space $\mathfrak{g}-\mathfrak{T}_{\mathfrak{g}}^{(\mathrm{R})}=\left(\Omega, \mathfrak{g}-\mathcal{T}_{\mathfrak{g}}^{(\mathrm{R})}\right)$, then it is a $\mathfrak{g}-\mathcal{T}_{\mathfrak{g}}^{(\mathrm{H})}$-space $\mathfrak{g}-\mathfrak{T}_{\mathfrak{g}}^{(\mathrm{H})}=\left(\Omega, \mathfrak{g}-\mathcal{T}_{\mathfrak{g}}^{(\mathrm{H})}\right)$.

Proof. Let $\mathfrak{T}_{\mathfrak{g}}=\left(\Omega, \mathcal{T}_{\mathfrak{g}}\right)$ be a $\mathfrak{g}-\mathcal{T}_{\mathfrak{g}}^{(\mathrm{R})}$-space $\mathfrak{g}-\mathfrak{T}_{\mathfrak{g}}^{(\mathrm{R})}=\left(\Omega, \mathfrak{g}-\mathcal{T}_{\mathfrak{g}}^{(\mathrm{R})}\right)$. Then, for every $(\xi, \zeta) \in \mathfrak{g}-\mathrm{F}_{\mathrm{P}}[\sigma>0]$ and $\left(\mathcal{K}_{\mathfrak{g}, \xi}, \mathcal{K}_{\mathfrak{g}, \zeta}\right) \in \mathfrak{g}-\mathrm{F}_{\mathrm{K}}[\emptyset]$ such that $(\zeta, \xi) \notin\left(\mathcal{K}_{\mathfrak{g}, \xi}, \mathcal{K}_{\mathfrak{g}, \zeta}\right)$, there exists $\left(\mathcal{O}_{\mathfrak{g}, \xi}, \mathcal{O}_{\mathfrak{g}, \zeta}\right) \in \mathfrak{g}-\mathrm{F}_{\mathrm{O}}[\emptyset]$ such that:

$$
\begin{aligned}
{\left[\left(\neg \mathrm{op}_{\mathfrak{g}}\left(\mathcal{K}_{\mathfrak{g}, \xi}\right) \subset \mathrm{op}_{\mathfrak{g}}\left(\mathcal{O}_{\mathfrak{g}, \xi}\right)\right)\right.} & \left.\wedge\left(\zeta \in \mathrm{op}_{\mathfrak{g}}\left(\mathcal{O}_{\mathfrak{g}, \zeta}\right)\right)\right] \vee\left[\left(\neg \mathrm{op}_{\mathfrak{g}}\left(\mathcal{K}_{\mathfrak{g}, \zeta}\right)\right.\right. \\
& \left.\left.\subset \mathrm{op}_{\mathfrak{g}}\left(\mathcal{O}_{\mathfrak{g}, \zeta}\right)\right) \wedge\left(\xi \in \mathrm{op}_{\mathfrak{g}}\left(\mathcal{O}_{\mathfrak{g}, \xi}\right)\right)\right] .
\end{aligned}
$$

Set $\mathrm{Q}(\xi)=\left(\neg \mathrm{op}_{\mathfrak{g}}\left(\mathcal{K}_{\mathfrak{g}, \xi}\right) \subset \mathrm{op}_{\mathfrak{g}}\left(\mathcal{O}_{\mathfrak{g}, \xi}\right)\right), \mathrm{R}(\zeta)=\left(\zeta \in \mathrm{op}_{\mathfrak{g}}\left(\mathcal{O}_{\mathfrak{g}, \zeta}\right)\right)$, and $\mathrm{P}(\xi, \zeta)=$ $\mathrm{Q}(\xi) \wedge \mathrm{R}(\zeta)$. Then, the above logical statement is equivalent to $\mathrm{P}(\xi, \zeta) \vee \mathrm{P}(\zeta, \xi)$. But since $\lambda \in \neg \mathrm{op}_{\mathfrak{g}}\left(\mathcal{K}_{\mathfrak{g}, \lambda}\right)$ for every $\lambda\{\xi, \zeta\}$, it consequently follows that $\mathrm{R}(\lambda) \longleftarrow$ $\mathrm{Q}(\lambda)$ for every $\lambda\{\xi, \zeta\}$. Therefore $\mathrm{R}(\xi) \wedge \mathrm{R}(\zeta) \longleftarrow \mathrm{P}(\xi, \zeta)$. Because associativity with respect to $\wedge$ holds, it then follows that

$$
\mathrm{P}(\xi, \zeta) \vee \mathrm{P}(\zeta, \xi) \longrightarrow[\mathrm{R}(\xi) \wedge \mathrm{R}(\zeta)] \vee[\mathrm{R}(\zeta) \wedge \mathrm{R}(\xi)]=\mathrm{R}(\xi) \wedge \mathrm{R}(\zeta)
$$

Hence, for every $(\xi, \zeta) \in \mathfrak{g}-\mathrm{F}_{\mathrm{P}}[\sigma>0]$, there exists $\left(\mathcal{O}_{\mathfrak{g}, \xi}, \mathcal{O}_{\mathfrak{g}, \zeta}\right) \in \mathfrak{g}-\mathrm{F}_{\mathrm{O}}[\emptyset]$ such that:

$$
\left[\xi \in \mathrm{op}_{\mathfrak{g}}\left(\mathcal{O}_{\mathfrak{g}, \xi}\right)\right] \wedge\left[\zeta \in \mathrm{op}_{\mathfrak{g}}\left(\mathcal{O}_{\mathfrak{g}, \zeta}\right)\right] .
$$

This proves that, if $\mathfrak{T}_{\mathfrak{g}}=\left(\Omega, \mathcal{T}_{\mathfrak{g}}\right)$ is a $\mathfrak{g}-\mathcal{T}_{\mathfrak{g}}^{(\mathrm{R})}$-space $\mathfrak{g}-\mathfrak{T}_{\mathfrak{g}}^{(\mathrm{R})}=\left(\Omega, \mathfrak{g}-\mathcal{T}_{\mathfrak{g}}^{(\mathrm{R})}\right)$, then it is a $\mathfrak{g}-\mathcal{T}_{\mathfrak{g}}^{(\mathrm{H})}$-space $\mathfrak{g}-\mathfrak{T}_{\mathfrak{g}}^{(\mathrm{H})}=\left(\Omega, \mathfrak{g}-\mathcal{T}_{\mathfrak{g}}^{(\mathrm{H})}\right)$. 
A necessary and sufficient condition for a $\mathcal{T}_{\mathfrak{g}}$-space $\mathfrak{T}_{\mathfrak{g}}=\left(\Omega, \mathcal{T}_{\mathfrak{g}}\right)$ to be a $\mathfrak{g}-\mathcal{T}_{\mathfrak{g}}^{(\mathrm{N})}$ space $\mathfrak{g}-\mathfrak{T}_{\mathfrak{g}}^{(\mathrm{N})}=\left(\Omega, \mathfrak{g}-\mathcal{T}_{\mathfrak{g}}^{(\mathrm{N})}\right)$ may be given in terms of the $\mathfrak{g}$-operator op $\mathfrak{g}: \mathcal{P}(\Omega) \longrightarrow$ $\mathcal{P}(\Omega)$, a $\mathfrak{T}_{\mathfrak{g}}$-closed set $\mathcal{S}_{\mathfrak{g}} \subset \mathfrak{T}_{\mathfrak{g}}, \mathcal{T}_{\mathfrak{g}}$-open sets, and a $\mathcal{T}_{\mathfrak{g}}$-closed set.

Theorem 3.5. A $\mathcal{T}_{\mathfrak{g}}$-space $\mathfrak{T}_{\mathfrak{g}}=\left(\Omega, \mathcal{T}_{\mathfrak{g}}\right)$ is said to be a $\mathfrak{g}-\mathcal{T}_{\mathfrak{g}}^{(\mathrm{N})}$-space $\mathfrak{g}-\mathfrak{T}_{\mathfrak{g}}^{(\mathrm{N})}=$ $\left(\Omega, \mathfrak{g}-\mathcal{T}_{\mathfrak{g}}^{(\mathrm{N})}\right)$ if and only if the following condition holds:

$$
\mathcal{S}_{\mathfrak{g}} \subset \operatorname{op}_{\mathfrak{g}}\left(\hat{\mathcal{O}}_{\mathfrak{g}, \mathcal{S}_{\mathfrak{g}}}\right) \subset \neg \mathrm{op}_{\mathfrak{g}}\left(\hat{\mathcal{K}}_{\mathfrak{g}, \mathcal{S}_{\mathfrak{g}}}\right) \subset \mathrm{op}_{\mathfrak{g}}\left(\mathcal{O}_{\mathfrak{g}, \mathcal{S}_{\mathfrak{g}}}\right) \quad \forall \mathcal{S}_{\mathfrak{g}} \in \mathfrak{g}-\mathrm{K}\left[\mathfrak{T}_{\mathfrak{g}}\right] .
$$

Proof. Necessity. Let the $\mathcal{T}_{\mathfrak{g}}$-space $\mathfrak{T}_{\mathfrak{g}}$ be a $\mathfrak{g}-\mathcal{T}_{\mathfrak{g}}^{(\mathrm{N})}$-space $\mathfrak{g}$ - $\mathfrak{T}_{\mathfrak{g}}^{(\mathrm{N})}$ and, let $\mathcal{S}_{\mathfrak{g}} \in$ $\mathfrak{g}-\mathrm{K}\left[\mathfrak{T}_{\mathfrak{g}}\right]$ and $\mathcal{O}_{\mathfrak{g}, \mathcal{S}_{\mathfrak{g}}} \in \mathcal{T}_{\mathfrak{g}}$, respectively, be a $\mathfrak{g}-\mathfrak{T}_{\mathfrak{g}}$-closed set and a $\mathcal{T}_{\mathfrak{g}}$-open neighbourhood set of $\mathcal{S}_{\mathfrak{g}}$. Then, $\mathfrak{T}_{\mathfrak{g}}$ is a $\mathfrak{g}-\mathcal{T}_{\mathfrak{g}}^{(\mathrm{N})}$-space $\mathfrak{g}-\mathfrak{T}_{\mathfrak{g}}^{(\mathrm{N})}$ implies that, for every $\left(\mathcal{K}_{\mathfrak{g}, \xi}, \mathcal{K}_{\mathfrak{g}, \zeta}\right) \in \mathfrak{g}-\mathrm{F}_{K}[\emptyset]$, there exists $\left(\mathcal{O}_{\mathfrak{g}, \xi}, \mathcal{O}_{\mathfrak{g}, \zeta}\right) \in \mathfrak{g}-\mathrm{F}_{\mathrm{O}}[\emptyset]$ such that:

$$
\left[\operatorname{op}_{\mathfrak{g}}\left(\mathcal{O}_{\mathfrak{g}, \xi}\right) \supset \neg \mathrm{op}_{\mathfrak{g}}\left(\mathcal{K}_{\mathfrak{g}, \xi}\right)\right] \wedge\left[\operatorname{op}_{\mathfrak{g}}\left(\mathcal{O}_{\mathfrak{g}, \zeta}\right) \supset \neg \mathrm{op}_{\mathfrak{g}}\left(\mathcal{K}_{\mathfrak{g}, \zeta}\right)\right] .
$$

Clearly, $\complement\left(\operatorname{op}_{\mathfrak{g}}\left(\mathcal{O}_{\mathfrak{g}, \xi}\right)\right) \cap \neg \mathrm{op}_{\mathfrak{g}}\left(\mathcal{K}_{\mathfrak{g}, \xi}\right)=\emptyset$ for any $\xi \in \mathfrak{T}_{\mathfrak{g}}$. The relation $\mathcal{S}_{\mathfrak{g}} \in \mathfrak{g}-\mathrm{K}\left[\mathfrak{T}_{\mathfrak{g}}\right]$ implies that there exists a $\hat{\mathcal{K}}_{\mathfrak{g}, \xi} \in \neg \mathcal{T}_{\mathfrak{g}}$ such that $\mathcal{S}_{\mathfrak{g}} \supseteq \neg \mathrm{op}_{\mathfrak{g}}\left(\hat{\mathcal{K}}_{\mathfrak{g}, \xi}\right)$ and, $\mathcal{O}_{\mathfrak{g}, \mathcal{S}_{\mathfrak{g}}} \in \mathcal{T}_{\mathfrak{g}}$ is a $\mathcal{T}_{\mathfrak{g}}$-open neighbourhood set of $\mathcal{S}_{\mathfrak{g}}$ implies that there exists $\left(\hat{\mathcal{O}}_{\mathfrak{g}, \mathcal{S}_{\mathfrak{g}}}, \hat{\mathcal{K}}_{\mathfrak{g}, \mathcal{S}_{\mathfrak{g}}}\right) \in$ $\mathcal{T}_{\mathfrak{g}} \times \neg \mathcal{T}_{\mathfrak{g}}$ such that $\mathcal{O}_{\mathfrak{g}, \mathcal{S}_{\mathfrak{g}}} \subseteq \operatorname{op}_{\mathfrak{g}}\left(\hat{\mathcal{O}}_{\mathfrak{g}, \mathcal{S}_{\mathfrak{g}}}\right) \subset \neg \mathrm{op}_{\mathfrak{g}}\left(\hat{\mathcal{K}}_{\mathfrak{g}, \mathcal{S}_{\mathfrak{g}}}\right)$. But, $\mathcal{S}_{\mathfrak{g}} \subset \mathcal{O}_{\mathfrak{g}, \mathcal{S}_{\mathfrak{g}}}$ and, for some $\hat{\mathcal{O}}_{\mathfrak{g}, \xi} \in \neg \mathcal{T}_{\mathfrak{g}}$, the relation $\neg \mathrm{op}_{\mathfrak{g}}\left(\hat{\mathcal{K}}_{\mathfrak{g}, \mathcal{S}_{\mathfrak{g}}}\right) \subset \mathrm{op}_{\mathfrak{g}}\left(\hat{\mathcal{O}}_{\mathfrak{g}, \xi}\right)$ holds in a $\mathfrak{g}-\mathcal{T}_{\mathfrak{g}}^{(\mathrm{N})}$-space $\mathfrak{g}-\mathfrak{T}_{\mathfrak{g}}^{(\mathrm{N})}$. Hence,

$$
\neg \mathrm{op}_{\mathfrak{g}}\left(\hat{\mathcal{K}}_{\mathfrak{g}, \xi}\right) \subseteq \mathcal{S}_{\mathfrak{g}} \subset \mathrm{op}_{\mathfrak{g}}\left(\hat{\mathcal{O}}_{\mathfrak{g}, \mathcal{S}_{\mathfrak{g}}}\right) \subset \neg \mathrm{op}_{\mathfrak{g}}\left(\hat{\mathcal{K}}_{\mathfrak{g}, \mathcal{S}_{\mathfrak{g}}}\right) \subset \mathrm{op}_{\mathfrak{g}}\left(\hat{\mathcal{O}}_{\mathfrak{g}, \xi}\right)
$$

for all $\mathcal{S}_{\mathfrak{g}} \in \mathfrak{g}-\mathrm{K}\left[\mathfrak{T}_{\mathfrak{g}}\right]$. At this stage, it suffices to set $\hat{\mathcal{O}}_{\mathfrak{g}, \xi} \subseteq \mathcal{O}_{\mathfrak{g}, \mathcal{S}_{\mathfrak{g}}}$ and the result follows.

Sufficiency. Conversely, suppose the following relation holds:

$$
\mathcal{S}_{\mathfrak{g}} \subset \operatorname{op}_{\mathfrak{g}}\left(\hat{\mathcal{O}}_{\mathfrak{g}, \mathcal{S}_{\mathfrak{g}}}\right) \subset \neg \mathrm{op}_{\mathfrak{g}}\left(\hat{\mathcal{K}}_{\mathfrak{g}, \mathcal{S}_{\mathfrak{g}}}\right) \subset \mathrm{op}_{\mathfrak{g}}\left(\mathcal{O}_{\mathfrak{g}, \mathcal{S}_{\mathfrak{g}}}\right) \quad \forall \mathcal{S}_{\mathfrak{g}} \in \mathfrak{g}-\mathrm{K}\left[\mathfrak{T}_{\mathfrak{g}}\right] .
$$

Then, its complementary reads

$$
\complement\left(\mathcal{S}_{\mathfrak{g}}\right) \supset \complement\left(\mathrm{op}_{\mathfrak{g}}\left(\hat{\mathcal{O}}_{\mathfrak{g}, \mathcal{S}_{\mathfrak{g}}}\right)\right) \supset \complement\left(\neg \mathrm{op}_{\mathfrak{g}}\left(\hat{\mathcal{K}}_{\mathfrak{g}, \mathcal{S}_{\mathfrak{g}}}\right)\right) \supset \complement\left(\mathrm{op}_{\mathfrak{g}}\left(\mathcal{O}_{\mathfrak{g}, \mathcal{S}_{\mathfrak{g}}}\right)\right),
$$

where $\complement\left(\mathcal{S}_{\mathfrak{g}}\right), \complement\left(\neg \operatorname{op}_{\mathfrak{g}}\left(\hat{\mathcal{K}}_{\mathfrak{g}, \mathcal{S}_{\mathfrak{g}}}\right)\right) \in \mathfrak{g}$-O $\left[\mathfrak{T}_{\mathfrak{g}}\right]$ are $\mathfrak{g}$ - $\mathfrak{T}_{\mathfrak{g}}$-open sets and, $\complement\left(\operatorname{op}_{\mathfrak{g}}\left(\hat{\mathcal{O}}_{\mathfrak{g}, \mathcal{S}_{\mathfrak{g}}}\right)\right)$, $\complement\left(\operatorname{op}_{\mathfrak{g}}\left(\mathcal{O}_{\mathfrak{g}, \mathcal{S}_{\mathfrak{g}}}\right)\right) \in \mathfrak{g}-\mathrm{K}\left[\mathfrak{T}_{\mathfrak{g}}\right]$ are $\mathfrak{g}-\mathfrak{T}_{\mathfrak{g}}$-closed sets. Thus, $\mathcal{S}_{\mathfrak{g}} \cap \complement\left(\operatorname{op}_{\mathfrak{g}}\left(\mathcal{O}_{\mathfrak{g}, \mathcal{S}_{\mathfrak{g}}}\right)\right)=\emptyset$ for any $\mathcal{S}_{\mathfrak{g}} \in \mathfrak{g}-\mathrm{K}\left[\mathfrak{T}_{\mathfrak{g}}\right]$. But since the relation $\mathcal{S}_{\mathfrak{g}} \supseteq \neg \mathrm{op}_{\mathfrak{g}}\left(\mathcal{K}_{\mathfrak{g}, \xi}\right)$ holds for some $\mathcal{K}_{\mathfrak{g}, \xi} \in$ $\neg \mathcal{T}_{\mathfrak{g}}$, it consequently follows that $\complement\left(\operatorname{op}_{\mathfrak{g}}\left(\mathcal{O}_{\mathfrak{g}, \mathcal{S}_{\mathfrak{g}}}\right)\right) \subset \complement\left(\mathcal{S}_{\mathfrak{g}}\right) \subseteq \complement\left(\neg \mathrm{op}_{\mathfrak{g}}\left(\mathcal{K}_{\mathfrak{g}, \xi}\right)\right)$ which, in turn, implies $\operatorname{op}_{\mathfrak{g}}\left(\mathcal{O}_{\mathfrak{g}, \mathcal{S}_{\mathfrak{g}}}\right) \supset \mathcal{S}_{\mathfrak{g}} \supseteq \neg \operatorname{op}_{\mathfrak{g}}\left(\mathcal{K}_{\mathfrak{g}, \xi}\right)$. Thus, for every $\left(\mathcal{K}_{\mathfrak{g}, \xi}, \mathcal{K}_{\mathfrak{g}, \zeta}\right) \in$ $\mathfrak{g}-\mathrm{F}_{\mathrm{K}}[\emptyset]$, there exists $\left(\mathcal{O}_{\mathfrak{g}, \xi}, \mathcal{O}_{\mathfrak{g}, \zeta}\right) \in \mathfrak{g}-\mathrm{F}_{\mathrm{O}}[\emptyset]$ such that:

$$
\left[\operatorname{op}_{\mathfrak{g}}\left(\mathcal{O}_{\mathfrak{g}, \xi}\right) \supset \neg \mathrm{op}_{\mathfrak{g}}\left(\mathcal{K}_{\mathfrak{g}, \xi}\right)\right] \wedge\left[\operatorname{op}_{\mathfrak{g}}\left(\mathcal{O}_{\mathfrak{g}, \zeta}\right) \supset \neg \mathrm{op}_{\mathfrak{g}}\left(\mathcal{K}_{\mathfrak{g}, \zeta}\right)\right] .
$$

Therefore, $\mathfrak{T}_{\mathfrak{g}}=\left(\Omega, \mathcal{T}_{\mathfrak{g}}\right)$ is a $\mathfrak{g}-\mathcal{T}_{\mathfrak{g}}^{(\mathrm{N})}$-space $\mathfrak{g}-\mathfrak{T}_{\mathfrak{g}}^{(\mathrm{N})}=\left(\Omega, \mathfrak{g}-\mathcal{T}_{\mathfrak{g}}^{(\mathrm{N})}\right)$; this completes the proof of the theorem.

Proposition 4. If $\mathfrak{T}_{\mathfrak{g}}=\left(\Omega, \mathcal{T}_{\mathfrak{g}}\right)$ is a $\mathfrak{g}-\mathcal{T}_{\mathfrak{g}}^{(\mathrm{N})}$-space $\mathfrak{g}-\mathfrak{T}_{\mathfrak{g}}^{(\mathrm{N})}=\left(\Omega, \mathfrak{g}-\mathcal{T}_{\mathfrak{g}}^{(\mathrm{N})}\right)$, then it is a $\mathfrak{g}-\mathcal{T}_{\mathfrak{g}}^{(\mathrm{R})}$-space $\mathfrak{g}-\mathfrak{T}_{\mathfrak{g}}^{(\mathrm{R})}=\left(\Omega, \mathfrak{g}-\mathcal{T}_{\mathfrak{g}}^{(\mathrm{R})}\right)$.

Proof. Let $\mathfrak{T}_{\mathfrak{g}}=\left(\Omega, \mathcal{T}_{\mathfrak{g}}\right)$ be a $\mathfrak{g}-\mathcal{T}_{\mathfrak{g}}^{(\mathrm{N})}$-space $\mathfrak{g}-\mathfrak{T}_{\mathfrak{g}}^{(\mathrm{N})}=\left(\Omega, \mathfrak{g}-\mathcal{T}_{\mathfrak{g}}^{(\mathrm{N})}\right)$. Then, for every $\left(\mathcal{K}_{\mathfrak{g}, \xi}, \mathcal{K}_{\mathfrak{g}, \zeta}\right) \in \mathfrak{g}-\mathrm{F}_{\mathrm{K}}[\emptyset]$, there exists $\left(\mathcal{O}_{\mathfrak{g}, \xi}, \mathcal{O}_{\mathfrak{g}, \zeta}\right) \in \mathfrak{g}-\mathrm{F}_{\mathrm{O}}[\emptyset]$ such that:

$$
\left[\mathrm{op}_{\mathfrak{g}}\left(\mathcal{O}_{\mathfrak{g}, \xi}\right) \supset \neg \mathrm{op}_{\mathfrak{g}}\left(\mathcal{K}_{\mathfrak{g}, \xi}\right)\right] \wedge\left[\mathrm{op}_{\mathfrak{g}}\left(\mathcal{O}_{\mathfrak{g}, \zeta}\right) \supset \neg \mathrm{op}_{\mathfrak{g}}\left(\mathcal{K}_{\mathfrak{g}, \zeta}\right)\right] .
$$


Set $\mathrm{Q}(\xi)=\left(\neg \mathrm{op}_{\mathfrak{g}}\left(\mathcal{K}_{\mathfrak{g}, \xi}\right) \subset \mathrm{op}_{\mathfrak{g}}\left(\mathcal{O}_{\mathfrak{g}, \xi}\right)\right)$ and $\mathrm{R}(\xi)=\left(\xi \in \mathrm{op}_{\mathfrak{g}}\left(\mathcal{O}_{\mathfrak{g}, \xi}\right)\right)$ so that the above logical statement now reads $\mathrm{Q}(\xi) \wedge \mathrm{Q}(\zeta)$. Then, since $\left(\mathcal{K}_{\mathfrak{g}, \xi}, \mathcal{K}_{\mathfrak{g}, \zeta}\right) \in \mathfrak{g}-\mathrm{F}_{\mathrm{K}}[\emptyset]$, $\mathrm{Q}(\xi) \wedge \mathrm{R}(\zeta) \longleftarrow \mathrm{Q}(\xi)$ and $\mathrm{Q}(\zeta) \wedge \mathrm{R}(\xi) \longleftarrow \mathrm{Q}(\zeta)$ hold. Consequently,

$$
[\mathrm{Q}(\xi) \wedge \mathrm{R}(\zeta)] \wedge[\mathrm{Q}(\zeta) \wedge \mathrm{R}(\xi)] \longleftarrow \mathrm{Q}(\xi) \wedge \mathrm{Q}(\zeta)
$$

But

$$
[\mathrm{Q}(\xi) \wedge \mathrm{R}(\zeta)] \vee[\mathrm{Q}(\zeta) \wedge \mathrm{R}(\xi)] \longleftarrow[\mathrm{Q}(\xi) \wedge \mathrm{R}(\zeta)] \wedge[\mathrm{Q}(\zeta) \wedge \mathrm{R}(\xi)]
$$

and, therefore,

$$
[\mathrm{Q}(\xi) \wedge \mathrm{R}(\zeta)] \vee[\mathrm{Q}(\zeta) \wedge \mathrm{R}(\xi)] \longleftarrow \mathrm{Q}(\xi) \wedge \mathrm{Q}(\zeta)
$$

Thus, for every $(\xi, \zeta) \in \mathfrak{g}-\mathrm{F}_{\mathrm{P}}[\sigma>0]$ and $\left(\mathcal{K}_{\mathfrak{g}, \xi}, \mathcal{K}_{\mathfrak{g}, \zeta}\right) \in \mathfrak{g}-\mathrm{F}_{\mathrm{K}}[\emptyset]$ such that $(\zeta, \xi) \notin$ $\left(\mathcal{K}_{\mathfrak{g}, \xi}, \mathcal{K}_{\mathfrak{g}, \zeta}\right)$, there exists $\left(\mathcal{O}_{\mathfrak{g}, \xi}, \mathcal{O}_{\mathfrak{g}, \zeta}\right) \in \mathfrak{g}-\mathrm{F}_{\mathrm{O}}[\emptyset]$ such that:

$$
\begin{aligned}
{\left[\left(\neg \mathrm{op}_{\mathfrak{g}}\left(\mathcal{K}_{\mathfrak{g}, \xi}\right) \subset \mathrm{op}_{\mathfrak{g}}\left(\mathcal{O}_{\mathfrak{g}, \xi}\right)\right)\right.} & \left.\wedge\left(\zeta \in \mathrm{op}_{\mathfrak{g}}\left(\mathcal{O}_{\mathfrak{g}, \zeta}\right)\right)\right] \vee\left[\left(\neg \mathrm{op}_{\mathfrak{g}}\left(\mathcal{K}_{\mathfrak{g}, \zeta}\right)\right.\right. \\
& \left.\left.\subset \mathrm{op}_{\mathfrak{g}}\left(\mathcal{O}_{\mathfrak{g}, \zeta}\right)\right) \wedge\left(\xi \in \mathrm{op}_{\mathfrak{g}}\left(\mathcal{O}_{\mathfrak{g}, \xi}\right)\right)\right] .
\end{aligned}
$$

This proves that, if $\mathfrak{T}_{\mathfrak{g}}=\left(\Omega, \mathcal{T}_{\mathfrak{g}}\right)$ is a $\mathfrak{g}-\mathcal{T}_{\mathfrak{g}}^{(\mathrm{N})}$-space $\mathfrak{g}-\mathfrak{T}_{\mathfrak{g}}^{(\mathrm{N})}=\left(\Omega, \mathfrak{g}-\mathcal{T}_{\mathfrak{g}}^{(\mathrm{N})}\right)$, then it is a $\mathfrak{g}-\mathcal{T}_{\mathfrak{g}}^{(\mathrm{R})}$-space $\mathfrak{g}-\mathfrak{T}_{\mathfrak{g}}^{(\mathrm{R})}=\left(\Omega, \mathfrak{g}-\mathcal{T}_{\mathfrak{g}}^{(\mathrm{R})}\right)$.

By virtue of the above propositions, every $\mathfrak{g}-\mathcal{T}_{\mathfrak{g}}^{(\mathrm{H})}$-space is a $\mathfrak{g}-\mathcal{T}_{\mathfrak{g}}^{(\mathrm{F})}$-space, and hence, a $\mathfrak{g}-\mathcal{T}_{\mathfrak{g}}^{(\mathrm{K})}$-space. Also, every $\mathfrak{g}-\mathcal{T}_{\mathfrak{g}}^{(\mathrm{N})}$-space is a $\mathfrak{g}-\mathcal{T}_{\mathfrak{g}}^{(\mathrm{R})}$-space, and hence, a $\mathfrak{g}-\mathcal{T}_{\mathfrak{g}}^{(\mathrm{H})}$-space. But, the converse of both statements are untrue, and thus, the corollary follows. If $\left\langle\mathfrak{g}-\mathfrak{T}_{\mathfrak{g}}^{(\alpha)}=\left(\Omega, \mathfrak{g}-\mathcal{T}_{\mathfrak{g}}^{(\alpha)}\right)\right\rangle_{\alpha \in \Lambda}, \Lambda=\{\mathrm{K}, \mathrm{F}, \mathrm{H}, \mathrm{R}, \mathrm{N}\}$, denotes a sequence of $\mathfrak{g}-\mathcal{T}_{\mathfrak{g}}^{(\alpha)}$-spaces, obtained after endowing a $\mathcal{T}_{\mathfrak{g}}$-space $\mathfrak{T}_{\mathfrak{g}}=\left(\Omega, \mathcal{T}_{\mathfrak{g}}\right)$ with the sequence of $\mathfrak{g}-\mathrm{T}_{\mathfrak{g}, \alpha}$-axioms $\left\langle\mathfrak{g}-\mathrm{T}_{\mathfrak{g}, \alpha}\right\rangle_{\alpha \in \Lambda}$, then the following relations hold:

- I. $\mathfrak{T}_{\mathfrak{g}}^{(\mathrm{K})} \subseteq \mathfrak{T}_{\mathfrak{g}}^{(\mathrm{F})} \subseteq \mathfrak{T}_{\mathfrak{g}}^{(\mathrm{H})} \subseteq \mathfrak{T}_{\mathfrak{g}}^{(\mathrm{R})} \subseteq \mathfrak{T}_{\mathfrak{g}}^{(\mathrm{N})}$.

- II. $\mathfrak{g}-\mathrm{T}_{\mathfrak{g}, \mathrm{N}} \Rightarrow \mathfrak{g}-\mathrm{T}_{\mathfrak{g}, \mathrm{R}} \Rightarrow \mathfrak{g}-\mathrm{T}_{\mathfrak{g}, \mathrm{H}} \Rightarrow \mathfrak{g}-\mathrm{T}_{\mathfrak{g}, \mathrm{F}} \Rightarrow \mathfrak{g}-\mathrm{T}_{\mathfrak{g}, \mathrm{K}}$.

\section{DisCusSION}

4.1. Categorical Classifications. Having adopted a categorical approach in the classifications of the $\mathfrak{g}-T_{\mathfrak{g}, \alpha}$-axioms, $\alpha \in \Lambda=\{\mathrm{K}, \mathrm{F}, \mathrm{H}, \mathrm{R}, \mathrm{N}\}$, in the $\mathcal{T}_{\mathfrak{g}}$-space $\mathfrak{T}_{\mathfrak{g}}$, the aims here are, to establish the various relationships amongst the elements of the sequence $\left\langle\mathfrak{g}-\mathrm{T}_{\mathfrak{g}, \alpha}\right\rangle_{\alpha \in \Lambda}$ and, to illustrate them through diagrams.

We have seen that, both the $\mathfrak{g}-T_{\mathfrak{g}, N}, \mathfrak{g}-T_{\mathfrak{g}, R}$-axioms imply the $\mathfrak{g}-T_{\mathfrak{g}, K}, \mathfrak{g}-T_{\mathfrak{g}, F^{-}}$ axioms and, on the other hand, the $\mathfrak{g}-\mathrm{T}_{\mathfrak{g}, \mathrm{N}}$-axiom implies the $\mathfrak{g}-\mathrm{T}_{\mathfrak{g}, R}$-axiom and the

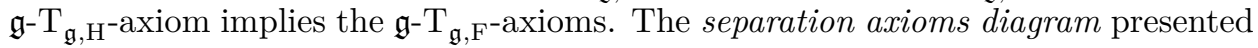
in FIGS 1 illustrates these implications.

We called the elements of the sequence $\left\langle\mathfrak{g}-\mathrm{T}_{\mathfrak{g}, \alpha}\right\rangle_{\alpha \in \Lambda} \mathfrak{g}-\mathrm{T}_{\mathfrak{g}, \alpha}$-axioms. To this end it does make sense to call those of $\left\langle\mathrm{T}_{\mathfrak{g}, \alpha}\right\rangle_{\alpha \in \Lambda} \mathrm{T}_{\mathfrak{g}, \alpha}$-axioms. Thus, in a $\mathcal{T}_{\mathfrak{g}}$-space $\mathfrak{T}_{\mathfrak{g}}=\left(\Omega, \mathcal{T}_{\mathfrak{g}}\right),\left\langle\mathrm{T}_{\mathfrak{g}, \alpha}\right\rangle_{\alpha \in \Lambda}$ stands for a sequence of separation axioms in the ordinary sense while $\left\langle\mathfrak{g}-\mathrm{T}_{\mathfrak{g}, \alpha}\right\rangle_{\alpha \in \Lambda}$ stands for its analogue but in the generalized sense, just as, in a $\mathcal{T}$-space $\mathfrak{T}=(\Omega, \mathcal{T}),\left\langle\mathrm{T}_{\alpha}\right\rangle_{\alpha \in \Lambda}$ stands for a sequence of separation axioms in the ordinary sense while $\left\langle\mathfrak{g}-\mathrm{T}_{\alpha}\right\rangle_{\alpha \in \Lambda}$ stands for its analogue but in the generalized 


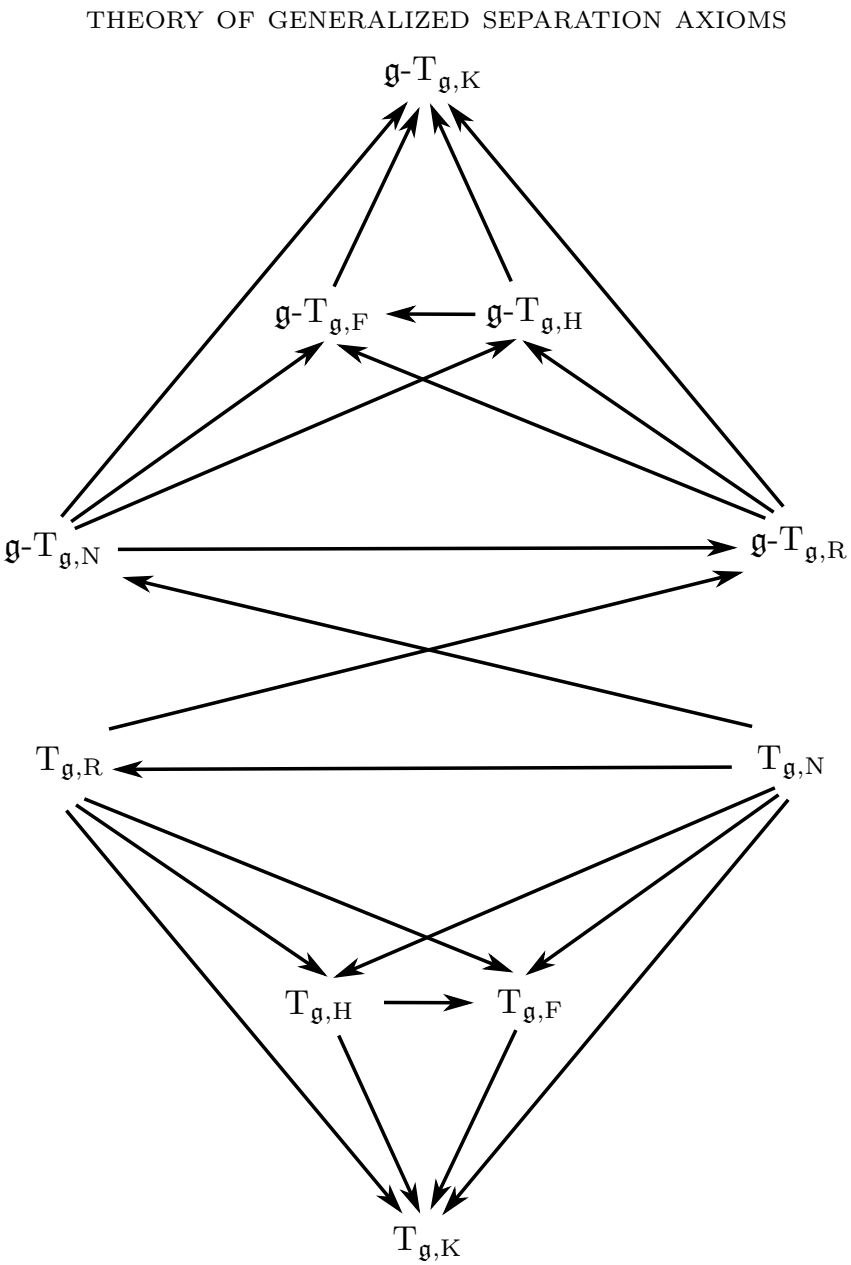

FiguRE 1. Relationships: Separation axioms diagram.

sense. Let $\mathrm{F}_{\mathrm{P}}[\sigma]=\mathfrak{g}-\mathrm{F}_{\mathrm{P}}[\sigma]$ and set

$$
\begin{aligned}
& \mathrm{F}_{\mathrm{O}}\left[\mathcal{S}_{\mathfrak{g}}\right] \stackrel{\text { def }}{=}\left\{\left(\mathcal{O}_{\mathfrak{g}, \xi}, \mathcal{O}_{\mathfrak{g}, \zeta}\right) \in \mathcal{T}_{\mathfrak{g}} \times \mathcal{T}_{\mathfrak{g}}: \bigcap_{\lambda=\xi, \zeta} \mathcal{O}_{\mathfrak{g}, \lambda} \subseteq \mathcal{S}_{\mathfrak{g}}\right\}, \\
& \mathrm{F}_{\mathrm{K}}\left[\mathcal{S}_{\mathfrak{g}}\right] \stackrel{\text { def }}{=}\left\{\left(\mathcal{K}_{\mathfrak{g}, \xi}, \mathcal{K}_{\mathfrak{g}, \zeta}\right) \in \neg \mathcal{T}_{\mathfrak{g}} \times \neg \mathcal{T}_{\mathfrak{g}}: \mathcal{S}_{\mathfrak{g}} \supseteq \bigcap_{\lambda=\xi, \zeta} \mathcal{K}_{\mathfrak{g}, \lambda}\right\},
\end{aligned}
$$

where $\sigma \geq 0$ and $\mathcal{S}_{\mathfrak{g}} \supseteq \emptyset$. Then, the notions of $\mathrm{T}_{\mathfrak{g}, \mathrm{K}}, \mathrm{T}_{\mathfrak{g}, \mathrm{F}}, \mathrm{T}_{\mathfrak{g}, \mathrm{H}}, \mathrm{T}_{\mathfrak{g}, \mathrm{R}}$, and $\mathrm{T}_{\mathfrak{g}, \mathrm{N}^{-}}$ axioms in a $\mathcal{T}_{\mathfrak{g}}$-space $\mathfrak{T}_{\mathfrak{g}}=\left(\Omega, \mathcal{T}_{\mathfrak{g}}\right)$ may well be defined as follows:

- I. $\mathrm{T}_{\mathfrak{g}, \mathrm{K}^{-}}$Axiom: For every $(\xi, \zeta) \in \mathrm{F}_{\mathrm{P}}[\sigma>0]$, there exists $\left(\mathcal{O}_{\mathfrak{g}, \xi}, \mathcal{O}_{\mathfrak{g}, \zeta}\right) \in$ $\mathrm{F}_{\mathrm{O}}\left[\mathcal{S}_{\mathfrak{g}} \supset \emptyset\right]$ such that:

$$
\left[\left(\xi \in \mathcal{O}_{\mathfrak{g}, \xi}\right) \wedge\left(\zeta \notin \mathcal{O}_{\mathfrak{g}, \xi}\right)\right] \vee\left[\left(\xi \notin \mathcal{O}_{\mathfrak{g}, \zeta}\right) \wedge\left(\zeta \in \mathcal{O}_{\mathfrak{g}, \zeta}\right)\right]
$$

- II. $\mathrm{T}_{\mathfrak{g}, \mathrm{F}}$-AxIOM: For every $(\xi, \zeta) \in \mathrm{F}_{\mathrm{P}}[\sigma>0]$, there exists $\left(\mathcal{O}_{\mathfrak{g}, \xi}, \mathcal{O}_{\mathfrak{g}, \zeta}\right) \in$ $\mathrm{F}_{\mathrm{O}}\left[\mathcal{S}_{\mathfrak{g}} \supset \emptyset\right]$ such that:

$$
\left[(\xi, \zeta) \in_{\lambda=\xi, \zeta} \mathcal{O}_{\mathfrak{g}, \lambda}\right] \wedge\left[(\xi, \zeta) \notin_{\lambda=\zeta, \xi} \mathcal{O}_{\mathfrak{g}, \lambda}\right] .
$$


- III. $\mathrm{T}_{\mathfrak{g}, \mathrm{H}^{-}}$AxiOM: For every $(\xi, \zeta) \in \mathrm{F}_{\mathrm{P}}[\sigma>0]$, there exists $\left(\mathcal{O}_{\mathfrak{g}, \xi}, \mathcal{O}_{\mathfrak{g}, \zeta}\right) \in$ $\mathrm{F}_{\mathrm{O}}[\emptyset]$ such that:

$$
\left[\xi \in \mathcal{O}_{\mathfrak{g}, \xi}\right] \wedge\left[\zeta \in \mathcal{O}_{\mathfrak{g}, \zeta}\right]
$$

- IV. $\mathrm{T}_{\mathfrak{g}, \mathrm{R}}$-Axiom: For every $(\xi, \zeta) \in \mathrm{F}_{\mathrm{P}}[\sigma>0]$ and $\left(\mathcal{K}_{\mathfrak{g}, \xi}, \mathcal{K}_{\mathfrak{g}, \zeta}\right) \in \mathrm{F}_{\mathrm{K}}[\emptyset]$ such that $(\zeta, \xi) \notin\left(\mathcal{K}_{\mathfrak{g}, \xi}, \mathcal{K}_{\mathfrak{g}, \zeta}\right)$, there exists $\left(\mathcal{O}_{\mathfrak{g}, \xi}, \mathcal{O}_{\mathfrak{g}, \zeta}\right) \in \mathrm{F}_{\mathrm{O}}[\emptyset]$ such that:

$$
\left[\left(\mathcal{K}_{\mathfrak{g}, \xi} \subset \mathcal{O}_{\mathfrak{g}, \xi}\right) \wedge\left(\zeta \in \mathcal{O}_{\mathfrak{g}, \zeta}\right)\right] \vee\left[\left(\mathcal{K}_{\mathfrak{g}, \zeta} \subset \mathcal{O}_{\mathfrak{g}, \zeta}\right) \wedge\left(\xi \in \mathcal{O}_{\mathfrak{g}, \xi}\right)\right]
$$

- v. $\mathrm{T}_{\mathfrak{g}, \mathrm{N}^{-}}$-Axiom: For every $\left(\mathcal{K}_{\mathfrak{g}, \xi}, \mathcal{K}_{\mathfrak{g}, \zeta}\right) \in \mathrm{F}_{\mathrm{K}}[\emptyset]$, there exists $\left(\mathcal{O}_{\mathfrak{g}, \xi}, \mathcal{O}_{\mathfrak{g}, \zeta}\right) \in$ $\mathrm{F}_{\mathrm{O}}[\emptyset]$ such that:

$$
\left[\mathcal{O}_{\mathfrak{g}, \xi} \supset \mathcal{K}_{\mathfrak{g}, \xi}\right] \wedge\left[\mathcal{O}_{\mathfrak{g}, \zeta} \supset \mathcal{K}_{\mathfrak{g}, \zeta}\right]
$$

By virtue of the relations $\mathcal{O}_{\mathfrak{g}, \lambda} \subseteq \mathrm{op}_{\mathfrak{g}}\left(\mathcal{O}_{\mathfrak{g}, \lambda}\right)$ and $\mathcal{K}_{\mathfrak{g}, \lambda} \supseteq \neg \mathrm{op}_{\mathfrak{g}}\left(\mathcal{K}_{\mathfrak{g}, \lambda}\right)$ for every $\left(\mathcal{O}_{\mathfrak{g}, \lambda}, \mathcal{K}_{\mathfrak{g}, \lambda}, \lambda\right) \in \mathcal{T}_{\mathfrak{g}} \times \neg \mathcal{T}_{\mathfrak{g}} \times\{\xi, \zeta\}$, these implications follow: $\mathfrak{g}-\mathrm{T}_{\mathfrak{g}, \mathrm{K}} \longleftarrow \mathrm{T}_{\mathfrak{g}, \mathrm{K}}$, $\mathfrak{g}-\mathrm{T}_{\mathfrak{g}, \mathrm{F}} \longleftarrow \mathrm{T}_{\mathfrak{g}, \mathrm{F}}, \mathfrak{g}-\mathrm{T}_{\mathfrak{g}, \mathrm{H}} \longleftarrow \mathrm{T}_{\mathfrak{g}, \mathrm{H}}, \mathfrak{g}-\mathrm{T}_{\mathfrak{g}, \mathrm{R}} \longleftarrow \mathrm{T}_{\mathfrak{g}, \mathrm{R}}$, and $\mathfrak{g}-\mathrm{T}_{\mathfrak{g}, \mathrm{N}} \longleftarrow \mathrm{T}_{\mathfrak{g}, \mathrm{N}}$. When the statements preceding the above definitions are taken into account, another separation axioms diagram is obtained. In Fig. 2, we have illustrated the various relationships amongst the elements of $\left\langle\mathfrak{g}-\mathrm{T}_{\mathfrak{g}, \alpha}\right\rangle_{\alpha \in \Lambda}$ and $\left\langle\mathrm{T}_{\mathfrak{g}, \alpha}\right\rangle_{\alpha \in \Lambda}$. It is interesting to present a third separation axioms diagram illustrating both the implications and the categorical classifications of the elements of $\left\langle\mathfrak{g}-\nu-\mathrm{T}_{\mathfrak{g}, \alpha}\right\rangle_{\alpha \in \Lambda}$, where $\nu \in I_{3}^{0}$.

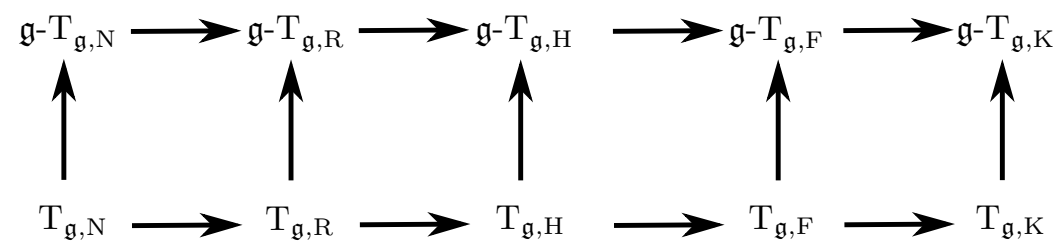

FiguRE 2. Relationships: Separation axioms diagram.

For every fixed $\nu \in I_{3}^{0}$, it is immediate that the implications $\mathfrak{g}-\nu-\mathrm{T}_{\mathfrak{g}, \mathrm{K}} \longleftarrow$ $\mathfrak{g}-\nu-T_{\mathfrak{g}, \mathrm{F}}, \mathfrak{g}-\nu-\mathrm{T}_{\mathfrak{g}, \mathrm{F}} \longleftarrow \mathfrak{g}-\nu-\mathrm{T}_{\mathfrak{g}, \mathrm{H}}, \mathfrak{g}-\nu-\mathrm{T}_{\mathfrak{g}, \mathrm{H}} \longleftarrow \mathfrak{g}-\nu-\mathrm{T}_{\mathfrak{g}, \mathrm{R}}$, and $\mathfrak{g}-\nu-\mathrm{T}_{\mathfrak{g}, \mathrm{R}} \longleftarrow \mathfrak{g}-\nu-T_{\mathfrak{g}, \mathrm{N}}$ hold. On the other hand, we saw in the first part of our works, on the theory of $\mathfrak{g}-\mathfrak{T}_{\mathfrak{g}}$-sets, that

$$
\begin{aligned}
\operatorname{op}_{\mathfrak{g}, 0}\left(\mathcal{S}_{\mathfrak{g}}\right) \subseteq \mathrm{op}_{\mathfrak{g}, 1}\left(\mathcal{S}_{\mathfrak{g}}\right) \subseteq \mathrm{op}_{\mathfrak{g}, 3}\left(\mathcal{S}_{\mathfrak{g}}\right) \supseteq \mathrm{op}_{\mathfrak{g}, 2}\left(\mathcal{S}_{\mathfrak{g}}\right) \forall \mathcal{S}_{\mathfrak{g}} \subset \mathfrak{T}_{\mathfrak{g}}, \\
\neg \mathrm{op}_{\mathfrak{g}, 0}\left(\mathcal{S}_{\mathfrak{g}}\right) \supseteq \neg \mathrm{op}_{\mathfrak{g}, 1}\left(\mathcal{S}_{\mathfrak{g}}\right) \supseteq \neg \mathrm{op}_{\mathfrak{g}, 3}\left(\mathcal{S}_{\mathfrak{g}} \subseteq \neg \mathrm{op}_{\mathfrak{g}, 2}\left(\mathcal{S}_{\mathfrak{g}}\right),\right.
\end{aligned}
$$

as a consequence of the definitions of the $\mathfrak{g}$-operators $\mathrm{op}_{\mathfrak{g}, \nu}, \neg \mathrm{op}_{\mathfrak{g}, \nu}: \mathcal{P}(\Omega) \longrightarrow$ $\mathcal{P}(\Omega)$. Hence, it results that, for every $\alpha \in \Lambda, \mathfrak{g}-0-\mathrm{T}_{\mathfrak{g}, \alpha} \longrightarrow \mathfrak{g}-1-\mathrm{T}_{\mathfrak{g}, \alpha} \longrightarrow \mathfrak{g}-3-\mathrm{T}_{\mathfrak{g}, \alpha}$ and $\mathfrak{g}-3-\mathrm{T}_{\mathfrak{g}, \alpha} \longleftarrow \mathfrak{g}-2-\mathrm{T}_{\mathfrak{g}, \alpha}$. When these properties are taken into consideration, the resulting separation axioms diagram so obtained is that presented in FIG. 3. It is reasonable to call them $\mathfrak{g}-\mathrm{T}_{\mathfrak{g}, \alpha}$-axioms of type $\alpha$ and of category $\nu$, where $(\alpha, \nu) \in \Lambda \times I_{3}^{0}$.

In order to exemplify the concept of $\mathfrak{g}$ - $\mathrm{T}_{\mathfrak{g}, \alpha}$-axiom of type $\alpha$ and of category $\nu$, where $(\alpha, \nu) \in \Lambda \times I_{3}^{0}$, a nice application is presented in the following section. 


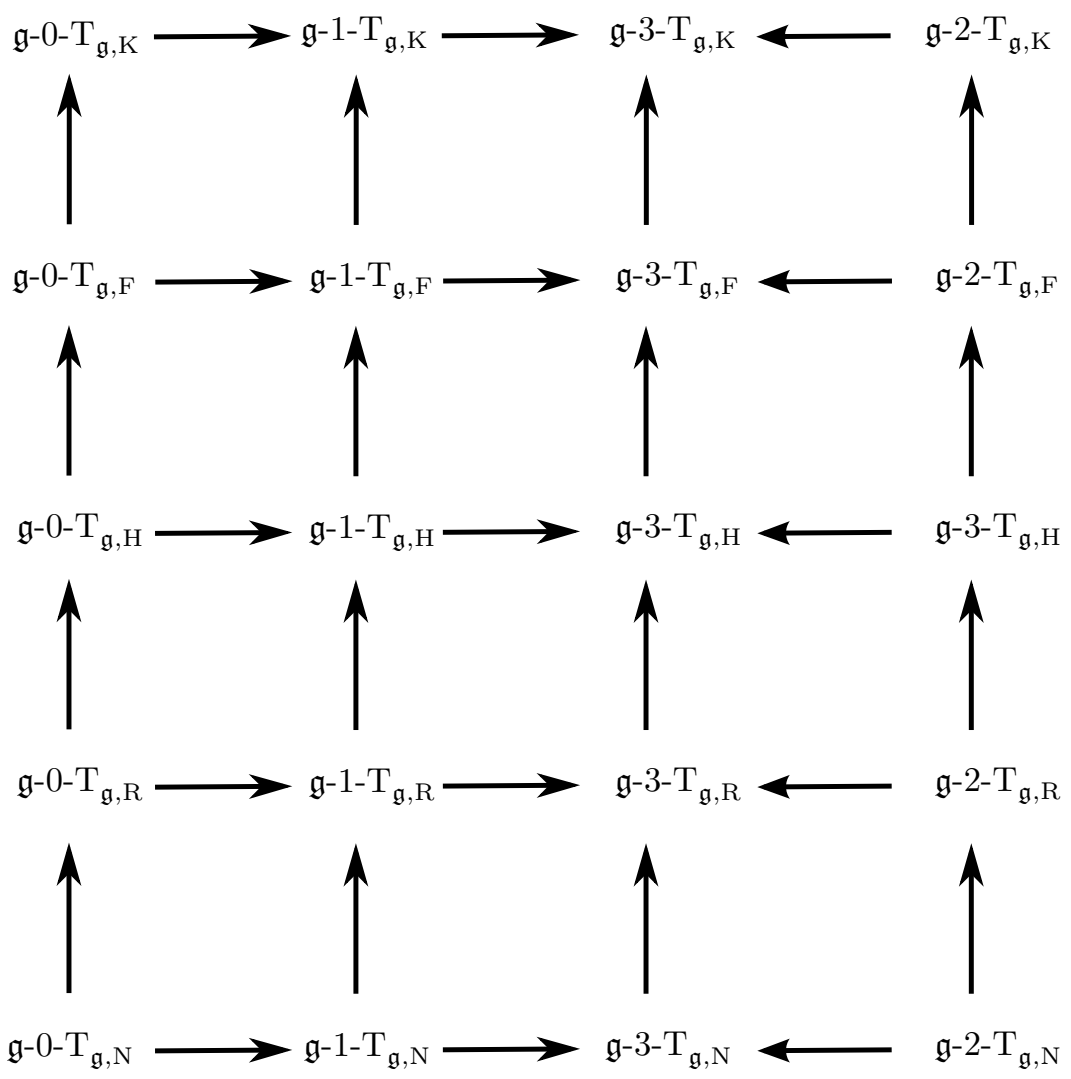

Figure 3. Relationships: Separation axiom diagram.

4.2. A Nice Application. Focusing on the fundamental notions of the sequence $\left\langle\mathfrak{g}-\mathrm{T}_{\mathfrak{g}, \alpha}\right\rangle_{\alpha \in \Lambda}$ of $\mathfrak{g}$ - $\mathrm{T}_{\mathfrak{g}, \alpha}$-axioms, $\Lambda=\{\mathrm{K}, \mathrm{F}, \mathrm{H}, \mathrm{R}, \mathrm{N}\}$, in a $\mathcal{T}_{\mathfrak{g}}$-space, founded upon the class of $\mathfrak{g}-\mathcal{T}_{\mathfrak{g}}$-open sets, we shall now present a nice application. Let $\Omega=\left\{\xi_{\nu}\right.$ : $\left.\nu \in I_{3}^{*}\right\}$ denotes the underlying set and consider the $\mathcal{T}_{\mathfrak{g}}$-space $\mathfrak{T}_{\mathfrak{g}}=\left(\Omega, \mathcal{T}_{\mathfrak{g}}\right)$, where

$$
\begin{aligned}
\mathcal{T}_{\mathfrak{g}}(\Omega) & =\left\{\emptyset,\left\{\xi_{1}\right\},\left\{\xi_{2}\right\},\left\{\xi_{3}\right\},\left\{\xi_{1}, \xi_{2}\right\},\left\{\xi_{1}, \xi_{3}\right\},\left\{\xi_{2}, \xi_{3}\right\}, \Omega\right\} \\
& =\left\{\mathcal{O}_{\mathfrak{g}, 1}, \mathcal{O}_{\mathfrak{g}, 2}, \mathcal{O}_{\mathfrak{g}, 3}, \mathcal{O}_{\mathfrak{g}, 4}, \mathcal{O}_{\mathfrak{g}, 5}, \mathcal{O}_{\mathfrak{g}, 6}, \mathcal{O}_{\mathfrak{g}, 7}, \mathcal{O}_{\mathfrak{g}, 8}\right\} \\
\neg \mathcal{T}_{\mathfrak{g}}(\Omega) & =\left\{\Omega,\left\{\xi_{2}, \xi_{3}\right\},\left\{\xi_{1}, \xi_{3}\right\},\left\{\xi_{1}, \xi_{2}\right\},\left\{\xi_{3}\right\},\left\{\xi_{2}\right\},\left\{\xi_{1}\right\}, \emptyset\right\} \\
& =\left\{\mathcal{K}_{\mathfrak{g}, 1}, \mathcal{K}_{\mathfrak{g}, 2}, \mathcal{K}_{\mathfrak{g}, 3}, \mathcal{K}_{\mathfrak{g}, 4}, \mathcal{K}_{\mathfrak{g}, 5}, \mathcal{K}_{\mathfrak{g}, 6}, \mathcal{K}_{\mathfrak{g}, 7}, \mathcal{K}_{\mathfrak{g}, 8}\right\}
\end{aligned}
$$

respectively, stand for the classes of $\mathcal{T}_{\mathfrak{g}}$-open and $\mathcal{T}_{\mathfrak{g}}$-closed sets. In both settings, the $\mathcal{T}_{\mathfrak{g}}$-open, closed sets occupying the $\nu^{\text {th }}$ position corresponds to $\mathcal{O}_{\mathfrak{g}, \nu}, \mathcal{K}_{\mathfrak{g}, \nu}, \nu \in I_{8}^{*}$, respectively, as is easily understood. Since conditions $\mathcal{T}_{\mathfrak{g}}(\emptyset)=\emptyset, \mathcal{T}_{\mathfrak{g}}\left(\mathcal{O}_{\mathfrak{g}, \nu}\right) \subseteq \mathcal{O}_{\mathfrak{g}, \nu}$ for every $\nu \in I_{8}^{*}$, and $\mathcal{T}_{\mathfrak{g}}\left(\bigcup_{\nu \in I_{8}^{*}} \mathcal{O}_{\mathfrak{g}, \nu}\right)=\bigcup_{\nu \in I_{8}^{*}} \mathcal{T}_{\mathfrak{g}}\left(\mathcal{O}_{\mathfrak{g}, \nu}\right)$ are satisfied, it is clear that the one-valued map $\mathcal{T}_{\mathfrak{g}}: \mathcal{P}(\Omega) \longrightarrow \mathcal{P}\left(\left\{\xi_{\nu}: \nu \in I_{8}^{*}\right\}\right)$ is a $\mathfrak{g}$-topology. After 
computing the elements of the set $\left\{\operatorname{op}_{\mathfrak{g}, \nu}\left(\mathcal{O}_{\mathfrak{g}, \xi_{\alpha}}\right):(\alpha, \nu) \in I_{8}^{*} \times I_{3}^{0}\right\}$, called $\mathfrak{g}-\mathcal{T}_{\mathfrak{g}^{-}}$ open sets, we obtain:

$$
\mathrm{op}_{\mathfrak{g}, \nu}\left(\mathcal{O}_{\mathfrak{g}, \xi_{\alpha}}\right) \in \begin{cases}\left\{\mathcal{O}_{\mathfrak{g}, 2}, \mathcal{O}_{\mathfrak{g}, 5}, \mathcal{O}_{\mathfrak{g}, 6}, \mathcal{O}_{\mathfrak{g}, 8}\right\} & \forall(\alpha, \nu) \in\{1\} \times\{0,2\}, \\ \left\{\mathcal{O}_{\mathfrak{g}, 3}, \mathcal{O}_{\mathfrak{g}, 5}, \mathcal{O}_{\mathfrak{g}, 7}, \mathcal{O}_{\mathfrak{g}, 8}\right\} & \forall(\alpha, \nu) \in\{2\} \times\{0,2\}, \\ \left\{\mathcal{O}_{\mathfrak{g}, 4}, \mathcal{O}_{\mathfrak{g}, 6}, \mathcal{O}_{\mathfrak{g}, 7}, \mathcal{O}_{\mathfrak{g}, 8}\right\} & \forall(\alpha, \nu) \in\{3\} \times\{0,2\}, \\ \left\{\mathcal{K}_{\mathfrak{g}, 1}, \mathcal{K}_{\mathfrak{g}, 3}, \mathcal{K}_{\mathfrak{g}, 4}, \mathcal{K}_{\mathfrak{g}, 7}\right\} & \forall(\alpha, \nu) \in\{1\} \times\{1,3\}, \\ \left\{\mathcal{K}_{\mathfrak{g}, 1}, \mathcal{K}_{\mathfrak{g}, 2}, \mathcal{K}_{\mathfrak{g}, 4}, \mathcal{K}_{\mathfrak{g}, 6}\right\} & \forall(\alpha, \nu) \in\{2\} \times\{1,3\}, \\ \left\{\mathcal{K}_{\mathfrak{g}, 1}, \mathcal{K}_{\mathfrak{g}, 2}, \mathcal{K}_{\mathfrak{g}, 3}, \mathcal{K}_{\mathfrak{g}, 5}\right\} & \forall(\alpha, \nu) \in\{3\} \times\{1,3\} .\end{cases}
$$

Similarly, the elements of $\left\{\neg \mathrm{op}_{\mathfrak{g}, \nu}\left(\mathcal{K}_{\mathfrak{g}, \xi_{\alpha}}\right):(\alpha, \nu) \in I_{8}^{*} \times I_{3}^{0}\right\}$, called $\mathfrak{g}$ - $\mathcal{T}_{\mathfrak{g}}$-closed sets, are:

$$
\neg \operatorname{op}_{\mathfrak{g}, \nu}\left(\mathcal{K}_{\mathfrak{g}, \xi_{\alpha}}\right) \in \begin{cases}\left\{\mathcal{K}_{\mathfrak{g}, 1}, \mathcal{K}_{\mathfrak{g}, 3}, \mathcal{K}_{\mathfrak{g}, 4}, \mathcal{K}_{\mathfrak{g}, 7}\right\} & \forall(\alpha, \nu) \in\{1\} \times\{0,2\}, \\ \left\{\mathcal{K}_{\mathfrak{g}, 1}, \mathcal{K}_{\mathfrak{g}, 2}, \mathcal{K}_{\mathfrak{g}, 4}, \mathcal{K}_{\mathfrak{g}, 6}\right\} & \forall(\alpha, \nu) \in\{2\} \times\{0,2\}, \\ \left\{\mathcal{K}_{\mathfrak{g}, 1}, \mathcal{K}_{\mathfrak{g}, 2}, \mathcal{K}_{\mathfrak{g}, 3}, \mathcal{K}_{\mathfrak{g}, 5}\right\} & \forall(\alpha, \nu) \in\{3\} \times\{0,2\}, \\ \left\{\mathcal{O}_{\mathfrak{g}, 2}, \mathcal{O}_{\mathfrak{g}, 5}, \mathcal{O}_{\mathfrak{g}, 6}, \mathcal{O}_{\mathfrak{g}, 8}\right\} & \forall(\alpha, \nu) \in\{1\} \times\{1,3\}, \\ \left\{\mathcal{O}_{\mathfrak{g}, 3}, \mathcal{O}_{\mathfrak{g}, 5}, \mathcal{O}_{\mathfrak{g}, 7}, \mathcal{O}_{\mathfrak{g}, 8}\right\} & \forall(\alpha, \nu) \in\{2\} \times\{1,3\}, \\ \left\{\mathcal{O}_{\mathfrak{g}, 4}, \mathcal{O}_{\mathfrak{g}, 6}, \mathcal{O}_{\mathfrak{g}, 7}, \mathcal{O}_{\mathfrak{g}, 8}\right\} & \forall(\alpha, \nu) \in\{3\} \times\{1,3\} .\end{cases}
$$

First, for every $\nu \in I_{3}^{0}$, set $\mathcal{I}_{\mathfrak{g},\left(\xi_{\alpha}, \xi_{\beta}\right)}^{\mathrm{op}}=\bigcap_{\lambda=\alpha, \beta} \mathrm{op}_{\mathfrak{g}, \nu}\left(\mathcal{O}_{\mathfrak{g}, \xi_{\lambda}}\right)$ and $\mathcal{I}_{\mathfrak{g},\left(\xi_{\alpha}, \xi_{\beta}\right)}^{\mathrm{cl}}=$ $\bigcap_{\lambda=\alpha, \beta} \neg \mathrm{op}_{\mathfrak{g}, \nu}\left(\mathcal{K}_{\mathfrak{g}, \xi_{\lambda}}\right)$. Next, for all $(\alpha, \beta, \nu) \in I_{3}^{*} \times I_{3}^{*} \times I_{3}^{0}$, calculate $\mathcal{I}_{\mathfrak{g},\left(\xi_{\alpha}, \xi_{\beta}\right)}^{\mathrm{op}}$, $\mathcal{I}_{\mathfrak{g},\left(\xi_{\alpha}, \xi_{\beta}\right)}^{\text {cl }}$. Finally, for every $(r, s) \in I_{8}^{*} \times I_{8}^{*}$, set $\mathcal{O}_{\mathfrak{g},(r, s)}=\left(\mathcal{O}_{\mathfrak{g}, r}, \mathcal{O}_{\mathfrak{g}, s}\right)$ and $\mathcal{K}_{\mathfrak{g},(r, s)}=\left(\mathcal{K}_{\mathfrak{g}, r}, \mathcal{K}_{\mathfrak{g}, s}\right)$. These procedures yield:

$$
\begin{aligned}
& \mathfrak{g}-\mathrm{F}_{\mathrm{P}}[\sigma>0]=\bigcup_{\alpha \in I_{3}^{*}}\left\{\left(\xi_{\alpha}, \xi_{\beta}\right): \beta \in I_{3}^{*} \backslash\{\alpha\}\right\}, \\
& \mathfrak{g}-\nu-\mathrm{F}_{\mathrm{O}}[\emptyset]=\left\{\mathcal{O}_{\mathfrak{g},(3,2)}, \mathcal{O}_{\mathfrak{g},(3,6)}, \mathcal{O}_{\mathfrak{g},(4,2)}, \mathcal{O}_{\mathfrak{g},(4,3)},\right. \\
& \left.\mathcal{O}_{\mathfrak{g},(4,5)}, \mathcal{O}_{\mathfrak{g},(6,3)}, \mathcal{O}_{\mathfrak{g},(7,2)}\right\}, \\
& \mathfrak{g}-\nu-\mathrm{F}_{\mathrm{K}}[\emptyset]=\left\{\mathcal{K}_{\mathfrak{g},(2,7)}, \mathcal{K}_{\mathfrak{g},(3,6)}, \mathcal{K}_{\mathfrak{g},(5,4)}, \mathcal{K}_{\mathfrak{g},(5,6)},\right. \\
& \left.\mathcal{K}_{\mathfrak{g},(5,7)}, \mathcal{K}_{\mathfrak{g},(6,3)}, \mathcal{K}_{\mathfrak{g},(6,7)}\right\}, \\
& \mathfrak{g}-\nu-\mathrm{F}_{\mathrm{O}}\left[\mathcal{S}_{\mathfrak{g}} \supset \emptyset\right]=\left\{\mathcal{O}_{\mathfrak{g},(r, s)}:(r, s) \in I_{8}^{*} \times I_{8}^{*}\right\} \supset \mathfrak{g}-\nu-\mathrm{F}_{\mathrm{O}}[\emptyset] \quad \forall \nu \in I_{3}^{0}, \\
& \mathfrak{g}-\nu-\mathrm{F}_{\mathrm{K}}\left[\mathcal{S}_{\mathfrak{g}} \supset \emptyset\right]=\left\{\mathcal{K}_{\mathfrak{g},(r, s)}:(r, s) \in I_{8}^{*} \times I_{8}^{*}\right\} \supset \mathfrak{g}-\nu-\mathrm{F}_{\mathrm{K}}[\emptyset] .
\end{aligned}
$$

We are now in a position to discuss the $\mathfrak{g}$ - $\mathrm{T}_{\mathfrak{g}, \alpha}$-axioms, $\Lambda=\{\mathrm{K}, \mathrm{F}, \mathrm{H}, \mathrm{R}, \mathrm{N}\}$.

Let $\mathcal{O}_{\mathfrak{g},(p, q)} \supset \mathcal{K}_{\mathfrak{g},(r, s)}$ stand for the relations $\mathcal{O}_{\mathfrak{g}, p} \supset \mathcal{K}_{\mathfrak{g}, r}$ and $\mathcal{O}_{\mathfrak{g}, q} \supset \mathcal{K}_{\mathfrak{g}, s}$, where $\mathcal{O}_{\mathfrak{g},(p, q)} \in \mathfrak{g}-\nu-\mathrm{F}_{\mathrm{O}}[\emptyset]$ and $\mathcal{K}_{\mathfrak{g},(r, s)} \in \mathfrak{g}-\nu-\mathrm{F}_{\mathrm{K}}[\emptyset]$. Further, for every $\nu \in$ $I_{3}^{0}$, let $\mathrm{op}_{\mathfrak{g}, \nu}\left(\mathcal{O}_{\mathfrak{g},(p, q)}\right) \supset \neg \mathrm{op}_{\mathfrak{g}, \nu}\left(\mathcal{K}_{\mathfrak{g},(r, s)}\right)$ stand for $\operatorname{op}_{\mathfrak{g}, \nu}\left(\mathcal{O}_{\mathfrak{g}, p}\right) \supset \neg \mathrm{op}_{\mathfrak{g}, \nu}\left(\mathcal{K}_{\mathfrak{g}, r}\right)$, $\mathrm{op}_{\mathfrak{g}, \nu}\left(\mathcal{O}_{\mathfrak{g}, q}\right) \supset \neg \mathrm{op}_{\mathfrak{g}, \nu}\left(\mathcal{K}_{\mathfrak{g}, s}\right)$. Then, the following relations are easily checked: 
$\mathcal{O}_{\mathfrak{g},(7,2)} \supset \mathcal{K}_{\mathfrak{g},(2,7)} ; \mathcal{O}_{\mathfrak{g},(6,3)} \supset \mathcal{K}_{\mathfrak{g},(3,6)} ; \mathcal{O}_{\mathfrak{g},(4,5)} \supset \mathcal{K}_{\mathfrak{g},(5,4)} ; \mathcal{O}_{\mathfrak{g},(4,3)} \supset \mathcal{K}_{\mathfrak{g},(5,6)} ;$ $\mathcal{O}_{\mathfrak{g},(4,2)} \supset \mathcal{K}_{\mathfrak{g},(5,7)} ; \mathcal{O}_{\mathfrak{g},(3,6)} \supset \mathcal{K}_{\mathfrak{g},(6,3)}$ and $\mathcal{O}_{\mathfrak{g},(3,2)} \supset \mathcal{K}_{\mathfrak{g},(6,7)}$. But, for every $\nu \in I_{3}^{0}$, the relations $\mathcal{O}_{\mathfrak{g},(p, q)} \subseteq \mathrm{op}_{\mathfrak{g}, \nu}\left(\mathcal{O}_{\mathfrak{g},(p, q)}\right)$ and $\mathcal{K}_{\mathfrak{g},(r, s)} \supseteq \neg \mathrm{op}_{\mathfrak{g}, \nu}\left(\mathcal{K}_{\mathfrak{g},(r, s)}\right)$ hold for all $(p, q)=(3,2),(3,6),(4,2),(4,3),(4,5),(6,3),(7,2)$ and all $(r, s)=(6,7)$, $(6,3),(5,7),(5,6),(5,4),(3,6),(2,7)$. Combining these last two relations with $\mathcal{O}_{\mathfrak{g},(p, q)} \supset \mathcal{K}_{\mathfrak{g},(r, s)}$, it follows that $\operatorname{op}_{\mathfrak{g}, \nu}\left(\mathcal{O}_{\mathfrak{g},(p, q)}\right) \supset \neg \mathrm{op}_{\mathfrak{g}, \nu}\left(\mathcal{K}_{\mathfrak{g},(r, s)}\right)$. Hence, for every $\mathcal{K}_{\mathfrak{g},(r, s)} \in \mathfrak{g}-\nu-\mathrm{F}_{\mathrm{K}}[\emptyset]$, there exists $\mathcal{O}_{\mathfrak{g},(p, q)} \in \mathfrak{g}-\nu-\mathrm{F}_{\mathrm{O}}[\emptyset]$ such that:

$$
\left[\operatorname{op}_{\mathfrak{g}, \nu}\left(\mathcal{O}_{\mathfrak{g}, p}\right) \supset \neg \mathrm{op}_{\mathfrak{g}, \nu}\left(\mathcal{K}_{\mathfrak{g}, r}\right)\right] \wedge\left[\mathrm{op}_{\mathfrak{g}, \nu}\left(\mathcal{O}_{\mathfrak{g}, q}\right) \supset \neg \mathrm{op}_{\mathfrak{g}, \nu}\left(\mathcal{K}_{\mathfrak{g}, s}\right)\right] .
$$

This shows that $\mathfrak{T}_{\mathfrak{g}}$ is a $\mathfrak{g}-\mathcal{T}_{\mathfrak{g}}^{(\mathrm{N})}$-space $\mathfrak{g}-\mathfrak{T}_{\mathfrak{g}}^{(\mathrm{N})}=\left(\Omega, \mathfrak{g}-\mathcal{T}_{\mathfrak{g}}^{(\mathrm{N})}\right)$.

Let $\left(\xi_{i}, \xi_{j}\right) \in \mathcal{K}_{\mathfrak{g},(r, s)}$ mean $\xi_{i} \in \mathcal{K}_{\mathfrak{g}, r}$ and $\xi_{j} \in \mathcal{K}_{\mathfrak{g}, s}$, where $\mathcal{K}_{\mathfrak{g},(r, s)} \in \mathfrak{g}-\nu-\mathrm{F}_{\mathrm{K}}[\emptyset]$. Then, the following results are easily checked: $\left(\xi_{2}, \xi_{1}\right) \in \mathcal{K}_{\mathfrak{g},(2,7)}, \mathcal{K}_{\mathfrak{g},(6,3)}, \mathcal{K}_{\mathfrak{g},(6,7)}$ and $\left(\xi_{1}, \xi_{2}\right) \notin \mathcal{K}_{\mathfrak{g},(2,7)}, \mathcal{K}_{\mathfrak{g},(6,3)}, \mathcal{K}_{\mathfrak{g},(6,7)} ;\left(\xi_{3}, \xi_{1}\right) \in \mathcal{K}_{\mathfrak{g},(2,7)}, \mathcal{K}_{\mathfrak{g},(5,4)}, \mathcal{K}_{\mathfrak{g},(5,7)}$ and $\left(\xi_{1}, \xi_{3}\right) \notin \mathcal{K}_{\mathfrak{g},(2,7)}, \mathcal{K}_{\mathfrak{g},(5,4)}, \mathcal{K}_{\mathfrak{g},(5,7)} ;\left(\xi_{3}, \xi_{2}\right) \in \mathcal{K}_{\mathfrak{g},(3,6)}, \mathcal{K}_{\mathfrak{g},(5,4)}, \mathcal{K}_{\mathfrak{g},(5,6)}$ and $\left(\xi_{2}, \xi_{3}\right) \notin$ $\mathcal{K}_{\mathfrak{g},(3,6)}, \mathcal{K}_{\mathfrak{g},(5,4)}, \mathcal{K}_{\mathfrak{g},(5,6)}$. But, $\mathcal{O}_{\mathfrak{g},(7,2)} \supset \mathcal{K}_{\mathfrak{g},(2,7)} ; \mathcal{O}_{\mathfrak{g},(6,3)} \supset \mathcal{K}_{\mathfrak{g},(3,6)} ; \mathcal{O}_{\mathfrak{g},(4,5)} \supset$ $\mathcal{K}_{\mathfrak{g},(5,4)} ; \mathcal{O}_{\mathfrak{g},(4,3)} \supset \mathcal{K}_{\mathfrak{g},(5,6)} ; \mathcal{O}_{\mathfrak{g},(4,2)} \supset \mathcal{K}_{\mathfrak{g},(5,7)} ; \mathcal{O}_{\mathfrak{g},(3,6)} \supset \mathcal{K}_{\mathfrak{g},(6,3)}$ and $\mathcal{O}_{\mathfrak{g},(3,2)} \supset$ $\mathcal{K}_{\mathfrak{g},(6,7)}$. Furthermore, for every $\nu \in I_{3}^{0}, \mathcal{O}_{\mathfrak{g},(p, q)} \subseteq \mathrm{op}_{\mathfrak{g}, \nu}\left(\mathcal{O}_{\mathfrak{g},(p, q)}\right)$ for all $(p, q)=$ $(3,2),(3,6),(4,2),(4,3),(4,5),(6,3),(7,2)$ and $\mathcal{K}_{\mathfrak{g},(r, s)} \supseteq \neg \mathrm{op}_{\mathfrak{g}, \nu}\left(\mathcal{K}_{\mathfrak{g},(r, s)}\right)$ for all $(r, s)=(2,7),(3,6),(5,4),(5,6),(5,7),(6,3),(6,7)$. Thus, for every $\left(\xi_{i}, \xi_{j}\right) \in$ $\mathfrak{g}-\mathrm{F}_{\mathrm{P}}[\sigma>0]$ and $\mathcal{K}_{\mathfrak{g},(r, s)} \in \mathfrak{g}-\nu-\mathrm{F}_{\mathrm{K}}[\emptyset]$ such that $\left(\xi_{j}, \xi_{i}\right) \notin \mathcal{K}_{\mathfrak{g},(r, s)}$, there exists $\mathcal{O}_{\mathfrak{g},(p, q)} \in \mathfrak{g}-\nu-\mathrm{F}_{\mathrm{O}}[\emptyset]$ such that:

$$
\begin{aligned}
{\left[\left(\neg \mathrm{op}_{\mathfrak{g}, \nu}\left(\mathcal{K}_{\mathfrak{g}, r}\right)\right.\right.} & \left.\subset \mathrm{op}_{\mathfrak{g}, \nu}\left(\mathcal{O}_{\mathfrak{g}, p}\right)\right) \\
\left.\subset \mathrm{op}_{\mathfrak{g}, \nu}\left(\mathcal{O}_{\mathfrak{g}, q}\right)\right) & \left.\wedge\left(\zeta \in\left(\xi \in \mathrm{op}_{\mathfrak{g}, \nu}\left(\mathcal{O}_{\mathfrak{g}, q}\right)\right)\right] \vee\left[\left(\neg \mathcal{O}_{\mathfrak{g}, p}\right)\right)\right] .
\end{aligned}
$$

This shows that $\mathfrak{T}_{\mathfrak{g}}$ is a $\mathfrak{g}-\mathcal{T}_{\mathfrak{g}}^{(\mathrm{R})}$-space $\mathfrak{g}-\mathfrak{T}_{\mathfrak{g}}^{(\mathrm{R})}=\left(\Omega, \mathfrak{g}-\mathcal{T}_{\mathfrak{g}}^{(\mathrm{R})}\right)$.

Let $\left(\xi_{i}, \xi_{j}\right) \in \mathcal{O}_{\mathfrak{g},(p, q)}$ mean $\xi_{i} \in \mathcal{O}_{\mathfrak{g}, p}$ and $\xi_{j} \in \mathcal{O}_{\mathfrak{g}, q}$, where $\mathcal{O}_{\mathfrak{g},(p, q)} \in \mathfrak{g}-\nu$ - $\mathrm{F}_{\mathrm{O}}[\emptyset]$. Then, the following relations are easily verified: $\left(\xi_{2}, \xi_{1}\right) \in \mathcal{O}_{\mathfrak{g},(3,2)}, \mathcal{O}_{\mathfrak{g},(3,6)}, \mathcal{O}_{\mathfrak{g},(7,2)}$ and $\left(\xi_{1}, \xi_{2}\right) \notin \mathcal{O}_{\mathfrak{g},(3,2)}, \mathcal{O}_{\mathfrak{g},(3,6)}, \mathcal{O}_{\mathfrak{g},(7,2)} ;\left(\xi_{3}, \xi_{1}\right) \in \mathcal{O}_{\mathfrak{g},(4,2)}, \mathcal{O}_{\mathfrak{g},(4,5)}, \mathcal{O}_{\mathfrak{g},(7,2)}$ and $\left(\xi_{1}, \xi_{3}\right) \notin \mathcal{O}_{\mathfrak{g},(4,2)}, \mathcal{O}_{\mathfrak{g},(4,5)}, \mathcal{O}_{\mathfrak{g},(7,2)} ;\left(\xi_{3}, \xi_{2}\right) \in \mathcal{O}_{\mathfrak{g},(4,3)}, \mathcal{O}_{\mathfrak{g},(4,5)}, \mathcal{O}_{\mathfrak{g},(6,3)}$ and $\left(\xi_{2}, \xi_{3}\right) \notin$ $\mathcal{O}_{\mathfrak{g},(4,3)}, \mathcal{O}_{\mathfrak{g},(4,5)}, \mathcal{O}_{\mathfrak{g},(6,3)}$. But, for every $\nu \in I_{3}^{0}, \mathcal{O}_{\mathfrak{g},(p, q)} \subseteq \mathrm{op}_{\mathfrak{g}, \nu}\left(\mathcal{O}_{\mathfrak{g},(p, q)}\right)$ and $\mathcal{O}_{\mathfrak{g},(p, q)} \in \mathfrak{g}-\nu-\mathrm{F}_{\mathrm{O}}[\emptyset]$ for all $(p, q)=(3,2),(3,6),(4,2),(4,3),(4,5),(6,3),(7,2)$. Thus, for every $\left(\xi_{i}, \xi_{j}\right) \in \mathfrak{g}-\mathrm{F}_{\mathrm{P}}[\sigma>0]$, there exists $\mathcal{O}_{\mathfrak{g},(p, q)} \in \mathfrak{g}-\nu-\mathrm{F}_{\mathrm{O}}[\emptyset]$ such that:

$$
\left[\xi_{i} \in \operatorname{op}_{\mathfrak{g}, \nu}\left(\mathcal{O}_{\mathfrak{g}, p}\right)\right] \wedge\left[\xi_{j} \in \mathrm{op}_{\mathfrak{g}, \nu}\left(\mathcal{O}_{\mathfrak{g}, q}\right)\right] .
$$

This shows that $\mathfrak{T}_{\mathfrak{g}}$ is a $\mathfrak{g}-\mathcal{T}_{\mathfrak{g}}^{(\mathrm{H})}$-space $\mathfrak{g}-\mathfrak{T}_{\mathfrak{g}}^{(\mathrm{H})}=\left(\Omega, \mathfrak{g}-\mathcal{T}_{\mathfrak{g}}^{(\mathrm{H})}\right)$.

Let $\left(\xi_{i}, \xi_{j}\right) \in \mathcal{O}_{\mathfrak{g},(p, q)}$ mean $\xi_{i} \in \mathcal{O}_{\mathfrak{g}, p}, \xi_{j} \in \mathcal{O}_{\mathfrak{g}, q}$, and $\left(\xi_{j}, \xi_{i}\right) \notin \mathcal{O}_{\mathfrak{g},(p, q)}$, where $\mathcal{O}_{\mathfrak{g},(p, q)} \in \mathfrak{g}-\nu-\mathrm{F}_{\mathrm{O}}\left[\mathcal{S}_{\mathfrak{g}} \supset \emptyset\right]$. Then, the following relations are easily verified: $\left(\xi_{1}, \xi_{2}\right) \in \mathcal{O}_{\mathfrak{g},(p, q)}$ and $\left(\xi_{2}, \xi_{1}\right) \notin \mathcal{O}_{\mathfrak{g},(p, q)}$ for all $(p, q)=(2,3),(2,7),(6,3),(6,7)$; $\left(\xi_{1}, \xi_{3}\right) \in \mathcal{O}_{\mathfrak{g},(p, q)}$ and $\left(\xi_{3}, \xi_{1}\right) \notin \mathcal{O}_{\mathfrak{g},(p, q)}$ for all $(p, q)=(2,4),(2,7),(5,4),(5,7)$; $\left(\xi_{2}, \xi_{3}\right) \in \mathcal{O}_{\mathfrak{g},(p, q)}$ and $\left(\xi_{3}, \xi_{1}\right) \notin \mathcal{O}_{\mathfrak{g},(p, q)}$ for all $(p, q)=(3,4),(3,6),(5,4),(5,6)$. But, $\mathcal{O}_{\mathfrak{g},(p, q)} \subseteq \mathrm{op}_{\mathfrak{g}, \nu}\left(\mathcal{O}_{\mathfrak{g},(p, q)}\right)$ for all $(p, q)=(2,3)$, (2,4), (2,7), (3,4), (3,6), $(5,4),(5,6),(5,7),(6,3),(6,7)$. Hence, for every $\left(\xi_{i}, \xi_{j}\right) \in \mathfrak{g}-\mathrm{F}_{\mathrm{P}}[\sigma>0]$, there exists $\mathcal{O}_{\mathfrak{g},(p, q)} \in \mathfrak{g}-\nu-\mathrm{F}_{\mathrm{O}}\left[\mathcal{S}_{\mathfrak{g}} \supset \emptyset\right]$ such that:

$$
\left[\left(\xi_{i}, \xi_{j}\right) \in_{\lambda=p, q} \mathrm{op}_{\mathfrak{g}, \nu}\left(\mathcal{O}_{\mathfrak{g}, \lambda}\right)\right] \wedge\left[\left(\xi_{i}, \xi_{j}\right) \notin_{\lambda=q, p} \mathrm{op}_{\mathfrak{g}, \nu}\left(\mathcal{O}_{\mathfrak{g}, \lambda}\right)\right] .
$$

This shows that $\mathfrak{T}_{\mathfrak{g}}$ is a $\mathfrak{g}-\mathcal{T}_{\mathfrak{g}}^{(\mathrm{F})}$-space $\mathfrak{g}-\mathfrak{T}_{\mathfrak{g}}^{(\mathrm{F})}=\left(\Omega, \mathfrak{g}-\mathcal{T}_{\mathfrak{g}}^{(\mathrm{F})}\right)$. 
Let $\left(\xi_{i}, \xi_{j}\right) \in \mathcal{O}_{\mathfrak{g},(p, q)}$ mean $\xi_{i} \in \mathcal{O}_{\mathfrak{g}, p}$ and $\xi_{j} \notin \mathcal{O}_{\mathfrak{g}, p}$, or $\xi_{i} \notin \mathcal{O}_{\mathfrak{g}, p}$ and $\xi_{j} \in \mathcal{O}_{\mathfrak{g}, q}$, where $\mathcal{O}_{\mathfrak{g},(p, q)} \in \mathfrak{g}-\nu-\mathrm{F}_{\mathrm{O}}\left[\mathcal{S}_{\mathfrak{g}} \supset \emptyset\right]$. Then, the following relations are easily verified: $\left(\xi_{1}, \xi_{2}\right) \in \mathcal{O}_{\mathfrak{g},(2,3)}, \mathcal{O}_{\mathfrak{g},(2,7)}, \mathcal{O}_{\mathfrak{g},(6,3)}, \mathcal{O}_{\mathfrak{g},(6,7)} ;\left(\xi_{1}, \xi_{3}\right) \in \mathcal{O}_{\mathfrak{g},(2,4)}, \mathcal{O}_{\mathfrak{g},(2,7)}, \mathcal{O}_{\mathfrak{g},(5,4)}$, $\mathcal{O}_{\mathfrak{g},(5,7)} ;\left(\xi_{2}, \xi_{3}\right) \in \mathcal{O}_{\mathfrak{g},(3,4)}, \mathcal{O}_{\mathfrak{g},(3,6)}, \mathcal{O}_{\mathfrak{g},(5,4)}, \mathcal{O}_{\mathfrak{g},(5,6)}$. But, $\mathcal{O}_{\mathfrak{g},(p, q)} \subseteq \operatorname{op}_{\mathfrak{g}, \nu}\left(\mathcal{O}_{\mathfrak{g},(p, q)}\right)$ for all $(p, q)=(2,3),(2,4),(2,7),(3,4),(3,6),(5,4),(5,6),(5,7),(6,3),(6,7)$. Hence, for every $\left(\xi_{i}, \xi_{j}\right) \in \mathfrak{g}-\mathrm{F}_{\mathrm{P}}[\sigma>0]$, there exists $\mathcal{O}_{\mathfrak{g},(p, q)} \in \mathfrak{g}-\nu-\mathrm{F}_{\mathrm{O}}\left[\mathcal{S}_{\mathfrak{g}} \supset \emptyset\right]$ such that:

$$
\begin{aligned}
{\left[\left(\xi_{i} \in \mathrm{op}_{\mathfrak{g}, \nu}\left(\mathcal{O}_{\mathfrak{g}, p}\right)\right) \wedge\left(\xi_{j} \notin \mathrm{op}_{\mathfrak{g}, \nu}\left(\mathcal{O}_{\mathfrak{g}, p}\right)\right)\right] } & \vee\left[\left(\xi_{i} \notin \mathrm{op}_{\mathfrak{g}, \nu}\left(\mathcal{O}_{\mathfrak{g}, q}\right)\right)\right. \\
& \left.\wedge\left(\xi_{j} \in \mathrm{op}_{\mathfrak{g}, \nu}\left(\mathcal{O}_{\mathfrak{g}, q}\right)\right)\right] .
\end{aligned}
$$

This shows that $\mathfrak{T}_{\mathfrak{g}}$ is a $\mathfrak{g}-\mathcal{T}_{\mathfrak{g}}^{(\mathrm{K})}$-space $\mathfrak{g}-\mathfrak{T}_{\mathfrak{g}}^{(\mathrm{K})}=\left(\Omega, \mathfrak{g}-\mathcal{T}_{\mathfrak{g}}^{(\mathrm{K})}\right)$.

The elements discussed in the preceding sections can be easily checked from this nice application. In the next section, we provide concluding remarks and future directions of the theory of $\mathfrak{g}-\mathfrak{T}_{\mathfrak{g}}$-separation axioms developed in the earlier sections.

\section{Conclusion}

In this paper, we developed a new theory, called Theory of $\mathfrak{g}$ - $\mathfrak{T}_{\mathfrak{g}}$-Separation Axioms. The theory is based on the Theory of $\mathfrak{g}-\mathfrak{T}_{\mathfrak{g}}$-Sets but not on the Theory of $\mathfrak{g}-\mathfrak{T}_{\mathfrak{g}}$-Maps. In its own rights, the proposed theory has several advantages. The very first advantage is that the theory holds equally well when $\left(\Omega, \mathcal{T}_{\mathfrak{g}}\right)=(\Omega, \mathcal{T})$ and other characteristics adapted on this ground, in which case it might be called Theory of $\mathfrak{g}$-T-Separation Axioms.

Thus, in a $\mathcal{T}_{\mathfrak{g}}$-space the proposed theoretical framework categorises each element of the quintuple sequence $\left\langle\mathfrak{g}-\mathrm{T}_{\mathfrak{g}, \alpha}\right\rangle_{\alpha \in \Lambda}$ as $\mathfrak{g}-\mathrm{T}_{\mathfrak{g}, \alpha}$-axioms of type $\alpha$ and of categories $\nu$, where $(\alpha, \nu) \in \Lambda \times I_{3}^{0}$ and $\Lambda=\{\mathrm{K}, \mathrm{F}, \mathrm{H}, \mathrm{R}, \mathrm{N}\}$ and theorises the concepts in a unified way; in a $\mathcal{T}$-space it categorises each element of the quintuple sequence $\left\langle\mathfrak{g}-\mathrm{T}_{\alpha}\right\rangle_{\alpha \in \Lambda}$ as $\mathfrak{g}-\mathrm{T}_{\alpha}$-axioms of type $\alpha$ and of categories $\nu$, where $(\alpha, \nu) \in \Lambda \times I_{3}^{0}$ and $\Lambda=\{\mathrm{K}, \mathrm{F}, \mathrm{H}, \mathrm{R}, \mathrm{N}\}$ and theorises the concepts in a unified way.

Since the theory of $\mathfrak{g}-\mathfrak{T}_{\mathfrak{g}}$-separation Axioms has been based solely on theory of $\mathfrak{g}-\mathfrak{T}_{\mathfrak{g}}$-sets, as pointed out above, it is an interesting topic for future research either to develop the theory of $\mathfrak{g}-\mathfrak{T}_{\mathfrak{g}}$-separation axioms of mixed categories based on the aforementioned theory or to develop it but based on the theory of $\mathfrak{g}-\mathfrak{T}_{\mathfrak{g}}$-maps. More precisely, either for some pair $(\nu, \mu) \in I_{3}^{0} \times I_{3}^{0}$ such that $\nu \neq \mu$, to develop the theory of $\mathfrak{g}$ - $\mathfrak{T}_{\mathfrak{g}}$-separation axioms based on the theory of $\mathfrak{g}$ - $\mathfrak{T}_{\mathfrak{g}}$-open sets belonging to the class $\left\{\mathcal{O}_{\mathfrak{g}}=\mathcal{O}_{\mathfrak{g}, \nu} \cup \mathcal{O}_{\mathfrak{g}, \mu}:\left(\mathcal{O}_{\mathfrak{g}, \nu}, \mathcal{O}_{\mathfrak{g}, \mu}\right) \in \mathfrak{g}-\nu-\mathrm{O}\left[\mathfrak{T}_{\mathfrak{g}}\right] \times \mathfrak{g}-\mu-\mathrm{O}\left[\mathfrak{T}_{\mathfrak{g}}\right]\right\}$ and the theory of $\mathfrak{g}-\mathfrak{T}_{\mathfrak{g}}$ closed sets belonging to the class $\left\{\mathcal{K}_{\mathfrak{g}}=\mathcal{K}_{\mathfrak{g}, \nu} \cup \mathcal{K}_{\mathfrak{g}, \mu}:\left(\mathcal{K}_{\mathfrak{g}, \nu}, \mathcal{K}_{\mathfrak{g}, \mu}\right) \in \mathfrak{g}-\nu-\mathrm{K}\left[\mathfrak{T}_{\mathfrak{g}}\right] \times\right.$ $\left.\mathfrak{g}-\mu-\mathrm{K}\left[\mathfrak{T}_{\mathfrak{g}}\right]\right\}$ in a $\mathcal{T}_{\mathfrak{g}}$-space $\mathfrak{T}_{\mathfrak{g}}$ or, to develop the theory of $\mathfrak{g}-\mathfrak{T}_{\mathfrak{g}}$-separation axioms based on the theory of $\mathfrak{g}$ - $\mathfrak{T}_{\mathfrak{g}}$-maps, called $\mathfrak{g}$ - $\left(\mathfrak{T}_{\Lambda}, \mathfrak{T}_{\Theta}\right)$-continuous maps, $\mathfrak{g}$ - $\left(\mathfrak{T}_{\Lambda}, \mathfrak{T}_{\Theta}\right)$ irresolute maps and $\mathfrak{g}$ - $\left(\mathfrak{T}_{\Lambda}, \mathfrak{T}_{\Theta}\right)$-homeomorphism maps, where $\Lambda, \Theta \in\{\Omega, \Sigma, \Upsilon\}$, between any two of such $\mathcal{T}_{\mathfrak{g}}$-spaces $\mathfrak{T}_{\mathfrak{g}, \Omega}, \mathfrak{T}_{\mathfrak{g}, \Sigma}$, and $\mathfrak{T}_{\mathfrak{g}, \Upsilon}$. Such two theories are what we thought would certainly be worth considering, and the discussion of this paper ends here.

\section{Acknowledgments}

The authors would like to express their sincere thanks to Prof. Endre Makai, Jr. (Professor Emeritus of the Mathematical Institute of the Hungarian Academy of Sciences) for his valuable suggestions. 


\section{Funding}

This research received no specific grant from any funding agency in the public, commercial, or not-for-profit sectors.

The Declaration of Conflict of Interest/ Common Interest

The author(s) declared that no conflict of interest or common interest

\section{The Declaration of Ethics Committee Approval}

This study does not be necessary ethical committee permission or any special permission.

\section{The Declaration of Research and Publication Ethics}

The authors declared that they comply with the scientific, ethical, and citation rules of Journal of Universal Mathematics in all processes of the study and that they do not make any falsification on the data collected. Besides, the authors declared that Journal of Universal Mathematics and its editorial board have no responsibility for any ethical violations that may be encountered and this study has not been evaluated in any academic publication environment other than Journal of Universal Mathematics.

\section{REFERENCES}

[1] W. K. Min, Remarks on Separation Axioms on Generalized Topological Spaces, Journal of the Chungcheong Mathematical Society, vol. 23, N. 2, pp. 293-298 (2010).

[2] D. Narasimhan, An Overview of Separation Axioms in Recent Research, International Journal of Pure and Applied Mathematics, vol. 76, N. 4, pp. 529-548 (2012). ISSN: $1311-8080$

[3] B. Roy and R. Sen and T. Noiri, Separation Axioms on Topological Spaces: A Unified Version, European Journal of Pure and Applied Mathematics, vol. 6, N. 1, pp. 44-52 (2013). ISSN 1307-5543

[4] C. Carlos and R. Namegalesh and R. Ennis, Separation Axiom on Enlargements of Generalized Topologies, Revista Integración, vol. 32, N. 1, pp. 19-26 (2014).

[5] A. Danabalan and C. Santhi, A Class of Separation Axioms in Generalized Topology, Mathematical Journal of Interdisciplinary Sciences, vol. 4, N. 2, pp. 151-159 (2016).

[6] J. Dontchev, On Some Separation Axioms Associated with the $\alpha$-Topology, Mem. Fac. Sci. Kochi Univ. Ser. A, Math., vol. 18, pp. 31-35 (1997).

[7] S. P. Missier and A. Robert, Higher Separation Axioms via Semi*-Open Sets, Int. Journal of Engineering and Science, vol. 4, N. 6, pp. 37-45 (2014).

[8] T. C. K. Raman and V. Kumari and M. K. Sharma, $\alpha$-Generalized and $\alpha^{*}$-Separation Axioms for Topological Spaces, IOSR Journal of Mathematics, vol. 10, N. 3, pp. 32-36 (2014).

[9] F. G. Arenas and J. Dontchev and M. L. Puertas, Unification Approach to the Separation Axioms Between $T_{0}$ and Completely Hausdorff, Acta Math. Hungar., vol. 86, N. 1-2, pp. 75-82 (2000).

[10] M. M. Deza and E. Deza, Encyclopedia of Distances, Discrete Mathematics, Springer-Verlag, Berlin, (2009). 
[11] K. El-Saady and F. Al-Nabbat, Separation Axioms in Generalized Base Spaces, British Journal of Mathematics and Computer Science, vol. 17, N. 2, pp. 1-8 (2016).

[12] R. Ennis and C. Carlos and S. José, $\gamma-(\alpha, \beta)$-Semi Sets and New Generalized Separation Axioms, Bull. Malays. Sci. Soc. (2), vol. 30, N. 1, pp. 13-21 (2007).

[13] A. Keskin and T. Noiri, Higher Separation Axioms via Semi*-Open Sets, Bulletin of the Iranian Mathematical Society, vol. 35, N. 1, pp. 179-198 (2009).

[14] M. I. Khodabocus and N. -U. -H. Sookia, heory of Generalized Sets in Generalized Topological Spaces, Journal of New Theory, vol. 36, N. 2021, pp. 18-38 (2021).

[15] V. Pankajam and D. Sivaraj, Some Separation Axioms in Generalized Topological Spaces, Bol. Soc. Paran. Mat., vol. 31, N. 1, pp. 29-42 (2013).

[16] D. Pratulananda and A. R. Mamun, $g^{*}$-Closed Sets and a New Separation Axiom in Alexandroff Spaces, Archivum Mathematicum (BRNO), vol. 39, pp. 299-307 (2003).

[17] L. A. Steen and J. A. Jr. Seebach, Counterexamples in Topology, Springer, New York, (1978).

[18] Á. Császár, Separations Axioms for Generalized Topologies, Acta Math. Hungar., vol. 104, N. 1-2, pp. 63-69 (1998).

[19] M. S. Sarsak, New Separation Axioms in Generalized Topological Spaces, Acta Math. Hungar., vol. 132, N. 3, pp. 244-252 (2011).

[20] L. L. L. Lusanta and H. M. Rara, Generalized Star $\alpha-b-$ Separation Axioms in Bigeneralized Topological Spaces, Applied Mathematical Sciences, vol. 9, N. 75, pp. 3725-3737 (2015).

[21] M. V. Mielke, Separation Axioms and Geometric Realizations, Indian J. Pure Appl. Math., vol. 25, N. 7 , pp. 711-722 (1994).

[22] A. D. Ray and R. Bhowmick, Separation Axioms on Bi-Generalized Topological Spaces, Journal of the Chungcheong Mathematical Society, vol. 27, N. 3, pp. 363-379 (year).

[23] J. Thomas and S. J. John, Soft Generalized Separation Axioms in Soft Generalized Topological Spaces, International Journal of Scientific and Engineering Research, vol. 6, N. 3, pp. 969-974 (year).

[24] Á. Császár, Remarks on Quasi-Topologies, Acta Math. Hungar., vol. 119, N. 1-2, pp. 197-200 (2008).

[25] Á. Császár, Generalized Open Sets in Generalized Topologies, Acta Math. Hungar., vol. 106, N. 1-2, pp. 53-66 (2005).

[26] V. Pavlović and A. S. Cvetković, On Generalized Topologies arising from Mappings, Vesnik, vol. 38, N. 3, pp. 553-565 (year).

[27] S. Bayhan and A. Kanibir and I. L. Reilly, On Functions between Generalized Topological Spaces, Appl. Gen. Topol., vol. 14, N. 2, pp. 195-203 (2013).

[28] C. Boonpok, On Generalized Continuous Maps in Čech Closure Spaces, General Mathematics, vol. 19, N. 3, pp. 3-10 (2011).

[29] A. S. Mashhour and A. A. Allam and F. S. Mahmoud and F. H. Khedr, On Supratopological Spaces, Indian J. Pure. Appl. Math., vol. 14, N. 4, pp. 502-510 (1983). 
[30] M. I. Khodabocus, A Generalized Topological Spaces endowed with Generalized Topologies, PhD Thesis, University of Mauritius, (2020).

(M. I. Khodabocus) University of Mauritius, Faculty of Science, Department of MathEMATICS, RÉDUit 80837, MAURITIUS

Current address: Université des Mascareignes, Faculty of Sustainable Development and Engineering, Department of Emerging Technologies, Rose Hill Campus, MAURITIUS

Email address: ikhodabocus@udm.ac.mu

(N. Ul-. H. Sookia) University of Mauritius, Faculty of Science, Department of MathEMATICS, RÉDUit 80837, MAURITIUS

Email address: sookian@uom.ac.mu 